\title{
Pesquisas sob amostragem informativa utilizando o FBST
}

\author{
Daniel Mendes Azeredo \\ TESE APRESENTADA \\ $\mathrm{AO}$ \\ Instituto DE MATEMÁtica E Estatística \\ DA \\ Universidade de SÃo Paulo \\ PARA \\ OBTENÇÃO DO TÍTULO \\ $\mathrm{DE}$ \\ Doutor EM CiÊnCIAS \\ Área de Concentração: Matemática Aplicada \\ Orientador: Prof. Dr. Julio Michael Stern
}

Durante a elaboração deste trabalho o autor recebeu auxílio financeiro da CNPq

São Paulo, maio de 2013 


\section{Pesquisas sob amostragem informativa utilizando FBST}

Esta versão da tese contém as correções e alterações sugeridas pela Comissão Julgadora durante a defesa da versão original do trabalho, realizada em 28/05/2013. Uma cópia da versão original está disponível no

Instituto de Matemática e Estatística da Universidade de São Paulo.

Banca Examinadora:

- Prof. Dr. Júlio Michael Stern (orientador) - IME USP

- Prof. Dr. Carlos Alberto de Bragança Pereira - IME USP

- Prof. Dr. Marcelo de Souza Lauretto - EACH USP

- Prof. Dr. Fabio Nakano - EACH USP

- Profa. Dra. Laura Leticia Ramos Rifo - UNICAMP 
Saber viver

Não sei... se a vida é curta ou longa para nós, mas sei que nada do que vivemos tem sentido, se não tocarmos o coração das pessoas.

Muitas vezes basta ser:

colo que acolhe, braço que envolve, palavra que conforta, silêncio que respeita, alegria que contagia, lágrima que corre, olhar que acaricia, desejo que sacia, amor que promove. E isso não é coisa de outro mundo, é o que dá sentido à vida. É o que faz com que ela não seja nem curta, nem longa demais, mas que seja intensa, verdadeira, pura... Enquanto durar.

Cora Coralina [1889-1985] 



\section{Agradecimentos}

Antes de mais nada, agradeço à toda minha família pelo carinho, dedicação e amor que sempre me ofereceram. Agradeço especialmente à minha mãe, uma mulher extraordinária, uma incansável trabalhadora e um exemplo de vida. Minha gratidão especial também ao meu pai, aos meus irmãos, às minhas queridas avós (Maria e Cleusa), à minha tia Ivete e à minha amorosa namorada Fabíola, por toda atenção, carinho e incentivo.

Agradeço de maneira muito especial ao meu dedicado orientador, professor Julio Michael Stern, por toda a paciência, dedicação, incentivo e incalculável ensinamento durante nossas reuniões ao longo desses mais de quatro anos de trabalho juntos.

Minha gratidão também aos incomparáveis docentes e funcionários do Instituto de Matemática e Estatística da Universidade de São Paulo, por toda a dedicação, atenção, apoio e pelos ensinamentos que me acompanharão por toda minha vida. Em especial agradeço ao professor Alexandre Megiorin Roma pela orientação no programa de mestrado e no primeiro semestre do doutorado; aos professores Marcelo Lauretto e Fabio Nakano pelas significativas considerações durante o exame de qualificação e aos professores Joyce da Silva Bevilacqua e Claudio Hirofume Asano pela oportunidade de trabalharmos juntos e pelos inúmeros ensinamentos na arte de ensinar Cálculo Numérico.

Pela generosa ajuda nos momentos de dúvida e dificuldade durante a elaboração desse trabalho, gostaria de agradecer aos amigos Renato Viana Boy, Ivan Mamede Carlos, Nilton Manuel Évora do Rosário, Marlon Wisner Valgas, Diane Rizzotto Rossetto, Flavia Maria Ré, Pedro da Silva Peixoto, Wellington Carlos de Jesus, Carla Reis, Nelson Leonardo Navarrete, Eduardo Oda, Ricardo de Lima Ribeiro, Marcelo Caetano, Francisco Sokol, 
Marcello Souza de Oliveira, Nils Mirã, Larissa Sartori, Marline Silva, Silvio Rodrigues de Faria Junior e Camila Bertini Martins.

Meus sinceros agradecimentos também ao seu Lauro e à Dona Sueli, que juntamente com toda sua família me acolheram como um filho em São Paulo, tornando possível os nossos memoráveis almoços de domingo em família.

Não tenho como expressar minha gratidão para com todo o corpo docente e os funcionários do Instituto de Matemática e Estatística da Universidade Federal de Goiás (UFG), por me tolerarem pacientemente durante os quatro anos da graduação e por toda a dedicação na arte de ensinar Matemática e formar professores e pesquisadores nessa área. Em especial aos professores e amigos Geci José Pereira da Silva e Rosely Maria Barbosa Goes.

Não poderia deixar de agradecer também a todos os amigos que tive o imenso prazer de conhecer e conviver na UFG, que prefiro não tentar colocar os nomes deles aqui porque tenho certeza que vou esquecer de citar alguém, por toda a ajuda, apoio e torcida. Entre eles, agradeço imensamente aos onze amigos que terminaram o curso de bacharelado em Matemática comigo, pelo companherismo e amizade durante os momentos difíceis.

E, por fim, seria um relapso muito grande da minha parte se não agradecesse a Conselho Nacional de Pesquisa e Desenvolvimento - CNPq pela bolsa de doutorado que possibilitou a elaboração do presente trabalho.

A todos, o meu muito obrigado. 


\section{Resumo}

Azeredo, D. M. Pesquisas sob amostragem informativa utilizando o FBST. 2013. 107 f. Tese (Doutorado). Instituto de Matemática e Estatística. Universidade de São Paulo, São Paulo, 2013.

Pfeffermann, Krieger e Rinott [28] apresentaram uma metodologia para modelar processos de amostragem que pode ser utilizada para avaliar se este processo de amostragem é informativo. Neste cenário, as probabilidades de seleção da amostra são aproximadas por uma função polinomial dependendo das variáveis resposta e concomitantes. Nesta abordagem, nossa principal proposta é investigar a aplicação do teste de significância FBST ( Full Bayesian Significance Test), apresentado por Pereira e Stern [24], como uma ferramenta para testar a ignorabilidade amostral, isto é, para avaliar uma relação de significância entre as probabilidades de seleção da amostra e a variável resposta. A performance desta modelagem estatística é testada com alguns experimentos computacionais.

Palavras-chaves: Amostragem informativa; Distribuição amostral; Amostragem PPT; Ignorabilidade amostral; FBST. 



\section{Abstract}

Azeredo, D. M. Surveys under informative sampling using the FBST. 2013. 107 f. Tese (Doutorado). Instituto de Matemática e Estatística. Universidade de São Paulo, São Paulo, 2013.

Pfeffermann, Krieger and Rinott [28] introduced a framework for modeling sampling processes that can be used to assess if a sampling process is informative. In this setting, sample selection probabilities are approximated by a polynomial function depending on outcome and auxiliary variables. Within this framework, our main purpose is to investigate the application of the Full Bayesian Significance Test (FBST), introduced by Pereira and Stern [24], as a tool for testing sampling ignorability, that is, to detect a significant relation between the sample selection probabilities and the outcome variable. The performance of this statistical modelling framework is tested with some simulation experiments.

Keywords: Design variables; Informative sampling; Sample distribution; PPS sampling; Sampling ignorability; FBST - Full Bayesian Significance Test. 



\section{Sumário}

Lista de figuras $\quad$ xiii

Lista de tabelas $\quad$ Xv

1 Introdução 1

2 Amostragem informativa $\quad 15$

2.1 Projetos amostrais informativos e ignoráveis . . . . . . . . . . . . 16

2.2 Mecanismos de não-resposta informativos e ignoráveis . . . . . . . . . . . . 19

2.3 Distribuição marginal amostral . . . . . . . . . . . . . . . . . . . . . 22

2.4 Distribuição marginal respondente . . . . . . . . . . . . . . . . . . . 25

2.5 Relações gerais de invariância . . . . . . . . . . . . . . . . . . 27

2.6 Distribuições amostrais em projetos amostrais gerais . . . . . . . . . . . . . 28

2.6.1 Seleção com probabilidade proporcional ao tamanho . . . . . . . . . 29

2.6.2 Seleção com amostragem estratificada . . . . . . . . . . . . . . . . 31

3 FBST $\quad 35$

3.1 Definição intuitiva do FBST . . . . . . . . . . . . . . . . 36

3.2 Definição invariante do FBST . . . . . . . . . . . . . . . . . . . . . . 38

3.3 Função de perda para o FBST . . . . . . . . . . . . . . . . . . . 40

3.4 Consistência do FBST . . . . . . . . . . . . . . . . . . . 42 
3.5 Aspectos computacionais do FBST . . . . . . . . . . . . . . . . . 44

3.5.1 Otimização . . . . . . . . . . . . . . . . . . . 44

3.5.2 Integração . . . . . . . . . . . . . . . . . . . . . . . 45

4 Simulações numéricas $\quad 49$

4.1 Distribuição das probabilidades de seleção da amostra conhecida . . . . . . 50

4.2 Distribuição das probabilidades de seleção da amostra desconhecida . . . . 52

4.3 Seleção da amostra . . . . . . . . . . . . . . . . . . . . . 54

4.4 Implementação das simulações . . . . . . . . . . . . . . . . . . . . . . . 55

4.5 Resultados das simulações . . . . . . . . . . . . . . . . . . . 57

5 Considerações finais $\quad 65$

Referências bibliográficas $\quad 72$

$\begin{array}{ll}\text { Índice } & 73\end{array}$

$\begin{array}{ll}\text { A Artigo resultante da tese } & 75\end{array}$ 


\section{Lista de Figuras}

1.1 Exemplo de amostragem informativa. . . . . . . . . . . . . . . 5

4.1 Polinômios aproximadores. . . . . . . . . . . . . . . . . . . 63 


\section{Lista de Tabelas}

4.1 Viés relativo porcentual (amostragem aleatória simples com substituição). . 58

4.2 Viés relativo porcentual (amostragem proporcional ao tamanho com substituição) . . . . . . . . . . . . . . . . . . . 5 58

4.3 Viés relativo porcentual (amostragem sucessiva) . . . . . . . . . . . . 59

4.4 Viés relativo porcentual (método de Sampford) . . . . . . . . . . . . . 59

4.5 Viés relativo porcentual (amostragem rejeitiva) . . . . . . . . . . . 60

4.6 Viés relativo porcentual (amostragem sistemática) . . . . . . . . . . 60

4.7 Viés relativo porcentual: casos aceitos $\times$ casos rejeitados (amostragem proporcional ao tamanho com substituição). . . . . . . . . . . . . . . . . 61

4.8 Viés relativo porcentual: casos aceitos $\times$ casos rejeitados (amostragem sucessiva). . . . . . . . . . . . . . . . . . 6 6 61

4.9 Viés relativo porcentual: casos aceitos $\times$ casos rejeitados (método de Samp-

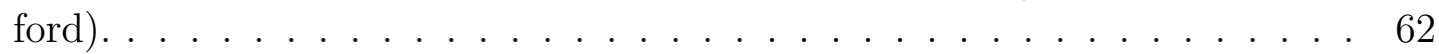

4.10 Viés relativo porcentual: casos aceitos $\times$ casos rejeitados (amostragem rejeitiva). . . . . . . . . . . . . . . . 62

4.11 Viés relativo porcentual: casos aceitos $\times$ casos rejeitados (amostragem sistemática). . . . . . . . . . . . . . . . 62 


\section{CAPÍtulo 1}

\section{Introdução}

Pesquisas por amostragem são frequentemente realizadas em áreas relacionadas a agricultura, economia, educação, política, saúde e vários outros tópicos de interesse. Organizações governamentais nacionais e internacionais e agências privadas coletam informações por meio dessas pesquisas em questões como produção industrial e agrícola, taxas de desemprego, popularidade de políticos que ocupam cargos públicos elegíveis, eficácia de medicamentos no tratamento de doenças, relação entre incidência de doenças e fatores de risco, e intenção de votos durante o período eleitoral.

As informações dessas pesquisas são essenciais, por exemplo, para planejamento econômico e agrícola, desenvolvimento de procedimentos médicos e educacionais e melhoria de serviços públicos. O objetivo principal dessas pesquisas é estimar características da população estudada utilizando as informações coletadas em uma amostra.

A grande vantagem em utilizar pesquisas por amostragem é que, quando realizadas com um projeto amostral representativo, elas podem fornecer em pouco tempo, com um baixo custo e boa precisão, informações importantes para problemas práticos. Por projeto amostral entende-se o procedimento utilizado para selecionar as unidades de uma população que serão incluídas na amostra e o mesmo é representativo quando a amostra selecionada tem as mesmas propriedades que a população de interesse.

Diante da dificuldade em avaliar se um projeto amostral é representativo sem conhecer as informações da população de interesse, é preferível trabalhar com o conceito de projeto amostral probabilístico, que são os procedimentos onde cada possível amostra tem uma probabilidade conhecida de ocorrer. O caso mais simples de amostra probabilística 
ocorre quando os elementos da população são sorteados sequencialmente com mesma probabilidade de pertecerem a amostra. Neste caso diz-se que a amostra foi sorteada por Amostragem Aleatória Simples. A escolha da amostra com probabilidade igual em todas as unidades da população é um procedimento que simplifica muito o desenvolvimento das propriedades associadas ao projeto amostral.

Entretanto, em algumas situações é conveniente sortear a amostra com probabilidades de seleção desiguais. Uma forma de projeto amostral com probabilidades desiguais amplamente utilizado é Amostragem Aleatória Estratificada que consiste na divisão da população em subgrupos, chamados estratos, segundo alguma característica conhecida da população em estudo e, dentro de cada um desses estratos são selecionadas amostras com tamanhos convenientes. O resultado da pesquisa é mais eficaz quanto mais homogêneos forem os estratos escolhidos. A grande vantagem em utilizar esse método é o aumento da eficiência e precisão das estimativas e a desvantagem é que para isso é necessário conhecer os resultados de uma pesquisa anterior com informações das características que se deseja pesquisar na população de interesse.

Quando essas informações da população não estão disponíveis ou estão desatualizadas e o custo de atualizá-las é muito alto, a amostragem pode ser facilitada dividindo a população em grupos, denominados conglomerados, segundo algum critério conveniente. Geralmente, os conglomerados são escolhidos como regiões geográficas. Esta forma de projeto amostral com probabilidades de seleção desiguais, denominada Amostragem Aleatória por Conglomerados, consiste na divisão da população em conglomerados e, no sorteio dos conglomerados para a amostra. Posteriormente, dentro de cada conglomerado escolhido, pode ser feita a coleta das informações desejadas em todas as unidades da população ou pode ser realizado um novo sorteio para determinar as unidades da população pertencentes aos conglomerados que responderão à pesquisa.

Escolher um projeto amostral adequado é uma das partes mais importantes em pesquisas por amostragem, pois o planejamento e operação do processo de amostragem costumam ser a parte de maior custo da pesquisa e, por isso, o projeto amostral deve ser realizado com o objetivo de minimizar este custo condicionado aos limites aceitáveis de incerteza das informações desejadas.

Depois de planejar o projeto amostral, um problema muito comum que pode ocorrer durante a coleta de dados de uma pesquisa por amostragem é uma falha na obtenção 
de respostas válidas das unidades da amostra. Quando há uma algum tipo de falha na resposta de um ou mais itens da pesquisa, esse tipo de dado faltante é chamado nãoresposta ao item. Basicamente, existem dois tipos de não-resposta ao item: completa e parcial.

A não-resposta completa ocorre quando todas as variáveis da pesquisa estão faltando ou não há informação útil suficiente disponível. Neste caso a unidade amostrada é identificada como completamente não-respondente, mesmo que alguma informação tenha sido coletada, pois ela foi considerada insuficiente. Isto ocorre geralmente quando uma unidade da amostra se recusa a responder a pesquisa ou encerra prematuramente a entrevista. A não-resposta parcial ocorre quando algumas, mas não todas, as variáveis da pesquisa tem informações faltantes. Isto é comum em casos que a unidade da amostra se recusa a responder alguma questão considerada delicada ou não sabe a resposta de alguns itens.

Um aspecto importante a ser considerado nos processos de amostragem é como gerenciar e reduzir a proporção de não-respostas, pois elas podem aumentar o viés e reduzir a precisão das estimativas obtidas. Essa proporção de não-respostas tende a ser menor quando o assunto da pesquisa está relacionado com reformas educacionais, mudanças nos impostos, melhorias regionais, questões políticas e eleitorais e tópicos similares. As altas proporções de não-respostas geralmente ocorrem em pesquisas relacionadas às características pessoais, como rendimentos, patrimônios, doenças pré-existentes e questões relacionadas à sexualidade.

Neste sentido, o objetivo principal ao considerar os casos de não-resposta ao item (completa ou parcial) é reduzir o viés provocado pela não-resposta, que ocorre se as probabilidades de não-resposta de um item estiver relacionada com a variável resposta.

Para compreendermos por que modelar dados de pesquisa pode ser complicado, suponhamos que o modelo assumido para ajustar os valores populacionais (distribuição populacional) é especificado precisamente. Se no processo de amostragem, as unidades da amostra forem sorteadas por amostragem aleatória simples e todas essas unidades amostradas responderem, então o modelo populacional pode ser considerado adequado também para dados provenientes da amostra e pode ser ajustado utilizando qualquer método estatístico apropriado para o problema, como métodos de mínimos quadrados, de máxima verossimilhança ou bayesianos. 
Entretanto, a maioria dessas pesquisas utiliza mecanismos complexos de seleção da amostra, nos quais as unidades da população são sorteadas com probabilidades de escolha distintas no processo de amostragem podendo, em alguns casos, acontecer dessas probabilidades de seleção ou mesmo as probabilidades de não-resposta ao item estarem associadas à variável resposta. Consequentemente, o modelo adequado para os dados coletados no processo de amostragem pode ser muito diferente do modelo válido para a população.

Apesar disso, muitas vezes a inferência é feita ignorando o mecanismo pelo qual a amostra foi escolhida e as não-respostas foram obtidas e, por consequência, as unidades da amostra são analisadas como se fossem independentes e identicamente distribuídas e o mecanismo de não-respostas é considerado aleatório. Isso nem sempre é verdade, pois tanto o processo de seleção da amostra quanto o mecanismo de não-respostas podem ser informativos, isto é, as probabilidades de seleção da amostra ou as probabilidades de não-resposta nas unidades da amostra podem estar correlacionadas com a variável resposta do modelo. Nesse caso, o modelo válido para os dados obtidos é diferente do modelo populacional. Quando o projeto amostral e o mecanismo de não-respostas não são informativos, diz-se que os mesmos são ignoráveis.

Dessa maneira, define-se o modelo amostral (ou distribuição amostral) como sendo o modelo válido para os dados coletados no processo de amostragem sem considerar o mecanismo de não-respostas. Quando a amostragem é informativa, este modelo é diferente do modelo populacional e deve ser utilizado no processo de inferência. Analogamente, define-se modelo respondente (ou distribuição respondente) como sendo o modelo válido para os dados amostrais considerando o mecanismo de não-respostas. Quando o mecanismo de não-respostas é informativo, este modelo é diferente dos modelos populacional e amostral, e deve ser considerado para estimação dos parâmetros do modelo populacional. Claramente, quando nem o processo de amostragem e nem o mecanismo de não-respostas são informativos, as distribuições populacional, amostral e respondente coincidem. Outrossim, quando a amostragem não é informativa, os modelos populacional e amostral coincidem não sendo sempre verdade o mesmo para o modelo respondente.

Para exemplificar a importância do processo de amostragem, foi realizada uma simulação computacional considerando uma população com $N=500$ indivíduos tal que a variável resposta $y_{i}$ tem distribuição normal com média $0,5+1,5 x_{i}$ e variância 1 com $x_{i}$ tendo distribuição uniforme no intervalo $[0,1]$ para cada $i=1, \ldots, N$. Dessa população 
foram sorteadas três amostras, cada uma de tamanho $n=100$ : na primeira amostra todos os indivíduos da população foram sorteados com a mesma probabilidade de seleção e todos as respostas estavam completas; na segunda amostra os indivíduos foram selecionados com probabilidade proporcional a $\exp \left(5 y_{i}\right)$; e na terceira amostra os elementos da amostra foram sorteados com probabilidade de proporcional a $\exp \left(-5 y_{i}\right)$. Com isso, a segunda e a terceira seleção da amostra são informativas, pois as probabilidades de seleção da amostra estão associadas com a variável resposta e portanto as distribuições populacional e amostral são diferentes. A Figura 1.1 mostra cada uma das amostras selecionadas e a reta representado a distribuição utilizada para gerar os dados populacionais.

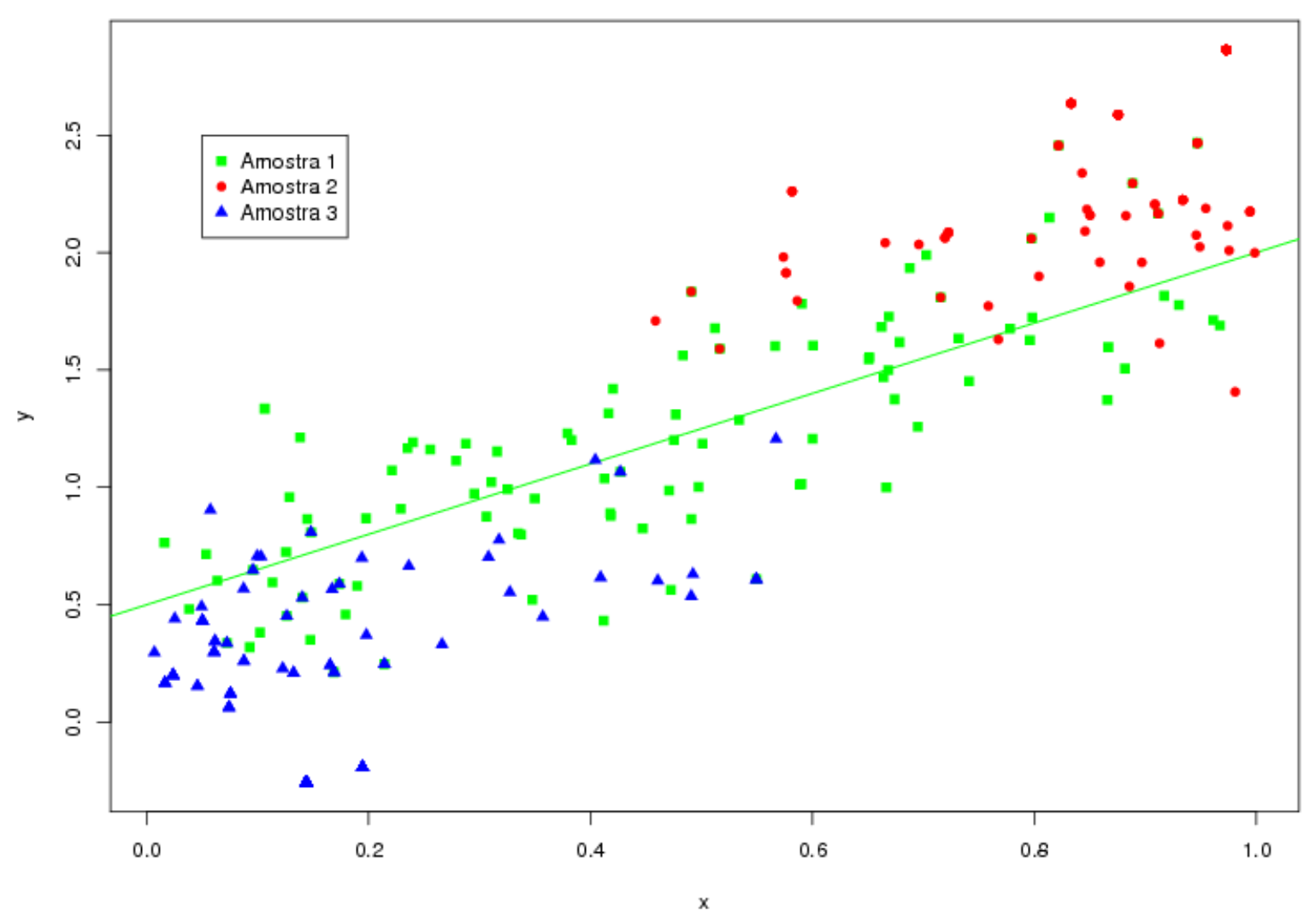

Figura 1.1: Exemplo de amostragem informativa.

Por conseguinte, realizar inferência estatística sem considerar o mecanismo de seleção da amostra e o mecanismo de não-resposta quando pelo menos um deles é informativo pode 
produzir estimativas viciadas para os parâmetros de interesse, resultando em considerações distorcidas do fenômeno em estudo.

Para superar essa dificuldade em trabalhar com os procedimentos padrão de inferência quando a amostragem é informativa, Pfeffermann, Krieger e Rinott [28] propuseram uma expressão para calcular a distribuição amostral definindo-a em função da distribuição populacional e dos valores esperados para as probabilidades de inclusão na amostra. Posteriormente, Pfeffermann e Sikov [33] estenderam esta metodologia para os casos em que o mecanismo de não-resposta também é informativo, definindo uma expressão para o modelo respondente em função da distribuição populacional, das probabilidades de inclusão na amostra e das probabilidades de não-resposta. A principal vantagem em fazer inferência utilizando essas metodologias é que isso permite a utilização dos procedimentos básicos de inferência estatística.

Desenhos amostrais complexos têm sido muito utilizados em pesquisas sociais e médicas com estrutura hierárquica para fornecer estimativas não só da população de interesse, mas também de uma ampla variedade de subpopulações (domínios). Domínios podem ser definidos por regiões geográficas, grupos sócio-demográficos ou outras subpopulações. Exemplos de domínios geográficos (áreas) incluem países, estados, municípios, distritos escolares, regiões metropolitanas e regiões de serviço de saúde. Por outro lado, domínios sócio-demográficos podem se referir a grupos específicos divididos por idade, raça ou gênero em uma área geográfica específica.

Pfeffermann, Moura e Silva [31], por exemplo, analisaram um estudo educacional de proficiência de alunos que utiliza um projeto amostral informativo hierárquico com dois níveis, com escolas no primeiro nível de amostragem e alunos no segundo nível. A amostragem das escolas foi realizada com probabilidade proporcional ao tamanho das escolas (quantidade de alunos), propiciando que as escolas com maior número de alunos tivessem maior probabilidade de pertencer à amostra e dificultando que escolas rurais (maior custo de amostragem e menor quantidade de alunos) estivessem na amostra. Entretanto, como nesse estudo o tamanho das escolas estava relacionado com a proficiência dos alunos (variável resposta do modelo), a amostragem é informativa e as escolas da amostra não representam apropriadamente as escolas da comunidade analisada. Depois de escolhidas as escolas da amostra, a amostragem dos alunos das escolas sorteadas foi realizada por amostragem estratificada desproporcional. Contudo, observou-se que os estratos escolhi- 
dos também estavam associados com a proficiência dos alunos, ocorrendo novamente um caso de amostragem informativa no segundo nível hierárquico do modelo.

Em um trabalho mais recente, Pfeffermann e Sikov [33] analisaram uma pesquisa de despesas domiciliares baseadas em características socio-demográficas considerando probabilidades iguais de seleção da amostra (modelo populacional igual ao modelo amostral) e definindo como domicílios respondentes aqueles que responderam ao questionário na primeira tentativa de entrevista e como não-respondentes os domicílios que não responderam na primeira tentativa de entrevista, mas responderam em uma das tentativas seguintes. Nessa pesquisa observou-se que as probabilidades de não-resposta estavam associadas com a variável resposta do modelo e para modelar essa probabilidade de não-resposta, dada a variável resposta e as covariáveis, foi considerada uma distribuição logística. Como os dados dos domicílios respondentes e não-respondentes são conhecidos, foi possível comparar as estimativas obtidas para os parâmetros do modelo ajustando todos os dados coletados para a amostra (respondentes e não-respondentes) utilizando o modelo populacional com as estimativas obtidas ajustando somente os dados amostrais respondentes utilizando a distribuição respondente. Com isso, observou-se que as estimativas obtidas para os parâmetros utilizando o modelo respondente nos casos de não-resposta informativa e as estimativas obtidas utilizando o modelo populacional com todos os dados da amostra tiveram precisão semelhante.

Além disso, amostragem informativa tem sido considerada também em modelos hierárquicos utilizados em problemas de estimação em pequenas áreas (Small Area Estimation). O termo pequenas áreas denota qualquer domínio em que estimativas utilizando somente dados amostrais desse domínio específico não podem ser realizadas com a precisão adequada. Mesmo quando uma pesquisa tem tamanho amostral suficientemente grande para fazer estimativas para toda a população desse domínio, esse tamanho amostral pode não ser grande o suficiente para fazer estimativas para subgrupos desse domínio e raramente é possível ter uma amostra com tamanho suficientemente grande para garantir boas estimativas para cada subgrupo considerando somente dados desse subgrupo.

Após a estimação das propriedades dos parâmetros do modelo, surge o controverso problema de inferência estatística de teste de significância para hipóteses precisas. Segundo Kempthorne [15] e Cox [8], testes de significância são procedimentos para medir a consistência dos dados com alguma hipótese precisa. A idéia básica de significância é uma 
ordenação para o espaço amostral de acordo com o aumento da consistência dos dados em relação à hipótese.

Uma medida de evidência completamente bayesiana para testes de significância de hipóteses precisas, cujo teste de significância foi intitulado FBST (Full Bayesian Significance Test), foi proposta por Pereira e Stern [24] e consiste na análise de conjuntos de credibilidade. Por completamente bayesiano entende-se que é necessário conhecer somente o espaço paramétrico, representado pelo distribuição a posteriori, sem a necessidade de nenhum artifício como supor probabilidade positiva para hipóteses precisas.

Deve-se enfatizar que, no caso geral do FBST, a distribuição a posteriori é suficiente para calcular o valor de evidência, sem qualquer complicação devido à dimensionalidade dos parâmetros ou do espaço amostral. Além disso, sob algumas condições de regularidade, esse cálculo é invariante sob transformações no sistema de coordenadas no espaço de parâmetros ou reparametrizações da hipótese a ser testada, isso é importante pois alguns procedimentos estatísticos não satisfazem essa propriedade. Pereira e Lindley [23], por exemplo, discutem o problema de teste de homogeneidade para proporções mostrando como diferentes parametrizações da hipótese podem produzir respostas diferentes.

Além disso, o FBST é intuitivo, tem uma fácil interpretação geométrica, pode ser facilmente implementado utilizando modernas técnicas de integração e otimização numérica, e é um procedimento exato, isto é, não utiliza no cálculo do valor de evidência qualquer aproximação assintótica.

Um outro importante aspecto do FBST é que esse teste permite a incorporação de experiência prévia ou opiniões de especialistas por meio da distribuição a priori. Porém, se o objetivo for medir a consistência dos dados com a hipótese nula sem envolver a distribuição a priori, a distribuição a posteriori pode ser substituída pela função de verossimilhança desde que a integral dessa função seja limitada.

O FBST utiliza uma probabilidade a posteriori bem definida para qualquer subconjunto do espaço de parâmetros e não viola o Príncipio da Verossimilhança, isto é, toda a informação obtida a partir das observações pode ser representada pela função de verossimilhança.

Como foi mostrado por Madruga, Esteves e Wechsler [19], o FBST é consistente sob o ponto de vista da teoria bayesiana da decisão de Rubin [37], pois existe uma função de 
perda específica que avalia aspectos da teoria da decisão para o FBST. Apesar do FBST satisfazer os aspectos da teoria de decisão, ele foi originalmente definido baseado somente no príncipio jurídico Onus Probandi, também conhecido como Presunção de Inocência, que estabelece que ninguém será considerado culpado até o trânsito em julgado de sentença penal condenatória.

No presente trabalho, pretende-se discutir maneiras coerentes de tratar as seguintes questões que surgem na inferência de dados coletados por amostragem:

1. Supondo que o modelo populacional é especificado precisamente e dado um conjunto de dados obtidos por amostragem, o processo de coleta da amostra é informativo? Como avaliar isso?

2. Se a amostragem for informativa, como obter modelos que melhoram a estimativa dos parâmetros do modelo?

3. Qual desses modelos melhor ajusta os dados, de acordo com algum critério dado?

Para discutir essas questões utilizou-se a metodologia proposta por Pfeffermann, Krieger e Rinott [28] para inferência de dados sob amostragem informativa, definindo-se a distribuição válida para a amostra selecionada (distribuição amostral) em função da distribuição populacional e do valor esperado para as probabilidades de seleção da amostra. Foram considerados os casos em que a distribuição das probabilidades de seleção da amostra é conhecida e também os casos em que essa informação não está disponível para quem está fazendo a análise dos dados.

Para esses casos de falta de informação, sob algumas condições de regularidade, o valor esperado das probabilidades de seleção da amostra pode ser modelado por um polinômio aproximador de baixo grau dependendo da variável resposta e das variáveis concomitantes via séries de Taylor. Com isso, é possível definir a distribuição amostral da variável resposta com os coeficientes desse polinômio aproximador incluídos entre os parâmetros a serem estimados no modelo.

Assim, considerando que o modelo válido para a população estudada (distribuição populacional) é especificado corretamente, se ao ajustar o modelo amostral todos os valores estimados para os coeficientes do polinômio aproximador forem iguais a zero, excluindo o termo independente do polinômio, então o modelo que melhor ajusta os dados é o modelo 
populacional e, portanto, a amostragem não é informativa. Caso contrário, se algum dos coeficientes for diferente de zero então existe um modelo que ajusta melhor os dados que o modelo populacional considerado. Nesse caso, a amostragem é informativa e o modelo amostral deve ser considerado na inferência dos dados. Observe que avaliar a informatividade do processo de amostragem utilizando os coeficientes do polinômio aproximador conforme descrito acima só é possível quando o modelo populacional é especificado corretamente.

Entretanto, avaliar se cada uma das estimativas para os parâmetros do polinômio aproximador podem ser consideradas iguais a zero, e consequentemente decidir se a amostragem é informativa ou ignorável, não é uma tarefa tão simples mesmo quando as estimativas dos parâmetros estão bem próximas de zero. Para decidir isso, é necessário avaliar quais são as consequências causadas por considerar cada uma dessas estimativas iguais a zero na distribuição amostral.

Para essa avaliação o presente trabalho propõe a utilização do teste de significância FBST para avaliar qual é o polinômio de menor grau que deve ser utilizado na distribuição amostral de forma que seja possível garantir estimativas satisfatórias para os parâmetros do modelo populacional. Essa avaliação consiste em comparar os polinômios de um determinado grau $d$ com o polinômio de grau $d+1$ para avaliar se o coeficiente do termo de grau $d+1$ pode ser considerado igual a zero. Em termos de teste de significância, isso é equivalente a considerar a distribuição amostral com o polinômio aproximador de grau $d+1$ e avaliar a hipótese precisa definida pelo coeficiente de termo de grau $d+1$ igual a zero.

Além disso, no presente trabalho foram realizadas simulações computacionais com o objetivo de avaliar: os efeitos práticos de ignorar o processo de seleção da amostra; a sensibilidade de modelar o valor esperado das probabilidades de inclusão da amostra aproximando por polinômios; o viés entre as estimativas aceitas e as estimativas rejeitadas pelo FBST. Essas simulações computacionais foram realizadas considerando 500 populações geradas aleatoriamente com distribuição normal e 6 métodos diferentes para selecionar amostras dessas populações sendo 5 deles informativos e o outro não informativo. Assim, de cada população gerada computacionalmente foram sorteadas 6 amostras e cada uma dessas amostras foi analisada considerando três abordagem diferente, são elas: 
i) O processo de amostragem é ignorado, ou seja, o modelo populacional é utilizado para estimar os parâmetros do modelo;

ii) A distribuição das probabilidades de seleção da amostra não está disponível para quem faz a análise dos dados. Nesse caso, o modelo amostral da variável resposta é ajustado modelando o valor esperado dessas probabilidades de seleção por polinômios aproximadores e o FBST é utilizado para decidir o polinômio de menor grau apropriado para a inferência dos dados;

iii) A distribuição das probabilidades de seleção da amostra é conhecida durante o processo de inferência e a distribuição amostral da variável resposta é definida utilizando essa informação.

Nestas simulações computacionais, os modelos de inferência utilizando a distribuição amostral, considerando desconhecida a distribuição do valor esperado das probabilidades de seleção da amostra, mostraram-se eficientes quando o grau do polinômio aproximador é suficientemente grande de forma a garantir que as estimativas fossem aceitas pelo FBST, pois foram obtidas estimativas com precisão semelhante ao modelo utilizando a distribuição amostral com a distribuição do valor esperado das probabilidades de seleção da amostra conhecida.

Nos casos em que as amostras foram selecionadas sob amostragem não informativa, todos os modelos utilizando a distribuição amostral conseguiram estimar os parâmetros com a mesma precisão que os modelos utilizando a distribuição populacional, pois nesses casos os paramêtros da função que modela o valor esperado das probabilidades de seleção da amostra foram estimados iguais a zero. Além disso nos casos em que foram utilizados polinômios aproximadores, o FBST aceitou todas as hipóteses que avaliavam se os coeficiente do polinômio aproximador eram iguais a zero.

Nos demais casos, as amostras foram selecionadas utilizando métodos de amostragem informativos e por isso as estimativas utilizando o modelo populacional apresentaram um viés elevado quando comparadas com as estimativas obtidas utilizando os modelos amostrais. Além disso, as estimativas encontradas utilizando uma aproximação polinomial com o grau do polinômio suficientemente grande para modelar o valor esperado das probabilidades de seleção da amostra ficaram bem próximas das estimativas obtidas considerando o valor esperado dessas probabilidades de seleção conhecido. 
Feito isso, foram comparadas também as estimativas aceitas com as estimativas rejeitadas utilizando o FBST para certificar que esse teste de significância está identificando corretamente as estimativas próximas do valor utilizado para gerar os dados populacionais. Essa comparação mostrou que o viés das estimativas aceitas foi sempre menor que o viés dos estimativas rejeitadas utilizando esse teste.

Por conseguinte, os objetivos iniciais do presente trabalho foram alcançados tendo como contribuições principais o desenvolvimento de uma metodologia intuitiva para avaliar se o processo de amostragem probabilística de uma pesquisa é informativo e, quando isso acontece, foram propostas maneiras de escolher um modelo que melhor ajusta os dados coletados considerando uma metodologia que não viola o Príncipio da Verossimilhança, permite a incorporação de experiência prévia e a opinião de especialistas, pode ser facilmente implementada utilizando modernas técnicas de otimização e integração numérica e permite o uso das ferramentas padrão de inferência bayesiana.

Além disso, é importante ressaltar que as simulações computacionais foram realizadas utilizando o software estatístico $R$ e o pacote de otimização ALGENCAN (implementado na linguagem de programação FORTRAN), ambos disponíveis tanto para Linux quanto para MAC OS e Windows.

Como consequência a este trabalho, pretendemos comparar os resultados obtidos para avaliar a informatividade do processo de seleção da amostra e também para decidir qual o polinômio de menor grau que ajusta os dados com precisão satisfatória utilizando o FBST com os testes de informatividade propostos por Pfeffermann [27] e por Pfeffermann e Sverchkov [29]. Além disso, a metodologia utilizada no presente trabalho para avaliar a informatividade do processo de amostragem pode ser estendida para os casos de nãoresposta ao item utilizando os modelos de inferência propostos por Pfeffermann e Sikov [33] para modelar os dados e o FBST para avaliar se a relação entre as probabilidades de não-resposta ao item ocorrida durante o processo de coleta dos dados e a variável resposta é significante.

O presente trabalho está dividido da seguinte maneira: no Capítulo 2 são apresentados formalmente os conceitos de amostragem informativa e não-resposta informativa, incluindo as condições necessárias para considerar que um desenho amostral e os casos de não-resposta sejam considerados informativos. Além disso, a metodologia proposta por Pfeffermann, Krieger e Rinott [28] para realizar inferência considerando esse tipo 
de amostragem e a extensão dessa metodologia para os casos de não-resposta informativa proposta por Pfeffermann e Sikov [33] também são discutidas. No Capítulo 3, são apresentados conceitos, propriedades e técnicas computacionais úteis na implementação do teste de significância para hipóteses precisas FBST. No Capítulo 4, são descritas as simulações computacionais realizadas no presente trabalho com o objetivo de avaliar o comportamento dos modelos de inferência sob amostragem informativa utilizando o valor de evidência do FBST. E por fim, no Apêndice, está uma cópia de um artigo ainda em desenvolvimento com os resultados desta tese. 


\section{Amostragem informativa}

Existe uma grande variedade de situações nas quais a análise de dados deve considerar o mecanismo de coleta dos dados e das respostas ao invés de simplesmente modelar diretamente os dados observados. Isso ocorre quando as probabilidades de seleção da amostra ou de não-resposta estão associadas com os valores da variável resposta do modelo, mesmo quando condicionadas às variáveis auxiliares.

$\mathrm{Na}$ amostragem estratificada e na amostragem por conglomerados, por exemplo, as unidades da amostra são selecionadas com probabilidades diferentes. Se essas probabilidades estão relacionadas com a variável resposta, o projeto amostral é informativo e o modelo apropriado para ajustar os dados da amostra coletada é diferente do modelo para ajustar os dados populacionais. Nesse caso, a informação que descreve como os dados foram coletados deve ser considerada no processo de inferência dos dados.

Pesquisas em que as probabilidades de não-resposta estão relacionadas com a variável resposta costumam ocorrer, por exemplo, em problemas de econometria em que o objetivo é estimar salários ou rendimentos. Nesse tipo de pesquisa é comum as probabilidades de não-resposta estarem diretamente relacionadas com salários e rendimentos, pois os indivíduos com maior renda tendem a evitar responder questões sobre isso.

O objetivo do presente capítulo é discutir condições suficientes para garantir que o modelo de seleção da amostra e o mecanismo de não-resposta possam ser ignorados no processo de inferência dos dados, além de maneiras apropriadas de realizar essa inferência quando pelo menos um desses dois processos é informativo. Para isso, apresentamos um método de inferência, proposto por Pfeffermann, Krieger e Rinott [28] para o caso de 
amostragem informativa e posteriormente discutido para os casos em que o mecanismo de não-resposta é informativo por Pfeffermann e Sikov [33], para estimar os parâmetros da distribuição populacional sob amostragem ou mecanismo de não-resposta informativos no qual é possível expressá-lo por um modelo de distribuição correspondente, explorando a relação entre os dois.

\subsection{Projetos amostrais informativos e ignoráveis}

Na presente Seção, apresentamos as definições e notações referentes ao processo de amostragem e não-resposta dos dados de uma pesquisa juntamente com as condições sob as quais esses processos podem ser ignorados no processo de inferência.

Considere uma população finita representada por $U=\{1,2, \ldots, N\}$ onde $N$, o tamanho da população, é conhecido. Associado com cada unidade $k \in U$, existe uma quantidade desconhecida $y_{k}$ e o vetor populacional contendo todas essas quantidades desconhecidas é representado por $\boldsymbol{y}^{T}=\left(y_{1}, \ldots, y_{N}\right)$, onde $\boldsymbol{y}$ é um vetor coluna e $\boldsymbol{y}^{T}$ é o seu transposto. Além disso, para cada unidade $k \in U$ são supostas $p$ quantidades $x_{k 1}, \ldots, x_{k p}$ representadas pelo vetor $\boldsymbol{x}_{k}^{T}=\left(x_{k 1}, \ldots, x_{k p}\right)$ contendo informações que podem ajudar a explicar a variável $y_{k}$. Denotamos por $X$ a matriz de ordem $N \times p$ cujas linhas são formadas pelos vetores $\boldsymbol{x}_{k}^{T}$.

Com o objetivo de obter informações sobre uma função linear $A^{T} y$, onde $A^{T}=$ $\left(a_{1}, \ldots, a_{N}\right)$ é conhecida, uma amostra $S \subset U$ com $n$ unidades de $U$ é selecionada utilizando em cada unidade $k \in U$ as quantidades $z_{1}, \ldots, z_{m}$ representadas pelo vetor $\boldsymbol{z}_{k}^{T}=\left(z_{1}, \ldots, z_{m}\right)$ que contem informações sobre o processo de seleção da amostra. Assim, denotamos por $Z_{U}$ a matriz de ordem $N \times m$ cujas linhas são formadas pelos vetores $\boldsymbol{z}_{k}^{T}$ para todo os valores de $k \in U$. Esta matriz $Z_{U}$ pode conter variáveis determinando estratos, conglomerados, variáveis quantitativas, algumas ou todas as covariáveis $X$ e, em casos especiais, o vetor $\boldsymbol{y}$ também. Em geral, os valores populacionais $Z_{U}$ são conhecidos pelo profissional que planeja e realiza o processo de amostragem, não sendo conhecidos necessariamente por quem faz a análise dos dados.

Para representar os elementos da amostra $S$ utiliza-se o vetor de inclusão $\boldsymbol{i}=\left(i_{1}, i_{2}, \ldots, i_{N}\right)$ das variáveis indicadoras do processo de amostragem, isto é, $i_{k}=1$ se 
a unidade $k \in U$ é selecionada na amostragem e $i_{k}=0$ caso contrário.

Quando os casos de não-respostas no processo de amostragem são considerados, definese para cada $k \in U$ o vetor $\boldsymbol{l}_{k}$ contendo as variáveis explicativas do processo de nãoresposta. Dessa maneira, denotamos por $L$ a matrix cujas linhas são formadas pelos vetores $\boldsymbol{l}_{k}$, com $k \in U$, contendo as variáveis referentes à não-resposta. Essa matriz $L$ referente ao processo de não-resposta é raramente conhecida, apesar de poder conter elementos de $\boldsymbol{y}, X$ e $Z$.

Assim, qualquer subconjunto $S=\left\{k \in U \mid i_{k}=1\right\}$ da população $U$ caracteriza uma amostra dessa população e para cada amostra $S$ define-se $\left[\boldsymbol{y}_{s}, X_{s}, Z_{s}, L_{s}\right]=$ $\left\{\left(y_{k}, \boldsymbol{x}_{k}, \boldsymbol{z}_{k}, \boldsymbol{l}_{k}\right) \mid k \in S\right\}$ e $\left[\boldsymbol{y}_{\bar{s}}, X_{\bar{s}}, Z_{\bar{s}}, L_{\bar{s}}\right]=\left\{\left(y_{k}, \boldsymbol{x}_{k}, \boldsymbol{z}_{k}, \boldsymbol{l}_{k}\right) \mid k \notin S\right\}$. Observe que $S=S(\boldsymbol{i})$.

Vamos considerar $\boldsymbol{y}^{T}$ como realização do vetor aleatório $\boldsymbol{Y}^{T}=\left(Y_{1}, \ldots, Y_{N}\right)$ cuja distribuição depende de um parâmetro $\boldsymbol{\theta}$ desconhecido. Assim, quando $\boldsymbol{\theta}$ é conhecido, $Y_{1}, \ldots, Y_{N}$ são mutuamente independentes. Depois de considerar $\boldsymbol{y}$ como realização de um vetor aleatório, o objetivo do estudo é predizer o valor de $\sum_{k \in \bar{S}} A_{k} y_{k}$, a parte não observada de $A^{T} \boldsymbol{y}$, utilizando toda a informação das variáveis auxiliares contida na matriz $X$. Por esse motivo, é desejável considerar modelos que relacionam $\boldsymbol{y} \operatorname{com} X$.

Para analisar os métodos de seleção da amostra considere por conveniência o caso de resposta completa, a extensão dos resultados para o caso de não-resposta segue de maneira análoga. Assim, considere o modelo de probabilidade conjunta dividido em duas partes: o modelo para os dados completos, $\boldsymbol{y}$, (incluindo componentes observadas e não observadas) condicionado às variáveis auxiliares representadas pela matriz $X$ e ao conjunto de parâmetros $\boldsymbol{\theta}$; e o modelo para o vetor de inclusão, $\boldsymbol{i}$, condicionado a $X, \boldsymbol{y}$ e ao conjunto de parâmetros $\boldsymbol{\psi}$. Com isso, a verossimilhança dos dados completos é definida como o produto das verossimilhanças desses dois fatores, isto é,

$$
f(\boldsymbol{y}, \boldsymbol{i} \mid X, \boldsymbol{\theta}, \boldsymbol{\psi})=f(\boldsymbol{y} \mid X, \boldsymbol{\theta}) f(\boldsymbol{i} \mid X, \boldsymbol{y}, \boldsymbol{\psi}) .
$$

A expressão (2.1) é útil na construção do modelo de probabilidade, mas não é a verossimilhança dos dados a menos que $\boldsymbol{y}$ seja completamente observado. Assim, os dados que são realmente conhecidos consistem do conjunto $\left(\boldsymbol{y}_{s}, \boldsymbol{i}\right)$ e a verossimilhança apropriada para inferência Bayesiana, denominada verossimilhança dos dados observados, pode ser obtida integrando (2.1) sobre os itens não amostrados $\boldsymbol{y}_{\bar{s}}$ considerando as unidades 
amostradas fixadas, isto é,

$$
f\left(\boldsymbol{y}_{s}, \boldsymbol{i} \mid X, \boldsymbol{\theta}, \boldsymbol{\psi}\right)=\int f\left(\boldsymbol{y}_{s}, \boldsymbol{y}_{\bar{s}} \mid X, \boldsymbol{\theta}\right) f\left(\boldsymbol{i} \mid X, \boldsymbol{y}_{s}, \boldsymbol{y}_{\bar{s}}, \boldsymbol{\psi}\right) d \boldsymbol{y}_{\bar{s}}
$$

Observe que a expressão (2.2) é muito geral e não impõe nenhuma restrição no mecanismo de seleção da amostra. Se o mecanismo de seleção da amostra é ignorado, então a verosssimilhança dos dados observados pode ser escrita como

$$
f\left(\boldsymbol{y}_{s} \mid X, \boldsymbol{\theta}\right)=\int f\left(\boldsymbol{y}_{s}, \boldsymbol{y}_{\bar{s}} \mid X, \boldsymbol{\theta}\right) d \boldsymbol{y}_{\bar{s}}
$$

Assim, ignorar o mecanismo de seleção da amostra nesse caso significa omitir $f\left(\boldsymbol{i} \mid X, \boldsymbol{y}_{s}, \boldsymbol{y}_{\bar{s}}, \boldsymbol{\psi}\right)$ no lado direito de (2.2), considerando a inferência baseada somente em (2.3). Claramente a inferência considerando (2.2) geralmente não é a mesma que a inferência baseada em (2.3) onde os possíveis efeitos do mecanismo de seleção da amostra são considerados.

Seguindo Rubin [34], [35] e [36], dizemos que um projeto amostral (ou mecanismo de seleção da amostra) é ignorável se a inferência baseada na função de densidade de probabilidade de $Y \mid X$ descrita em (2.3) é equivalente a inferência baseada em (2.2). Neste caso, as probabilidades de seleção da amostra dependem somente de $X$ de maneira que

$$
f\left(\boldsymbol{i} \mid X, \boldsymbol{y}_{s}, \boldsymbol{y}_{\bar{s}}, \boldsymbol{\psi}\right)=f(\boldsymbol{i} \mid X, \boldsymbol{\psi})
$$

Na prática, dizer que um projeto amostral é ignorável não significa que ele não fornece informações úteis, mas que as probabilidades de seleção da amostra não fornecem informações adicionais daquelas já fornecidas pelo processo de amostragem.

A condição (2.4) é uma condição muito forte para garantir ignorabilidade do mecanismo de seleção da amostra. Em um artigo sobre dados faltantes, Rubin [34] estabelece duas condições necessárias e suficientes sob as quais o processo de dados faltantes pode ser ignorado. Uma delas é a condição de dados faltantes ao acaso (missing at random),

$$
f(\boldsymbol{i} \mid X, \boldsymbol{y}, \boldsymbol{\psi})=f\left(\boldsymbol{i} \mid X, \boldsymbol{y}_{s}, \boldsymbol{\psi}\right)
$$

isto é, $f(\boldsymbol{i} \mid X, \boldsymbol{y}, \psi)$ avaliada nos dados observados $\left(X, \boldsymbol{i}, \boldsymbol{y}_{s}\right)$ independe de $\boldsymbol{y}_{\bar{s}}$.

A segunda condição é a de parâmetros distintos, que é satisfeita quando os parâmetros $\boldsymbol{\psi}$ do processo de dados faltantes são independentes dos parâmetros $\boldsymbol{\theta}$ do processo de 
geração dos dados, condicionados aos valores das covariáveis $X$, isto é,

$$
f(\boldsymbol{\psi} \mid X, \boldsymbol{\theta})=f(\boldsymbol{\psi} \mid X)
$$

É importante compreender que apesar de toda inferência ser realizada condicionada aos dados observados, não é correto afirmar que a maneira como esses dados foram coletados não faz diferença na inferência. A questão central é que a definição de dados observados deve incluir informações de como esses dados foram coletados, pois em muitas situações essas informações têm importância fundamental em como esses valores devem ser interpretados na modelagem da função de verossimilhança.

\subsection{Mecanismos de não-resposta informativos e ig- noráveis}

Na presente Seção, apresentamos as condições sob as quais o mecanismo de nãoresposta de uma pesquisa pode ser considerado ignorável. Essas condições foram propostas por Little [17] como uma extensão dos resultados de Rubin [34] distinguindo entre a seleção da amostra e o processo de não-resposta. Posteriormente, Sugden e Smith [40] estabeleceram condições sob as quais um processo de amostragem que depende das variáveis de desenho $Z$ pode ser considerado ignorável, conhecendo somente informações parciais do projeto amostral.

Para analisar os mecanismos de não-resposta do modelo, é conveniente dividir a variável resposta $\boldsymbol{y}$ em dois grupos $\boldsymbol{u}$ e $\boldsymbol{v}$, onde $\boldsymbol{u}$ são as variáveis completamente observadas na amostragem e $\boldsymbol{v}$ são as variáveis sujeitas à não-resposta.

O padrão de não-respostas de $\boldsymbol{y}$ é descrito pelo vetor indicador de respostas $\boldsymbol{r}$, onde $r_{k}=1$ se a unidade $k \in S$ responde e $r_{k}=0$ caso contrário. Os valores amostrados de $\boldsymbol{u}$, $\boldsymbol{v}$ e $\boldsymbol{r}$ são denotados por $\boldsymbol{u}_{s}, \boldsymbol{v}_{s}$ e $\boldsymbol{r}_{s}$, respectivamente, enquanto os valores não amostrados são denotados por $\boldsymbol{u}_{\bar{s}}, \boldsymbol{v}_{\bar{s}}$ e $\boldsymbol{r}_{\bar{s}}$, respectivamente. Além disso, os valores amostrados para $\boldsymbol{v}_{s}$ podem ser divididos em valores respondentes $\boldsymbol{v}_{s r}$ e dados faltantes $\boldsymbol{v}_{s \bar{r}}$.

Para avaliar o mecanismo de não-resposta, vamos considerar novamente a verossimilhança dos dados completos. Assim, 


$$
\begin{aligned}
f(\boldsymbol{y}, \boldsymbol{i}, \boldsymbol{r} \mid Z, \boldsymbol{\theta}, \boldsymbol{\psi}, \boldsymbol{\phi}) & =f(\boldsymbol{u}, \boldsymbol{v}, \boldsymbol{i}, \boldsymbol{r} \mid Z, \boldsymbol{\theta}, \boldsymbol{\psi}, \boldsymbol{\phi}) \\
& =f(\boldsymbol{u}, \boldsymbol{v} \mid Z, \boldsymbol{\theta}) f(\boldsymbol{i} \mid Z, \boldsymbol{u}, \boldsymbol{v}, \boldsymbol{\psi}) f(\boldsymbol{r} \mid Z, \boldsymbol{u}, \boldsymbol{v}, \boldsymbol{i}, \boldsymbol{\phi}) .
\end{aligned}
$$

Os dois primeiros fatores do lado direito de (2.7) são análogos a (2.1) e o último fator modela o padrão de não-respostas $\boldsymbol{r}$ através da distribuição condicional de $\boldsymbol{r}$ dado $(Z, \boldsymbol{u}, \boldsymbol{v}, \boldsymbol{i})$ e indexados pelo conjunto de parâmetros $\phi$.

Assim, a distribuição dos dados $\left(\boldsymbol{i}, \boldsymbol{u}_{s}, \boldsymbol{r}_{s}, \boldsymbol{v}_{s r}\right)$ pode ser obtida integrando (2.7) sobre as componentes faltantes de $\boldsymbol{u}, \boldsymbol{r}$ e $\boldsymbol{v}$, denotadas por $\boldsymbol{u}_{\bar{s}}, \boldsymbol{r}_{\bar{s}}, \boldsymbol{v}_{\bar{s}}$ e $\boldsymbol{v}_{s \bar{r}}$. Logo,

$$
\begin{aligned}
f\left(\boldsymbol{i}, \boldsymbol{u}_{s}, \boldsymbol{r}_{s}, \boldsymbol{v}_{s r} \mid Z, \boldsymbol{\theta}, \boldsymbol{\psi}, \boldsymbol{\phi}\right)= & \int f\left(\boldsymbol{u}_{s}, \boldsymbol{u}_{\bar{s}}, \boldsymbol{v}_{s r}, \boldsymbol{v}_{s \bar{r}}, \boldsymbol{v}_{\bar{s}} \mid Z, \boldsymbol{\theta}\right) \\
& \times f\left(\boldsymbol{i} \mid Z, \boldsymbol{u}_{s}, \boldsymbol{u}_{\bar{s}}, \boldsymbol{v}_{s r}, \boldsymbol{v}_{s \bar{r}}, \boldsymbol{v}_{\bar{s}}, \boldsymbol{\psi}\right) \\
& \times f\left(\boldsymbol{r}_{s} \mid Z, \boldsymbol{u}_{s}, \boldsymbol{u}_{\bar{s}}, \boldsymbol{v}_{s r}, \boldsymbol{v}_{\bar{s}}, \boldsymbol{v}_{\bar{s}}, \boldsymbol{i}, \boldsymbol{\phi}\right) \\
& \times d \boldsymbol{u}_{\bar{s}} d \boldsymbol{v}_{\bar{s}} d \boldsymbol{v}_{s \bar{r}},
\end{aligned}
$$

onde a distribuição das não-respostas está restrita as unidades amostradas $\boldsymbol{r}_{s}$.

A maioria dos métodos para tratar casos de não-resposta são baseados em modelos que não consideram conjuntamente distribuições para a amostra e para as não-respostas e são restritos a distribuição marginal dos valores observados $\boldsymbol{u}_{s}$ e $\boldsymbol{v}_{s r}$. Nesse caso, tem-se

$$
f\left(\boldsymbol{u}_{s}, \boldsymbol{v}_{s r} \mid Z, \boldsymbol{\theta}\right)=\int f\left(\boldsymbol{u}_{s}, \boldsymbol{u}_{\bar{s}}, \boldsymbol{v}_{s r}, \boldsymbol{v}_{s \bar{r}}, \boldsymbol{v}_{\bar{s}} \mid Z, \boldsymbol{\theta}\right) d \boldsymbol{u}_{\bar{s}} d \boldsymbol{v}_{\bar{s}} d \boldsymbol{v}_{s \bar{r}}
$$

Estendendo a terminologia utilizada na Seção anterior, diz-se que um projeto amostral e o mecanismo de não-resposta são ignoráveis se a inferência sobre $\boldsymbol{\theta}$ baseada em (2.9) é equivalente a inferência baseada na verossimilhança dos dados completos (2.8). Consequentemente, as inferências sobre $\boldsymbol{\theta}$ baseadas em (2.8) e (2.9) são equivalentes se estas expressões diferem somente por fatores independentes de $\boldsymbol{\theta}$.

Em virtude disso, Little [17] utilizou a teoria de Rubin [34] para estabelecer as seguintes condições suficientes para garantir ignorabilidade do mecanismo de amostragem e de nãoresposta:

1. $\boldsymbol{\theta}, \boldsymbol{\psi}$ e $\boldsymbol{\phi}$ são conjuntos de parâmetros distintos. Para teoria bayesiana, eles são distribuidos independentemente a priori. 
2. A distribuição amostral $f(\boldsymbol{i} \mid Z, \boldsymbol{u}, \boldsymbol{v}, \boldsymbol{\psi})$ não depende dos itens não observados $\boldsymbol{u}_{\bar{s}}$, $\boldsymbol{v}_{\bar{s}}$ e $\boldsymbol{v}_{s \bar{r}}$.

3. A distribuição das não-respostas das unidades amostradas $f\left(\boldsymbol{r}_{s} \mid Z, \boldsymbol{u}, \boldsymbol{v}, \boldsymbol{i}, \boldsymbol{\phi}\right)$ não depende dos itens não observados $\boldsymbol{u}_{\bar{s}}, \boldsymbol{v}_{\bar{s}}$ e $\boldsymbol{v}_{\bar{s} \bar{r}}$.

Essas condições podem ser enfraquecidas se $\boldsymbol{\theta}$ tiver algumas propriedades especiais. Por exemplo, suponha que a distribuição conjunta de $\boldsymbol{u}_{s}$ e $\boldsymbol{v}_{s}$ pode ser fatorada como

$$
f\left(\boldsymbol{u}_{s}, \boldsymbol{v}_{s} \mid Z, \boldsymbol{\theta}\right)=f\left(\boldsymbol{u}_{s} \mid Z, \boldsymbol{\theta}_{1}\right) f\left(\boldsymbol{v}_{s} \mid \boldsymbol{u}_{s}, Z, \boldsymbol{\theta}_{2}\right)
$$

Se $\boldsymbol{\theta}_{1}$ e $\boldsymbol{\theta}_{2}$ são parâmetros distintos (no caso de inferência bayesiana, $\boldsymbol{\theta}_{1}$ e $\boldsymbol{\theta}_{2}$ são independentes a priori), então o mecanismo de amostragem e de não-respostas pode ser ignorado para inferência sobre $\boldsymbol{\theta}_{1}$ quando a condição 1 é válida juntamente com as duas seguintes condições (que são mais fracas que as condições 2 e 3)

4. A distribuição amostral $f(\boldsymbol{i} \mid Z, \boldsymbol{u}, \boldsymbol{v}, \boldsymbol{\psi})$ não depende dos itens não amostrados $\boldsymbol{u}_{\bar{s}}$ e $\boldsymbol{v}_{\bar{s}}$.

5. A distribuição respondente $f\left(\boldsymbol{r}_{s} \mid Z, \boldsymbol{u}, \boldsymbol{v}, \boldsymbol{i}, \boldsymbol{\phi}\right)$ não depende dos itens não amostra$\operatorname{dos} \boldsymbol{u}_{\bar{s}}$ e $\boldsymbol{v}_{\bar{s}}$

Consequentemente, os mecanismos de não-resposta para $\boldsymbol{v}_{s}$ que dependem dos itens amostrados e não dependem dos valores faltantes $\boldsymbol{v}_{s \bar{r}}$, apesar de não poderem ser ignorados para inferência sobre $\boldsymbol{\theta}_{2}$, podem ser ignorados em inferências sobre $\boldsymbol{\theta}_{1}$, o parâmetro da distribuição de $\boldsymbol{u}_{s}$.

Exemplo 2.2.1. Considere uma pesquisa com amostragem estratificada tal que $Z$ é uma variável indicando o estrato, que pode ser por exemplo uma região geográfica, e cada estrato $J$ contém $N_{J}$ indivíduos com $n_{J}$ deles sendo sorteados por amostragem aleatória simples. Além disso, considere que existem duas variáveis: $\boldsymbol{u}$, indicando escolaridade e completamente observada, e $\boldsymbol{v}$, indicando renda familiar e parcialmente observada. Então visto que a distribuição $f(\boldsymbol{i} \mid Z, \boldsymbol{u}, \boldsymbol{v}, \boldsymbol{\psi})$ é conhecida e depende somente de $Z$, as condições 2 e 4 são satisfeitas. Por outro lado, se a distribuição $f\left(\boldsymbol{r}_{s} \mid Z, \boldsymbol{u}, \boldsymbol{v}, \boldsymbol{i}, \boldsymbol{\phi}\right)$ depende da região e da escolaridade e não depende da variável resposta (renda domiciliar), então a condição 3 é satisfeita e o mecanismo de não-resposta é ignorável apesar dos valores da variável 
resposta não serem necessariamente uma amostra aleatória das unidades selecionadas em cada região.

Para resumir as condições discutidas durante a presente Seção, pode-se dizer que dada a probabilidade de seleção da amostra, para a maioria dos problemas a distribuição respondente pode ser ignorada se ela não depende dos valores dos itens que são faltantes para algumas unidades. Quase todos os procedimentos para tratar não-resposta faz essa consideração, mesmo que implicitamente. Em particular, a probabilidade de não-resposta é considerada constante dentro de subclasses definidas pelos valores das variáveis da amostragem $Z$ ou em variáveis completamente observadas $\boldsymbol{u}_{s}$.

\subsection{Distribuição marginal amostral}

Na presente Seção, a função de densidade de probabilidade populacional poderá depender de valores conhecidos de variáveis auxiliares $\boldsymbol{x}_{i}$ tais que $Y_{i} \mid \boldsymbol{X}_{i}=\boldsymbol{x}_{i} \sim f_{p}\left(y_{i} \mid \boldsymbol{x}_{i}, \boldsymbol{\theta}\right)$. Os vetores $\boldsymbol{x}_{i}$ podem incluir algumas variáveis do projeto amostral bem como outras variáveis auxiliares.

Segundo Pfeffermann, Krieger e Rinott [28], quando a função de densidade de probabilidade populacional depende de variáveis concominantes, a função de densidade de probabilidade amostral de $Y_{i}$ considerando o caso de resposta completa pode ser escrita, utilizando-se o Teorema de Bayes, como

$$
\begin{aligned}
f_{s}\left(y_{i} \mid \boldsymbol{x}_{i}, \boldsymbol{\theta}, \boldsymbol{\psi}\right) & =f\left(y_{i} \mid \boldsymbol{x}_{i}, \boldsymbol{\theta}, \boldsymbol{\psi}, I_{i}=1\right) \\
& =\operatorname{Pr}\left(I_{i}=1 \mid y_{i}, \boldsymbol{x}_{i}, \boldsymbol{\psi}\right) f_{p}\left(y_{i} \mid \boldsymbol{x}_{i}, \boldsymbol{\theta}\right) / \operatorname{Pr}\left(I_{i}=1 \mid \boldsymbol{x}_{i}, \boldsymbol{\psi}\right) .
\end{aligned}
$$

Segue da equação (2.11) que a função de densidade de probabilidade populacional é diferente da função de densidade de probabilidade amostral a menos que $\operatorname{Pr}\left(I_{i}=1 \mid y_{i}, \boldsymbol{x}_{i}, \boldsymbol{\psi}\right)=\operatorname{Pr}\left(I_{i}=1 \mid \boldsymbol{x}_{i}, \boldsymbol{\psi}\right)$ para todo $y_{i}$ e nesse caso a amostragem condicionada a $\boldsymbol{x}_{i}$ não é informativa.

Como os parâmetros da função de densidade de probabilidade amostral incluem os parâmetros da função de densidade de probabilidade populacional, esses parâmetros podem ser estimados calculando-se a máxima verossimilhança ou utilizando qualquer outro 
método de estimação de medidas amostrais utilizando a distribuição amostral. A principal vantagem de fazer a inferência utilizando a distribuição amostral é que ela permite a utilização de procedimentos eficientes de inferência.

Observe que $\operatorname{Pr}\left(I_{i}=1 \mid y_{i}, \boldsymbol{x}_{i}, \boldsymbol{\psi}\right)$ geralmente não é a mesma que a probabilidade de seleção $\pi_{i}=\operatorname{Pr}\left(I_{i}=1 \mid Z_{U}\right)$ que depende de todos valores populacionais de $Z$. Considerando essas probabilidades $\pi_{i}$ como realizações de variáveis aleatórias, a seguinte relação é válida

$\operatorname{Pr}\left(I_{i}=1 \mid y_{i}, \boldsymbol{x}_{i}, \boldsymbol{\psi}\right)=\int \operatorname{Pr}\left(I_{i}=1 \mid y_{i}, \boldsymbol{x}_{i}, \boldsymbol{\psi}, \pi_{i}\right) f_{p}\left(\pi_{i} \mid y_{i}, \boldsymbol{x}_{i}, \boldsymbol{\psi}\right) d \pi_{i}=E_{p}\left(\pi_{i} \mid y_{i}, \boldsymbol{x}_{i}, \boldsymbol{\psi}\right)$,

pois $\operatorname{Pr}\left(I_{i}=1 \mid y_{i}, \boldsymbol{x}_{i}, \boldsymbol{\psi}, \pi_{i}\right)=\pi_{i}$. Substituindo (2.12) em (2.11), tem-se uma expressão alternativa para a função de densidade de probabilidade amostral

$$
f_{s}\left(y_{i} \mid \boldsymbol{x}_{i}, \boldsymbol{\theta}, \boldsymbol{\psi}\right)=E_{p}\left(\pi_{i} \mid y_{i}, \boldsymbol{x}_{i}, \boldsymbol{\psi}\right) f_{p}\left(y_{i} \mid \boldsymbol{x}_{i}, \boldsymbol{\theta}\right) / E_{p}\left(\pi_{i} \mid \boldsymbol{x}_{i}, \boldsymbol{\psi}\right)
$$

onde o valor esperado no denominador segue de maneira análoga a (2.12).

Uma característica importante de (2.13) é que para uma dada função de densidade de probabilidade populacional, a função de densidade de probabilidade amostral correspondente é complemente determinada pela esperança condicional $E_{p}\left(\pi_{i} \mid y_{i}, \boldsymbol{x}_{i}, \boldsymbol{\psi}\right)$, já que $E_{p}\left(\pi_{i} \mid \boldsymbol{x}_{i}, \boldsymbol{\psi}\right)=\int E_{p}\left(\pi_{i} \mid y_{i}, \boldsymbol{x}_{i}, \boldsymbol{\psi}\right) f_{p}\left(y_{i} \mid \boldsymbol{x}_{i}, \boldsymbol{\theta}\right) d y_{i}$.

Quando a função de densidade de probabilidade populacional não depende de variáveis concomitantes, tem-se que $Y_{i} \sim f_{p}\left(y_{i} \mid \boldsymbol{\theta}\right)$ e a relação (2.13) é reduzida para

$$
f_{s}\left(y_{i} \mid \boldsymbol{\theta}, \boldsymbol{\psi}\right)=E_{p}\left(\pi_{i} \mid y_{i}, \boldsymbol{\psi}\right) f_{p}\left(y_{i} \mid \boldsymbol{\theta}\right) / E_{p}\left(\pi_{i} \mid \boldsymbol{\psi}\right) .
$$

Nesse caso, o denominador é um número fixo, embora possa depender de parâmetros desconhecidos. 


\section{Exemplo 2.3.1. Modelos com regressão linear}

Considere a distribuição populacional dada por

$$
f_{p}\left(y_{i} \mid \boldsymbol{x}_{i}, \beta_{0}, \boldsymbol{\beta}, \sigma^{2}\right)=N\left(\beta_{0}+\boldsymbol{x}_{i}^{T} \boldsymbol{\beta}, \sigma^{2}\right),
$$

e que as probabilidades de inclusão na amostra tem valor esperado

$$
E_{p}\left(\pi_{i} \mid y_{i}, \boldsymbol{x}_{i}, \alpha_{1}, \alpha_{2}\right)=\exp \left[\alpha_{1} y_{i}+\alpha_{2} y_{i}^{2}+h\left(\boldsymbol{x}_{i}\right)\right],
$$

para alguma função $h\left(\boldsymbol{x}_{i}\right)$.

Utilizando a relação (2.13), tem-se que a distribuição amostral é dada por

$$
f_{s}\left(y_{i} \mid \boldsymbol{x}_{i}, \beta_{0}, \boldsymbol{\beta}, \sigma^{2}, \alpha_{1}, \alpha_{2}\right)=N\left(\left(\beta_{0}+\alpha_{1} \sigma^{2}+\boldsymbol{x}_{i}^{T} \boldsymbol{\beta}\right) / \tau, \sigma^{2} / \tau\right),
$$

onde $\tau=1-2 \alpha_{2} \sigma^{2}$.

Observe que apesar dos valores amostrais ainda terem distribuição normal, $E_{s}\left(y_{i} \mid \boldsymbol{x}_{i}, \beta_{0}, \boldsymbol{\beta}, \sigma^{2}, \alpha_{1}, \alpha_{2}\right)=\left(\beta_{0}+\alpha_{1} \sigma^{2}+\boldsymbol{x}_{i}^{T} \boldsymbol{\beta}\right) / \tau \neq \beta_{0}+\boldsymbol{x}_{i}^{T} \boldsymbol{\beta}=E_{p}\left(y_{i} \mid \boldsymbol{x}_{i}, \beta_{0}, \boldsymbol{\beta}, \sigma^{2}\right) e$ a variância também muda em função do fator fixo $\tau$.

No caso em que $\alpha_{2}=0$, a regressão considerando a distribuição populacional é a mesma, exceto pelo intercepto que passa a ser $\left(\beta_{0}+\alpha_{1} \sigma^{2}\right)$. Se $\alpha_{1}$ também é nulo, a amostragem não é informativa e os modelos populacional e amostral coincidem.

\section{Exemplo 2.3.2. Modelos Gama sob amostragem com probabilidade proporci- onal ao tamanho}

Considere que a distribuição populacional é Gama com parâmetro de forma a e média $\mu_{i}$, tal que

$$
f_{p}\left(y_{i} \mid \alpha, \mu_{i}\right) \propto y_{i}^{\alpha-1} \exp \left(-\alpha y_{i} / \mu_{i}\right)
$$

Se as probabilidades de seleção da amostra tem valores esperados $E_{p}\left(\pi_{i} \mid y_{i}\right) \propto y_{i}$ então, utilizando a relação (2.13), a distribuição amostral de $Y_{i}$ é também uma distribuição Gama com parâmetro de forma $(\alpha+1)$ e média $\mu_{i}(\alpha+1) \alpha$.

Considerando $\log \left(\mu_{i}\right)=\beta_{0}+\boldsymbol{x}_{i}^{T} \boldsymbol{\beta}$ com $\boldsymbol{x}_{i}$ um vetor de dados conhecidos, então

$$
E_{s}\left(y_{i} \mid \boldsymbol{x}_{i}, \alpha, \mu_{i}, \beta_{0}, \boldsymbol{\beta}\right)=\mu_{i} \frac{\alpha+1}{\alpha}=\exp \left[\beta_{0}+\log \left(\frac{\alpha+1}{\alpha}\right)+\boldsymbol{x}_{i}^{T} \boldsymbol{\beta}\right] .
$$

Observe que nesse caso somente o intercepto das distribuições populacional e amostral é diferente. 


\section{Exemplo 2.3.3. Modelos com regressão logística}

Considere $\boldsymbol{Y}$ uma variável de resposta categorizada assumindo valores $0,1, \ldots, L-1$, $X$ o conjunto de variável concomitantes e suponha que $\operatorname{Pr}(\boldsymbol{Y}=l \mid X, \boldsymbol{\alpha}, \boldsymbol{\beta})$ pode ser modelado usando regressão logística de forma que

$$
\operatorname{Pr}\left(y_{i}=l \mid \boldsymbol{x}_{i}, \boldsymbol{\alpha}, \boldsymbol{\beta}\right)=\exp \left(\alpha_{l}+\boldsymbol{x}_{i}^{T} \boldsymbol{\beta}_{l}\right) / \sum_{j=0}^{L-1} \exp \left(\alpha_{j}+\boldsymbol{x}_{i}^{T} \boldsymbol{\beta}_{j}\right),
$$

onde $\alpha_{0}=0$ e $\beta_{0}=0$ para garantir unicidade. Assim, o modelo (2.18) define a distribuição populacional antes do processo de amostragem.

Suponha que a seleção da amostra é realizada por amostragem de Poisson com probabilidades $\operatorname{Pr}\left(I_{i}=1 \mid y_{i}=l, \boldsymbol{x}_{i}\right)=P_{l}$, para cada $l=0, \ldots, L-1$. Assim,

$$
\begin{aligned}
\operatorname{Pr}\left(y_{i}=l \mid I_{i}=1, \boldsymbol{x}_{i}, \boldsymbol{\alpha}, \boldsymbol{\beta}\right) & =P_{l} \exp \left(\alpha_{l}+\boldsymbol{x}_{i}^{T} \boldsymbol{\beta}_{l}\right) / \sum_{j=0}^{L-1} P_{j} \exp \left(\alpha_{j}+\boldsymbol{x}_{i}^{T} \boldsymbol{\beta}_{j}\right) \\
& =\exp \left(\gamma_{l}+\boldsymbol{x}_{i}^{T} \boldsymbol{\beta}_{l}\right) / \sum_{j=0}^{L-1} \exp \left(\gamma_{j}+\boldsymbol{x}_{i}^{T} \boldsymbol{\beta}_{j}\right)
\end{aligned}
$$

onde $\gamma_{0}=0$ e $\gamma_{k}=\left[\log \left(P_{k} / P_{0}\right)+\alpha_{k}\right]$, para $k>0$.

Portanto, de acordo com (2.19), a distribuição amostral é novamente logística com o mesmo coeficiente de inclinação que a distribuição populacional e com diferentes interceptos. Obviamante, quando $P_{k}=P_{0}$ para todo $k$, as distribuições populacional e amostral são iguais.

\subsection{Distribuição marginal respondente}

A definição de função de densidade de probabilidade amostral dada por (2.13) pode ser estendida, considerando-se o caso de não-resposta, para distinguir entre a seleção da amostra e a probabilidade de não-resposta. Para isso, considere novamente $\boldsymbol{r}$ o vetor indicador de respostas, isto é, $r_{i}=1$ se a unidade $i \in S$ responder e $r_{i}=0$ caso contrário. Geralmente a probabilidade de resposta $\operatorname{Pr}\left(r_{i}=1 \mid I_{i}=1\right)$ é desconhecida e só pode ser estimada utilizando hipóteses estritas.

Segundo Pfeffermann e Sikov [33], considerando a variável indicadora de resposta $\boldsymbol{r}$, a 
função de densidade de probabilidade respondente é definida por

$$
\begin{aligned}
f_{r}\left(y_{i} \mid \boldsymbol{x}_{i}, \boldsymbol{\theta}, \boldsymbol{\psi}, \boldsymbol{\phi}\right) & =f\left(y_{i} \mid \boldsymbol{x}_{i}, \boldsymbol{\theta}, \boldsymbol{\psi}, \boldsymbol{\phi}, I_{i}=1, R_{i}=1\right) \\
& =\frac{\operatorname{Pr}\left(R_{i}=1 \mid y_{i}, \boldsymbol{x}_{i}, \boldsymbol{\phi}, I_{i}=1\right) f_{s}\left(y_{i} \mid \boldsymbol{x}_{i}, \boldsymbol{\theta}, \boldsymbol{\psi}\right)}{\operatorname{Pr}\left(R_{i}=1 \mid \boldsymbol{x}_{i}, \boldsymbol{\phi}, I_{i}=1\right)} \\
& =\frac{\operatorname{Pr}\left(R_{i}=1 \mid y_{i}, \boldsymbol{x}_{i}, \boldsymbol{\phi}, I_{i}=1\right) \operatorname{Pr}\left(I_{i}=1 \mid y_{i}, \boldsymbol{x}_{i}, \boldsymbol{\psi}\right) f_{p}\left(y_{i} \mid \boldsymbol{x}_{i}, \boldsymbol{\theta}\right)}{\operatorname{Pr}\left(R_{i}=1 \mid \boldsymbol{x}_{i}, \boldsymbol{\phi}, I_{i}=1\right) \operatorname{Pr}\left(I_{i}=1 \mid \boldsymbol{x}_{i}, \boldsymbol{\psi}\right)} .
\end{aligned}
$$

As funções de densidade de probabilidade (2.13), (2.20) e (2.21) referem-se à distribuição marginal da medida $y_{i}$. Essas definições generalizam a função de densidade de probabilidade conjunta de duas ou mais medidas associadas com diferentes unidades.

A modelagem das probabilidades de não-resposta foi feita por Greenlees, Reece e Zieschang [12] considerando uma distribuição logística dependendo da variável resposta $Y$ e das variáveis $Z$ do processo de amostragem considerando o modelo populacional $f_{p}\left(y_{i} \mid \boldsymbol{x}_{i}, \boldsymbol{\theta}\right)$ com distribuição normal. Nesse trabalho, a função de verossimilhança foi construída considerando os elementos da amostra respondentes e os não-respondentes utilizando máxima verossimilhança para estimar os parâmetros do modelo. Em seguida, o valor esperado para os elementos não-respondentes foi estimado utilizando técnicas numéricas de integração.

Posteriormente, Beaumont [3] também considerou a modelagem da probabilidade de não-resposta por uma função logística considerando o caso em que os resíduos do modelo populacional $f_{p}\left(y_{i} \mid \boldsymbol{x}_{i}, \boldsymbol{\theta}\right)$ tem distribuição normal.

Por outro lado, o trabalho de Tang, Little e Raghunathan [41] não considera a modelagem da probabilidade de não-resposta argumentando que na prática o mecanismo de dados faltantes não é bem entendido e que a forma funcional da probabilidade de não-resposta é desconhecida. Nesse trabalho, a estimação dos parâmetros dos modelo foi realizada considerado uma pseudoverossimilhança baseada na distribuição das covariáveis do problema.

Diferentemente dos trabalhos de Greenlees, Reece e Zieschang [12], Beaumont [3] e Tang, Little e Raghunathan [41] citados anteriomente, ao utilizar as distribuições (2.20) e (2.21) não é necessário conhecer os valores das covariáveis nas unidades não-respondentes 
e nem modelar a distribuição das covariáveis amostradas.

É importante enfatizar que nos casos de amostragem informativa, a distribuição amostral dada por (2.13) refere-se à distribuição das medidas amostrais, definidas pelo modelo populacional e o processo de seleção da amostra, considerando a amostra de entrevistados fixa. Isso implica, por exemplo, que os conglomerados amostrados em dois estágios e as covariáveis observadas $\left\{\boldsymbol{x}_{i}, i \in S\right\}$ estão fixadas em relação ao modelo. O mesmo é válido para o caso das unidades respondentes, que dependem também do processo de não-resposta. Por outro lado, a distribuição aleatorizada dos dados, condicionada aos valores populacionais $\left\{y_{i}, \boldsymbol{x}_{i}, i \in U\right\}$, que são tratados como valores fixos, e os elementos estocásticos usados na inferência referem-se a seleção aleatória da amostra (ou dos respondentes). A utilização dessa distribuição não está condicionada aos conglomerados amostrados ou às covariáveis observadas.

\subsection{Relações gerais de invariância}

Na presente Seção são apresentadas condições gerais, propostas por Pfeffermann, Krieger e Rinott [28], sob as quais a distribuição amostral pertence à mesma família da distribuição populacional.

Para isso, considere que a função de densidade de probabilidade populacional pertence a família exponencial, isto é,

$$
f_{p}\left(y_{i} \mid x_{i}, \boldsymbol{\theta}_{i}\right)=a_{i}\left(\boldsymbol{\theta}_{i}\right) \exp \left[\sum_{k=1}^{K} \theta_{k i} b_{k i}\left(y_{i}\right)+c_{i}\left(y_{i}\right)\right]
$$

onde $\boldsymbol{\theta}_{i}=\left(\theta_{1 i}, \ldots, \theta_{K i}\right)^{\prime}$ define a parametrização natural da família com valores no espaço de parâmetros $\Theta \subset \mathrm{R}^{K} \operatorname{com} b_{k i}($.$) e c_{k i}($.$) sendo funções conhecidas.$

Além disso, suponha que as probabilidades de inclusão da amostra tenham valor esperado

$$
E_{p}\left(\pi_{i} \mid y_{i}, x_{i}, \boldsymbol{\psi}_{i}\right)=r_{i} \exp \left[\sum_{k=1}^{K} \psi_{k i} b_{k i}\left(y_{i}\right)\right],
$$

onde os $r_{i}$ 's são constantes que podem depender de $x_{i}$, mas não de $y_{i}$, e os $\left\{\psi_{k i}\right\}$ são os parâmetros do processo de amostragem. 
Proposição 2.5.1. Se a função de densidade de probabilidade populacional de $Y_{i}$ pertence a familia exponencial definida por (2.22) e a probabilidade de inclusão da amostra satisfaz (2.23), então a função de densidade de probabilidade amostral de $y_{i}$ pertence a mesma família exponencial com parâmetros $\theta_{k i}^{*}=\theta_{k i}+\psi_{k i}\left(\right.$ com a condição que $\left.\theta_{i k}^{*} \in \Theta\right)$.

O resultado estabelecido na Proposição 2.5.1 é semelhante a uma questão familiar de identificação das distribuições a priori em inferência bayesiana. Cox e Hinkley [7] considera a família de distribuições a priori na qual a distribuição a posteriori pertence a mesma família como invariante sob amostragem. No presente contexto, esta terminologia é perfeitamente adequada.

A dependência de $\boldsymbol{x}_{i}$ nas equações (2.22) e (2.23) ocorre geralmente por meio de $\theta_{k i} \mathrm{e}$ $\psi_{k i}$ respectivamente. Esta dependência pode ocorrer mais explicitamente para uma classe de modelos de regressão de $Y$ em $X$ se as seguintes relações são consideradas verdadeiras

$$
\theta_{k i}=\phi_{0 k}+\boldsymbol{x}_{i}^{\prime} \phi_{k} \text { e } \psi_{k i}=\gamma_{0 k}+\boldsymbol{x}_{i}^{\prime} \gamma_{k}
$$

Corolário 2.5.1. Considerando válidas as condições do Proposição 2.5 .1 e as igualdades em (2.24), a função de densidade de probabilidade amostral pertence a mesma família com parâmetros $\theta_{i k}=\left(\phi_{0 k}+\gamma_{0 k}\right)+\boldsymbol{x}_{i}^{\prime}\left(\boldsymbol{\phi}_{k}+\boldsymbol{\gamma}_{k}\right)$. Em particular, se as funções $\psi_{k i}$ não dependem de $\boldsymbol{x}_{i}$, isto é $\boldsymbol{\gamma}_{k}=\mathbf{0}$, os coeficientes de $\boldsymbol{x}_{i}$ na parametrização natural da função de densidade de probabilidade amostral são os mesmos da função de densidade de probabilidade populacional.

\subsection{Distribuições amostrais em projetos amostrais ge- rais}

$\mathrm{Na}$ presente Seção, considere conhecida a forma da esperança condicional $E_{p}\left(\pi_{i} \mid y_{i}, \boldsymbol{x}_{i}, \boldsymbol{\psi}\right)$. Obviamente isso pode não ser verdade na prática, particularmente quando os $\pi_{i}$ 's dependem também de variáveis utilizadas no projeto amostral que não estão contidas entre as variáveis concomitantes $X$.

Uma vantagem importante da utilização de probabilidade amostral é que, exceto em casos de não-resposta, as probabilidades de seleção da amostra são sempre conhecidas. 
Considerando que elas estão disponíveis para o analista pelo menos nas unidades da amostra, as esperanças condicionais $E_{p}\left(\pi_{i} \mid y_{i}, \boldsymbol{x}_{i}, \boldsymbol{\psi}\right)$ podem ser estimadas por meio dos dados amostrais.

\subsubsection{Seleção com probabilidade proporcional ao tamanho}

Suponha que os $\pi_{i}$ 's são medidas de tamanho, consideradas medidas de alguma função de densidade de probabilidade $g(\pi)$. Sob algumas condições de regularidade, a esperança $E_{p}\left(\pi_{i} \mid y_{i}, \boldsymbol{x}_{i}, \boldsymbol{\psi}\right)$ pode ser aproximada por polinômios de baixa ordem em função de $y_{i}$, das componentes de $\boldsymbol{x}_{i}$ e dos parâmetros $\boldsymbol{\eta}^{T}=\left(\eta_{0}, \ldots, \eta_{d}\right)$ e $\boldsymbol{\lambda}^{T}=\left(\lambda_{11}, \ldots, \lambda_{l(p) p}\right)$, ou por exponenciais de tais polinômios, por meio das séries de Taylor.

Para o primeiro caso, considere $\boldsymbol{x}_{i}=\left(x_{i 1}, x_{i 2}, \ldots, x_{i m}\right)$. Assim,

$$
E_{p}\left(\pi_{i} \mid y_{i}, \boldsymbol{x}_{i}, \boldsymbol{\eta}, \boldsymbol{\lambda}\right) \approx \sum_{j=0}^{d} \eta_{j} y_{i}^{j}+\sum_{p=1}^{m} h\left(x_{i p} \mid \boldsymbol{\lambda}\right)
$$

onde $d$ é o grau do polinômio em $y_{i}$ e $h$ é um polinômio em função de $x_{i p} \mid \boldsymbol{\lambda}$ dado por

$$
h\left(x_{i p} \mid \boldsymbol{\lambda}\right)=\sum_{k=1}^{l(p)} \lambda_{k p} x_{i p}^{k}=\lambda_{1 p} x_{i p}+\lambda_{2 p} x_{i p}^{2}+\lambda_{3 p} x_{i p}^{3}+\cdots+\lambda_{l(p) p} x_{i p}^{l(p)}
$$

com $l(p)$ igual ao grau do polinômio em $x_{i p}$ e $\left\{\eta_{j}\right\}$ e $\left\{\lambda_{k p}\right\}$ são parâmetros desconhecidos do mecanismo de seleção da amostra.

Substituindo (2.25) em (2.13) e supondo a existência dos momentos $E^{(j)}=E_{p}\left(Y_{i}^{j} \mid \boldsymbol{x}_{i}, \boldsymbol{\theta}\right)$, a função de densidade de probabilidade amostral pode ser aproximada por

$$
f_{s}\left(y_{i} \mid \boldsymbol{x}_{i}, \boldsymbol{\theta}, \boldsymbol{\eta}, \boldsymbol{\lambda}\right) \approx \frac{\left[\sum_{j=0}^{d} \eta_{j} y_{i}^{j}+\sum_{p=1}^{m} h\left(x_{i p} \mid \boldsymbol{\lambda}\right)\right] f_{p}\left(y_{i} \mid \boldsymbol{x}_{i}, \boldsymbol{\theta}\right)}{\eta_{0}+\sum_{j=1}^{d} \eta_{j} E_{p}\left(y_{i}^{j} \mid \boldsymbol{x}_{i}, \boldsymbol{\theta}\right)+\sum_{p=1}^{m} h\left(x_{i p} \mid \boldsymbol{\lambda}\right)} .
$$

Como a distribuição definida em (2.27) depende dos parâmetros do processo amostral que geralmente são desconhecidos, o processo de inferência deve considerar a estimação desses parâmetros. Para isso, a distribuição amostral de $z_{i} \mid y_{i}, x_{i}, \boldsymbol{\eta}, \boldsymbol{\lambda}$ deve ser incluída no modelo. Assim, como $\pi_{i}=\operatorname{Pr}\left(I_{i}=1 \mid Z_{U}\right)=C z_{i}$, onde $C=\frac{n}{N \bar{z}}$ é constante, tem-se 
que os valores esperados de $\pi_{i}$ e a distribuição amostral de $z_{i} \mid y_{i}, \boldsymbol{x}_{i}, \boldsymbol{\eta}, \boldsymbol{\lambda}$ são dados por

$$
\begin{aligned}
E_{p}\left(\pi_{i} \mid z_{i}, y_{i}, \boldsymbol{x}_{i}, \boldsymbol{\eta}, \boldsymbol{\lambda}\right) & =C E_{p}\left(z_{i} \mid z_{i}, y_{i}, \boldsymbol{x}_{i}, \boldsymbol{\eta}, \boldsymbol{\lambda}\right)=C z_{i}, \\
E_{p}\left(\pi_{i} \mid y_{i}, \boldsymbol{x}_{i}, \boldsymbol{\eta}, \boldsymbol{\lambda}\right) & =C \int z_{i} f_{p}\left(z_{i} \mid y_{i}, \boldsymbol{x}_{i}, \boldsymbol{\eta}, \boldsymbol{\lambda}\right) d z_{i} \\
& =C\left[\sum_{j=0}^{d} \eta_{j} y_{i}^{j}+\sum_{p=1}^{m} h\left(x_{i p} \mid \boldsymbol{\lambda}\right)\right], \\
f_{s}\left(z_{i} \mid y_{i}, \boldsymbol{x}_{i}, \boldsymbol{\eta}, \boldsymbol{\lambda}\right) & =\frac{E_{p}\left(\pi_{i} \mid z_{i}, y_{i}, \boldsymbol{x}_{i}, \boldsymbol{\eta}, \boldsymbol{\lambda}\right) f_{p}\left(z_{i}, y_{i}, \boldsymbol{x}_{i}, \boldsymbol{\eta}, \boldsymbol{\lambda}\right)}{E_{p}\left(\pi_{i} \mid y_{i}, \boldsymbol{x}_{i}, \boldsymbol{\eta}, \boldsymbol{\lambda}\right)} \\
& =\frac{z_{i} f_{p}\left(z_{i} \mid y_{i}, \boldsymbol{x}_{i}, \boldsymbol{\eta}, \boldsymbol{\lambda}\right)}{\sum_{j=0}^{d} \eta_{j} y_{i}^{j}+\sum_{p=1}^{m} h\left(x_{i p} \mid \boldsymbol{\lambda}\right)} .
\end{aligned}
$$

Consequentemente, considerando $\boldsymbol{\psi}^{T}=\left(\eta_{0}, \ldots, \eta_{d}, \lambda_{11}, \ldots, \lambda_{l(m) m}\right)$ os parâmetros do processo de amostragem, $\boldsymbol{\theta}$ os parâmetros da distribuição populacional e a distribuição $a$ priori $f(\boldsymbol{\theta}, \boldsymbol{\psi})$, a distribuição conjunta a posteriori é dada por

$$
f(\boldsymbol{\theta}, \boldsymbol{\psi} \mid \boldsymbol{y}, X, Z) \propto f(\boldsymbol{\theta}, \boldsymbol{\psi}) \prod_{i=1}^{n} f_{s}\left(y_{i} \mid \boldsymbol{x}_{i}, \boldsymbol{\theta}, \boldsymbol{\psi}\right) \prod_{i=1}^{n} f_{s}\left(z_{i} \mid y_{i}, \boldsymbol{x}_{i}, \boldsymbol{\psi}\right) .
$$

Nesse caso, o vetor de parâmetros $(\boldsymbol{\theta}, \boldsymbol{\psi})$ da função de densidade de probabilidade amostral $f_{s}\left(y_{i} \mid \boldsymbol{x}_{i}, \boldsymbol{\theta}, \boldsymbol{\psi}\right)$ é formado pelo vetor de parâmetros $\boldsymbol{\theta}$ da função de densidade de probabilidade populacional $f_{p}\left(y_{i} \mid \boldsymbol{x}_{i}, \boldsymbol{\theta}\right)$ e pelo vetor de parâmetros $\boldsymbol{\psi}$ do processo de seleção da amostra. Logo, a função de densidade de probabilidade amostral pode depender de mais parâmetros do que a função de densidade de probabilidade populacional.

Por outro lado, considere a aproximação

$$
E_{p}\left(\pi_{i} \mid y_{i}, \boldsymbol{x}_{i}, \boldsymbol{\eta}, \boldsymbol{\lambda}\right) \approx \exp \left[\sum_{j=0}^{d} \eta_{j} y_{i}^{j}+\sum_{p=1}^{m} h\left(x_{i p} \mid \boldsymbol{\lambda}\right)\right],
$$

onde $d$ é novamente o grau do polinômio definido em $y_{i}$ e os polinômios $h\left(x_{i p} \mid \boldsymbol{\lambda}\right)$ estão definidos em (2.26).

Essa situação é mais frequente quando a seleção da amostra é realizada em vários estágios, tais que as probabilidades de inclusão final são o produto das probabilidades de seleção em todos os estágios. Se o vetor $\boldsymbol{x}_{i}$ contém as variáveis de seleção da amostra 
utilizadas nos vários estágios, é natural expressar as esperanças $E_{p}\left(\pi_{i} \mid y_{i}, \boldsymbol{x}_{i}, \boldsymbol{\eta}, \boldsymbol{\lambda}\right)$ como a função multiplicativa de $y_{i}$ e $\boldsymbol{x}_{i}$. Assim,

$$
f_{s}\left(y_{i} \mid \boldsymbol{x}_{i}, \boldsymbol{\theta}, \boldsymbol{\eta}, \boldsymbol{\lambda}\right) \approx \frac{\exp \left[\sum_{j=0}^{d} \eta_{j} y_{i}^{j}+\sum_{p=1}^{m} h\left(x_{i p} \mid \boldsymbol{\lambda}\right)\right] f_{p}\left(y_{i} \mid \boldsymbol{x}_{i}, \boldsymbol{\theta}\right)}{E_{p}\left[\exp \left(\sum_{j=0}^{d} \eta_{j} y_{i}^{j}+\sum_{p=1}^{m} h\left(x_{i p} \mid \boldsymbol{\lambda}\right)\right) \mid \boldsymbol{x}_{i}\right]}
$$

A desvantagem da aproximação (2.32) e da expressão resultante (2.33) é que alguns dos parâmetros da função de densidade de probabilidade populacional não podem ser identificados pelas observações amostrais de $Y$ e $X$ sozinhas. Por exemplo, no caso da regressão linear do exemplo 2.3.1, o intercepto $\beta_{0}$ da regressão populacional não pode ser separado do intercepto $\left(\beta_{0}+\alpha_{1} \sigma^{2}\right)$ da regressão amostral a menos que o coeficiente amostral $\alpha_{1}$ seja estimado separadamente.

Quando o número de parâmetros da função de densidade de probabilidade amostral é muito grande, é mais fácil estimar esse parâmetros em dois passos. No primeiro passo, os coeficientes $\left\{\eta_{j}\right\}$ e $\left\{\lambda_{k p}\right\}$ são estimados das probabilidades observadas $\pi_{i}$, utilizando as relações (2.25) e (2.32). No segundo passo, os parâmetros da função de densidade de probabilidade populacional são estimados pela máxima verossimilhança ou por qualquer outro método utilizando as estimativas de $\left\{\eta_{j}\right\}$ e $\left\{\lambda_{k p}\right\}$ encontradas no primeiro passo.

\subsubsection{Seleção com amostragem estratificada}

Na presente seção, considera-se probabilidades de seleção constantes dentro de cada estrato. Com isso, $E_{p}\left(\pi_{i} \mid y_{i}, \boldsymbol{x}_{i}, \boldsymbol{\psi}\right)$ não pode ser considerada contínua em y e $\boldsymbol{x}$ para a utilização de aproximações por séries de Taylor, como no caso de amostragem com probabilidade proporcional ao tamanho.

Seja $Z_{i}=q\left(Y_{i}, \boldsymbol{x}_{i}, \boldsymbol{\psi}\right)$ uma variável aleatória com $\boldsymbol{x}_{i}$ podendo ser fixo ou aleatório e $\boldsymbol{\psi}$ uma vetor de parâmetros. Considere $a^{(0)}<a^{(1)}<\cdots<a^{(L)}$ os $L$ percentiles da distribuição $Z \operatorname{com} a^{(0)}=-\infty$ e $a^{(L)}=\infty$. Esses percentiles definem uma divisão dos valores populacionais em $L$ estratos $U_{1}, U_{2}, \ldots, U_{L}$ de tamanhos $N_{1}, N_{2}, \ldots, N_{L}$ baseados nos valores realizados $z_{1}, z_{2}, \ldots, z_{N}$. A divisão é tal que a unidade $i$ pertence ao estrato $U_{l}$ se e somente se $a^{(l-1)}<z_{i}<a^{(l)}$, definindo assim a variável $\boldsymbol{O}$ tal que $O_{i}=l$ indica 
que a unidade $i$ pertence ao estrato $l$, isto é,

$$
O_{i}=\left\{\begin{array}{cl}
1, & z_{i}<a^{(1)} \\
2, & a^{(1)} \leq z_{i}<a^{(2)} \\
\vdots & \\
i, & a^{(i-1)} \leq z_{i}<a^{(i)} \\
\vdots & \\
L, & z_{i} \geq a^{(L-1)}
\end{array}\right.
$$

Supondo amostragem estratificada desproporcional, a amostragem é informativa pois a divisão dos estratos está relacionada com a variável resposta. Considerando os tamanhos amostrais $n_{l}$ e $P_{l}=n_{l} / N_{l}$ as proporções amostradas em cada estrato $l=1, \ldots, L$, tem-se

$$
\begin{aligned}
E_{p}\left(\pi_{i} \mid y_{i}, \boldsymbol{x}_{i}, \boldsymbol{\psi}\right) & =\operatorname{Pr}\left(i \in S \mid y_{i}, \boldsymbol{x}_{i}, \boldsymbol{\psi}\right) \\
& =\sum_{k=1}^{L} \operatorname{P} \operatorname{Pr}\left(O_{i}=k \mid y_{i}, \boldsymbol{x}_{i}, \boldsymbol{\psi}\right) \\
& =\sum_{k=1}^{L} \operatorname{P}_{k} \operatorname{Pr}\left(a^{(k-1)} \leq z_{i}<a^{(k)} \mid y_{i}, \boldsymbol{x}_{i}, \boldsymbol{\psi}\right) .
\end{aligned}
$$

Analogamente, tem-se

$$
\begin{aligned}
E_{p}\left(\pi_{i} \mid \boldsymbol{x}_{i}, \boldsymbol{\psi}\right) & =\operatorname{Pr}\left(i \in S \mid \boldsymbol{x}_{i}, \boldsymbol{\psi}\right) \\
& =\sum_{k=1}^{L} \operatorname{P}_{k} \operatorname{Pr}\left(O_{i}=k \mid \boldsymbol{x}_{i}, \boldsymbol{\psi}\right) \\
& =\sum_{k=1}^{L} \operatorname{P}_{k} \operatorname{Pr}\left(a^{(k-1)} \leq z_{i}<a^{(k)} \mid \boldsymbol{x}_{i}, \boldsymbol{\psi}\right) \\
& =\sum_{k=1}^{L}\left[P_{k} \int_{a^{(k-1)}}^{a^{(k)}} f_{p}\left(z \mid \boldsymbol{x}_{i}, \boldsymbol{\psi}\right) d z\right]
\end{aligned}
$$

Assim, a função de densidade de probabilidade amostral de $Y_{i} \mid \boldsymbol{x}_{i}$ é dada por

$$
f_{s}\left(y_{i} \mid \boldsymbol{x}_{i}, \boldsymbol{\theta}, \boldsymbol{\psi}\right)=\left\{\begin{array}{cl}
P_{1} f_{p}\left(y_{i} \mid \boldsymbol{x}_{i}, \boldsymbol{\theta}\right) / E_{p}\left(\pi_{i} \mid \boldsymbol{x}_{i}, \boldsymbol{\psi}\right) & \text { se } z_{i} \leq a^{(1)} \\
P_{2} f_{p}\left(y_{i} \mid \boldsymbol{x}_{i}, \boldsymbol{\theta}\right) / E_{p}\left(\pi_{i} \mid \boldsymbol{x}_{i}, \boldsymbol{\psi}\right) & \text { se } a^{(1)}<z_{i} \leq a^{(2)} \\
\vdots & \\
P_{L} f_{p}\left(y_{i} \mid \boldsymbol{x}_{i}, \boldsymbol{\theta}\right) / E_{p}\left(\pi_{i} \mid \boldsymbol{x}_{i}, \boldsymbol{\psi}\right) & \text { se } a^{(L-1)}<z_{i} .
\end{array}\right.
$$


Similarmente, a distribuição amostral de $z_{i} \mid y_{i}$ pode ser definida por

$$
f_{s}\left(z_{i} \mid y_{i}, \boldsymbol{\psi}\right)=\frac{E_{p}\left(\pi_{i} \mid z_{i}, y_{i}, \boldsymbol{\psi}\right) f_{p}\left(z_{i} \mid y_{i}, \boldsymbol{\psi}\right)}{E_{p}\left(\pi_{i} \mid y_{i}, \boldsymbol{\psi}\right)}
$$

onde

$$
E_{p}\left(\pi_{i} \mid z_{i}, y_{i}, \boldsymbol{\psi}\right)=\operatorname{Pr}\left(i \in S \mid z_{i}, y_{i}, \boldsymbol{\psi}\right)=\operatorname{Pr}\left(i \in S \mid \boldsymbol{\psi}, O_{i}=k\right)=P_{k},
$$

e $E_{p}\left(\pi_{i} \mid y_{i}, \boldsymbol{\psi}\right)=E_{p}\left(\pi_{i} \mid y_{i}, \boldsymbol{x}_{i}, \boldsymbol{\psi}\right)$ dado por (2.35) pois

$$
\operatorname{Pr}\left(a^{(k-1)}<z_{i} \leq a^{(k)} \mid y_{i}, \boldsymbol{x}_{i}, \boldsymbol{\psi}\right)=\operatorname{Pr}\left(a^{(k-1)}<z_{i} \leq a^{(k)} \mid y_{i}, \boldsymbol{\psi}\right) .
$$




\section{CAPÍtulo 3}

\section{FBST}

Testes de significância para hipóteses precisas são procedimentos frequentemente utilizados para medir a consistência dos dados com alguma hipótese precisa. Esses procedimentos compõem um problema antigo e controverso na inferência estatística, pois tanta a escola bayesiana quanto a frequentista têm apresentado frequentemente soluções para esse problema sem considerar questões fundamentais tais como a medida da hipótese precisa.

O objetivo do presente Capítulo é apresentar uma medida bayesiana coerente para o valor de evidência para hipóteses precisas e um teste de significância baseado neste valor de evidência chamado FBST (Full Bayesian Significance Test), proposto por Pereira e Stern [24]. Esse método foi apresentado considerando que testes de significância para hipóteses precisas precisam ser realizados.

O FBST é intuitivo, tem uma caracterização geométrica e pode ser implementado utilizando modernas técnicas de otimização e integração numérica. Além disso, o método é completamente bayesiano, pois é necessário conhecer somente o espaço paramétrico representado pela função de densidade a posteriori, e consiste na análise de conjuntos de credibilidade.

O FBST tem sido aplicado com sucesso em inúmeros problemas estatísticos relevantes, tais como: teste de homogeneidade e independência em tabela de contingência; comparação de coeficientes de variação (veja Pereira e Stern [25]); problema de Behrens-Fisher multivariado; teste de equilíbrio de Hardy-Weinberg; seleção de variáveis; teste de independência da distribuição de Holgate (Poisson bivariada) (veja Stern e Zacks [39]) e teste de Weibull (veja Irony et al. [14]) 


\subsection{Definição intuitiva do FBST}

Na presente Seção é apresentada a versão intuitiva do FBST introduzida por Pereira e Stern [24] juntamente com um exemplo de um problema de inferência o qual este teste já foi utilizado anteriormente.

Para isso, considere um espaço estatístico onde $\Theta \subset \mathrm{R}^{m}$ é o espaço paramétrico, $\chi \subset \mathrm{R}^{k}$ é o espaço amostral, $\theta \in \Theta$ é o parâmetro, $f(\theta)$ é a função de densidade a priori sobre $\Theta$, $\boldsymbol{x}$ é um vetor com os dados observados e $L_{x}(\theta)$ é a verossimilhança gerada pelos dados $\boldsymbol{x}$.

Definição 3.1.1. Uma hipótese precisa $H$ estabelece que $\theta$ pertence a uma subvariedade $\Theta_{H}$ de dimensão menor que $\Theta$.

A hipótese $H$ é precisa quando o subconjunto $\Theta_{H}$ tem medida de Lebesgue nula. Na construção do FBST todos os conjuntos de mesma natureza são tratados da mesma maneira e, como consequência disso, os conjuntos que definem hipóteses precisas têm sempre medida nula. Nesta construção, ao invés de mudar a natureza do $H$ supondo probabilidade positiva nesse conjunto, trabalha-se com o conjunto tangente $T$ dos pontos que têm os valores da função densidade de probabilidade a posteriori maiores que qualquer valor dessa função restrita ao conjunto $\Theta_{H}$.

Após a observação dos dados $\boldsymbol{x}$, o único ente relevante para a avaliação do valor de evidência bayesiano a favor de $H$, ev, é a função densidade de probabilidade a posteriori de $\theta$ dado $\boldsymbol{x}$, denotada por

$$
f_{n}(\theta \mid \boldsymbol{x}) \propto f(\theta) L_{x}(\theta)
$$

Definição 3.1.2. Considere uma hipótese precisa $H: \theta \in \Theta_{H}$,

$$
f^{*}=\sup _{\theta \in \Theta_{H}} f_{n}(\theta \mid \boldsymbol{x}) \text { e } T=\left\{\theta \in \Theta \mid f_{n}(\theta \mid \boldsymbol{x})>f^{*}\right\} .
$$

O valor de evidência bayesiano contra $H$, considerando os dados amostrais $\boldsymbol{x}$ conhecidos, é definido pela probabilidade a posteriori do conjunto tangente, isto é,

$$
\overline{e v}=\operatorname{Pr}(\theta \in T \mid \boldsymbol{x})=\int_{T} f_{n}(\theta \mid \boldsymbol{x}) d \theta .
$$


Observe que o valor de evidência a favor de $H$, ev $=1-\overline{e v}$, não é uma evidência contra a hipótese alternativa $\bar{H}$ (que não é precisa). Equivalentemente, ev não é evidência a favor de $\bar{H}$ embora seja contra $H$.

Definição 3.1.3. O FBST (Full Bayesian Significance Test) é um procedimento que rejeita a hipótese precisa $H$ quando ev é pequeno.

Para ilustrar a utilização do FBST, segue um exemplo de um problema de inferência trabalhado primeiramente por McNemar [21] e posteriormente discutido por Irony, Pereira e Tiwari [13] e por Pereira, Stern e Wechsler [26].

Exemplo 3.1.1. Dois professores, Ed e Joe, do Departamento de Odontologia avaliaram a habilidade de 224 estudantes em fazer obturações dentais. Cada estudante foi avaliado pelos dois professores e o resultado da avaliação deve ser aprovado (A) ou reprovado ( $R$ ), pois o departamento quer avaliar se os professores são igualmente exigentes. A tabela abaixo apresenta os resultados.

\begin{tabular}{|c|c|c|c|c|}
\cline { 3 - 5 } \multicolumn{2}{c|}{} & \multicolumn{3}{|c|}{ Joe } \\
\cline { 3 - 5 } Ed & $A$ & $R$ & Total \\
\hline \multirow{3}{*}{$E$} & $A$ & 62 & 41 & 103 \\
\cline { 2 - 5 } & $R$ & 25 & 96 & 121 \\
\cline { 2 - 5 } & Total & 87 & 137 & 224 \\
\hline
\end{tabular}

Nesse exemplo, tem-se quatro classes de classificação com probabilidades $\theta_{11}, \theta_{12}, \theta_{21} e$ $\theta_{22}$. A hipótese $H$ a ser testada é a chance dos dois professores reprovarem (ou aprovarem) a mesma quantidade de alunos. Assim, $H: \theta_{1, \bullet}=\theta_{2, \bullet}$ que é equivalente âA $H: \theta_{12}=\theta_{21}$ (contra $\bar{H}: \theta_{12} \neq \theta_{21}$ ).

Considerando uma distribuição a priori uniforme, isto é, $f(\theta)=\operatorname{Dirichlet}(1,1,1,1)$, a função de densidade a posteriori é $f_{n}(\theta \mid \boldsymbol{x})=\operatorname{Dirichlet}(63,42,26,97)$.

O primeiro passo para calcular o valor de evidência é obter um valor $f^{*}=f_{n}\left(\theta^{*} \mid \boldsymbol{x}\right)$, satisfazendo a hipótese $H$, que maximize a função de densidade a posteriori

$$
f_{n}\left(\theta_{11}, \theta_{12}, \theta_{21}, \theta_{22} \mid \boldsymbol{x}\right)=\frac{\Gamma(224)}{\Gamma(62) \Gamma(41) \Gamma(25) \Gamma(96)} \theta_{11}{ }^{62} \theta_{12}{ }^{41} \theta_{21}{ }^{25} \theta_{22}{ }^{96} .
$$


Assim, deseja-se resolver o seguinte problema de otimização com restrições de igualdade

$$
\begin{array}{ll}
\text { Maximizar } & f_{n}\left(\theta_{11}, \theta_{12}, \theta_{21}, \theta_{22} \mid \boldsymbol{x}\right) \\
\text { Sujeito a } & \theta_{12}=\theta_{21} \\
& \theta_{11}+\theta_{12}+\theta_{21}+\theta_{22}=1
\end{array}
$$

Resolver esse problema com restrições é equivalente a resolver o seguinte problema de otimização sem restrições

$$
\text { Maximizar } g_{n}\left(\theta_{12}, \theta_{22} \mid \boldsymbol{x}\right) \propto\left(1-2 \theta_{12}-\theta_{22}\right)^{62} \theta_{12}{ }^{66} \theta_{22}{ }^{96}
$$

cuja solução é $\theta_{12}^{*}=33 / 224$ e $\theta_{22}^{*}=96 / 224$.

Portanto, a distribuição a posteriori atinge valor máximo condicionada a hipótese $H$ no ponto $\theta^{*}=\frac{1}{224}(62,33,33,96)$ e o valor máximo obtido é 622 .

Com isso, o conjunto tangente é definido por $T=\left\{\theta \in \Theta ; f_{n}(\theta \mid \boldsymbol{x})>622\right\}$, onde $\Theta=\left\{\theta=\left(\theta_{11}, \theta_{12}, \theta_{21}, \theta_{22}\right) ; \theta_{11}+\theta_{12}+\theta_{21}+\theta_{22}=1\right.$ e $\left.\theta_{i j}>0\right\}$.

Finalmente, integrando numericamente obtem-se ev $=0,2641$.

\subsection{Definição invariante do FBST}

Na presente Seção, apresenta-se uma versão invariante do FBST sob transformações no sistema de coordenadas no espaço de parâmetros proposta por Madruga, Pereira e Stern [20], que é uma generalização da Definição 3.1.2 de valor de evidência apresentada na Seção anterior. Para isso, considere $r(\theta)$ uma função densidade de referência em $\Theta$.

Definição 3.2.1. A função $s_{n}(\theta \mid \boldsymbol{x})=f_{n}(\theta \mid \boldsymbol{x}) / r(\theta)$ é chamada de surpresa relativa a posteriori para uma dada função densidade de probabilidade $r(\theta)$ definida em $\Theta$.

Definição 3.2.2. Considere uma hipótese precisa $H: \theta \in \Theta_{H}$,

$$
s^{*}=\sup _{\theta \in \Theta_{H}} s_{n}(\theta \mid \boldsymbol{x}) \text { e } T=\left\{\theta \in \Theta \mid s_{n}(\theta \mid \boldsymbol{x})>s^{*}\right\} .
$$

O valor de evidência invariante contra a hipótese $H$, considerando os dados amostrais $\boldsymbol{x}$ conhecidos, é definida por

$$
\overline{e v}=\int_{T} f_{n}(\theta \mid \boldsymbol{x}) d \theta .
$$


O conjunto $T$ formado pelos pontos $\theta \in \Theta$ com surpresa relativa maior que qualquer ponto de $\Theta_{H}$ é chamado conjunto de surpresa relativa mais alta (highest relative surprise set - HRSS).

Definição 3.2.3. A versão invariante do FBST é um procedimento que rejeita a hipótese precisa $H$ quando ev $=1-\overline{e v}$ é pequeno.

Proposição 3.2.1. A Definição 3.2.2 de valor de evidência contra $H$ é invariante com respeito âA reparametrizações das coordenadas de $\theta$.

Prova. Considere $\phi$ é uma função bijetora, continuamente derivável e integrável tal que $\omega=\phi(\theta)$ é uma reparametrização de $\theta$ e $J(\omega)$ é a matriz Jacobiana dessa transformação, isto é,

$$
J(\omega)=\left[\frac{\partial}{\partial w} \Phi^{-1}(\omega)\right]=\left[\frac{\partial \theta}{\partial \omega}\right]=\left[\begin{array}{ccc}
\frac{\partial \theta_{1}}{\partial \omega_{1}} & \cdots & \frac{\partial \theta_{1}}{\partial \omega_{n}} \\
\vdots & \ddots & \vdots \\
\frac{\partial \theta_{n}}{\partial \omega_{1}} & \cdots & \frac{\partial \theta_{n}}{\partial \omega_{n}}
\end{array}\right] .
$$

Como a função de densidade a posteriori de $\omega$, dado $\boldsymbol{x}$, pode ser escrita como

$$
\tilde{f}_{n}(\omega)=f_{n}\left(\phi^{-1}(\omega)\right)|J(\omega)|
$$

e a função densidade de referência após a reparametrização é

$$
\tilde{r}(\omega)=r\left(\phi^{-1}(\omega)\right)|J(\omega)|,
$$

então a função surpresa relativa é

$$
\tilde{s}_{n}(\omega)=\frac{\tilde{f}_{n}(\omega)}{\tilde{r}(\omega)}=\frac{f_{n}\left(\phi^{-1}(\omega)\right)}{r\left(\phi^{-1}(\omega)\right)} .
$$

Considere $\Omega_{H}=\phi\left(\Theta_{H}\right)$, tem-se

$$
\tilde{s}^{*}=\sup _{\omega \in \Omega_{H}} \tilde{s}_{n}(\omega)=\sup _{\theta \in \Theta_{H}} s_{n}(\theta)=s^{*} .
$$

Portanto, $\phi\left(T_{\Theta}\right)=T_{\Omega}$ e o valor de evidência sob parametrização é

$$
1-\tilde{e} v=\int_{T_{\Omega}} \tilde{f}_{n}(\omega) d \omega=\int_{T_{\Theta}} f_{n}(\theta) d \theta=1-e v=\overline{e v} .
$$

Note que a definição invariante do FBST está de acordo com a definição intuitiva do FBST quando a densidade de referência é a densidade uniforme. 


\subsection{Função de perda para o FBST}

Na presente Seção, são apresentadas funções de perdas para teste de significância para hipóteses precisas utilizando o FSBT. Para isso será considerado somente a função densidade de probabilidade a posteriori sobre $\Theta$, sem a necessidade de introduzir uma probabilidade positiva para $\Theta_{H}$ como no teste de Jeffrey. Essas funções de perdas foram propostas por Madruga, Esteves e Wechsler [19] e estão em harmonia com a teoria bayesiana de decisão de Rubin [37].

Considere a hipótese precisa $H: \theta=\theta_{0}$ contra a hipótese $\bar{H}: \theta \neq \theta_{0}$ e o espaço de decisão $D=\left\{\right.$ Aceitar $H\left(d_{0}\right)$, Não Aceitar $\left.H\left(d_{1}\right)\right\}$.

Definição 3.3.1. A função de perda $L$ em $D \times \Theta$ definida por $L(N \tilde{a} o$ Aceitar $H, \theta)=a[1-I(\theta \in T(\boldsymbol{x}))]$ e $L($ Aceitar $H, \theta)=b+c I(\theta \in T(\boldsymbol{x}))$, com $a, b$ e c constantes positivas, é chamada função de perda $L P_{1}$.

Um aspecto importante das funções de perda $L P_{1}$ é que elas punem pesadamente quem aceita a hipótese precisa $H$ quando $\theta$ é mais provável que $\theta_{0}$, isto é, quando $\theta$ pertence a $T(\boldsymbol{x})$.

Teorema 3.3.1. A minimização da esperança a posteriori da função de perda $L P_{1}$ é um procedimendo equivalente ao FBST.

Prova. O risco a posteriori de aceitação é

$$
\begin{aligned}
E_{f_{n}}\left[L\left(d_{0}, \theta\right) \mid \boldsymbol{x}\right] & =E_{f_{n}}[L(\text { Aceitar } H, \theta) \mid \boldsymbol{x}] \\
& =\int_{\Theta}[b+c I(\theta \in T(\boldsymbol{x}))] f_{n}(\theta \mid \boldsymbol{x}) d \theta \\
& =\int_{\Theta} b f_{n}(\theta \mid \boldsymbol{x}) d \theta+\int_{T(x)} c f_{n}(\theta \mid \boldsymbol{x}) d \theta \\
& =b+c\left(1-\operatorname{ev}\left(\Theta_{H} \mid \boldsymbol{x}\right)\right) .
\end{aligned}
$$

Por outro lado, o risco a posteriori de não aceitação é

$$
\begin{aligned}
E_{f_{n}}\left[L\left(d_{1}, \theta\right) \mid \boldsymbol{x}\right] & =E_{f_{n}}[L(N \tilde{a} o \text { Aceitar } H, \theta) \mid \boldsymbol{x}] \\
& =\int_{\Theta} a[1-I(\theta \in T(\boldsymbol{x}))] f_{n}(\theta \mid \boldsymbol{x}) d \theta \\
& =\int_{\Theta} a f_{n}(\theta \mid \boldsymbol{x}) d \theta-\int_{T(x)} a f_{n}(\theta \mid \boldsymbol{x}) d \theta \\
& =a \operatorname{ev}\left(\Theta_{H} \mid \boldsymbol{x}\right) .
\end{aligned}
$$


O teste consiste em aceitar a hipótese $H$ se, e somente se, $E_{f_{n}}\left[L\left(d_{0}, \theta\right) \mid \boldsymbol{x}\right]<E_{f_{n}}\left[L\left(d_{1}, \theta\right) \mid \boldsymbol{x}\right]$, isto é, se

$$
e v\left(\Theta_{H} \mid \boldsymbol{x}\right)>\frac{b+c}{a+c}
$$

Considerando a desigualdade (3.4), observa-se que se $a<b$ então a decisão será sempre não aceitar a hipótese $H$, pois $e v\left(\Theta_{H} \mid \boldsymbol{x}\right)$ tem valores no intervalo [0,1]. Em particular, se tomar a decisão de não aceitar $H$ é sempre preferível do que aceitar $H$, mesmo com $\theta$ não pertencendo a $T(\boldsymbol{x})$, então a decisão será sempre não aceitar $H$.

Por outro lado, se $a \gg b$ e $c$ é relativamente pequeno, então para a aceitação de $H$ não é necessário um valor muito grande de $e v\left(\Theta_{H} \mid \boldsymbol{x}\right)$. Isso acontece quando a decisão é tomada considerando que $d_{0}$ é preferivel a $d_{1}$ e que $\theta$ pertencer a $T(x)$ não é um problema.

Exitem variações das funções de perda $L P_{1}$ cujas interpretações são diferentes da apresentada aqui que ainda satisfazem o FBST. Considere, por exemplo, a minimização da esperança a posteriori de $L^{\prime}$ definida por $L^{\prime}($ Não Aceitar $\mathrm{H}, \theta)=a-d I(\theta \in T(\boldsymbol{x}))$ e $L^{\prime}($ Aceitar $\mathrm{H}, \theta)=L($ Aceitar $\mathrm{H}, \theta)$ resultará novamente no FBST.

Uma abordagem diferente para o FBST é considerá-lo como um problema de estimação. Mais precisamente, considerar $e v\left(\Theta_{H} \mid \boldsymbol{x}\right)$ como um estimador de $I\left(\theta \in \Theta_{H}\right)$. Com isso, o espaço de decisão $D^{\prime}$ é formado pelas funções $\mathcal{A}$-mensuráveis $\phi: \mathcal{X} \rightarrow[0,1]$. Para mostrar que a medida de evidência do FBST é uma solução bayesiana desse problema define-se a seguinte função de perda

Definição 3.3.2. Seja $\phi(\boldsymbol{x})$ um estimador da função $I\left(\theta \in \Theta_{H}\right)$ e $T^{c}(\boldsymbol{x})$ o conjunto complementar de $T(\boldsymbol{x})$. A função de perda $L$ em $D^{\prime} \times \Theta$ definida por $L(\phi(\boldsymbol{x}), \theta)=$ $\left[I\left(\theta \in T^{c}(\boldsymbol{x})\right)-\phi(\boldsymbol{x})\right]^{2}$ é chamada função de perda $L P_{2}$.

Observe que a substituição de $I\left(\theta \in \Theta_{H}\right)$ por $I\left(\theta \in T^{c}(\boldsymbol{x})\right)=1-I(\theta \in T(\boldsymbol{x}))$ na expressão da função de perda $L P_{2}$ resultará na expressão da função de perda quadrática usual, cuja solução otimal é o verdadeiro estimador bayesiano $P\left(\theta \in \Theta_{H} \mid \boldsymbol{x}\right)$. O termo $I\left(\theta \in T^{c}(\boldsymbol{x})\right)$ incorpora a idéia original do FBST que pontos pertencentes a $T^{c}(\boldsymbol{x})$ devem garantir a hipótese precisa $H$ enquanto pontos pertencentes a $T(x)$ devem desacreditar a hipótese $H$. 
Teorema 3.3.2. A medida de evidência do FBST minimiza a esperança a posteriori da função de perda $\mathrm{LP}_{2}$.

Prova. O risco a posteriori é dado por

$$
\begin{aligned}
E_{f_{n}}[L(\phi(\boldsymbol{x}), \theta) \mid \boldsymbol{x}] & =\int_{T^{c}(x)} L(\phi(\boldsymbol{x}), \theta) f_{n}(\theta \mid \boldsymbol{x}) d \theta+\int_{T(x)} L(\phi(\boldsymbol{x}), \theta) f_{n}(\theta \mid \boldsymbol{x}) d \theta \\
& =\int_{T^{c}(x)}[1-\phi(\boldsymbol{x})]^{2} f_{n}(\theta \mid \boldsymbol{x}) d \theta+\int_{T(x)} \phi^{2}(\boldsymbol{x}) f_{n}(\theta \mid \boldsymbol{x}) d \theta \\
& =[1-\phi(\boldsymbol{x})]^{2} e v\left(\Theta_{H} \mid \boldsymbol{x}\right)+\phi^{2}(\boldsymbol{x})\left(1-e v\left(\Theta_{H} \mid \boldsymbol{x}\right)\right) \\
& =\phi^{2}(\boldsymbol{x})-2 \phi(\boldsymbol{x}) e v\left(\Theta_{H} \mid \boldsymbol{x}\right)+e v\left(\Theta_{H} \mid \boldsymbol{x}\right) \\
& =\left[\phi(\boldsymbol{x})-e v\left(\Theta_{H}, \boldsymbol{x}\right)\right]^{2}
\end{aligned}
$$

Portanto, $\phi^{*}(\boldsymbol{x})=e v\left(\Theta_{H} \mid \boldsymbol{x}\right)$ é a solução otimal que minimiza o risco a posteriori.

\subsection{Consistência do FBST}

Na presente Seção é brevemente discutido o conceito de estimador consistente e apresentada uma demonstração da consistência do FBST proposta por Borges e Stern [6].

Definição 3.4.1. Uma sequência de variáves aleatórias $Y_{n}$ definidas sobre um espaço amostral $\mathcal{Y}$ tende em probabilidade para uma constante $c\left(Y_{n} \stackrel{P}{\rightarrow}\right.$ c) se para todo a $>0$

$$
P\left[\left|Y_{n}-c\right|>a\right] \rightarrow 0 \text { quando } n \rightarrow \infty
$$

Uma sequência de estimadores $\sigma_{n}$ de $g(\theta)$ é consistente se para todo $\theta \in \Omega$

$$
\sigma_{n} \stackrel{P}{\rightarrow} g(\theta)
$$

Teorema 3.4.1. Considere a distribuição acumulada do e-valor contra uma hipótese $\Theta_{H}$, $\bar{V}(c)=\operatorname{Pr}[\overline{e v} \leq c]$, dado $\theta_{0}$, o valor verdadeiro do parâmetro. Sob condições apropriadas de regularidade, quando o tamanho amostral cresce $(n \rightarrow \infty)$ pode-se afirmar que

i) Se $\Theta_{H}$ é falsa $\left(\theta_{0} \notin \Theta_{H}\right)$ então $\overline{e v}$ converge em probabilidade para 1, isto é, $\bar{V}(0<c<1) \rightarrow 0$.

ii) Se $\Theta_{H}$ é verdadeira $\left(\theta_{0} \in \Theta_{H}\right)$ então $\bar{V}(c)$, o nível de confiança, é aproximado pela função

$$
Q Q(t, h, c)=Q\left(t-h, Q^{-1}(t, c)\right)
$$


onde $Q(k, x)=\frac{\Gamma(k / 2, x / 2)}{\Gamma(k / 2, \infty)}, \Gamma(k, x)=\int_{0}^{x} y^{k-1} e^{-y} d y, t=\operatorname{dim}(\Theta), h=\operatorname{dim}\left(\Theta_{H}\right)$ e $Q(k, x)$ é a distribuição acumulada da distribuição qui-quadrado com $k$ graus de liberdade.

Prova.

Sejam $\theta^{0}, \hat{\theta}$ e $\theta^{*}$ o valor verdadeiro, o máximo irrestrito da distribuição a posteriori e o máximo da distribuição a posteriori restrito a hipótese $\Theta_{H}$ do parâmetro $\theta$.

Como o FBST é invariante sob mudanças de variáveis, pode-se escolher um sistema de coordenadas tal que a matriz de informação de Fisher avaliada em $\theta^{0}$ é a identidade $\left(J\left(\theta^{0}\right)=I\right)$.

Pelo teorema de aproximação normal posteriori, para mais detalhes veja o apêndice $B$ de Gelman et al. [11], a diferença total padronizada entre $\hat{\theta}$ e $\theta^{0}$ converge em distribuição para a distribuição normal, isto é,

$$
\sqrt{n}\left(\hat{\theta}-\theta^{0}\right) \rightarrow N\left(0, J\left(\theta^{0}\right)^{-1} J\left(\theta^{0}\right) J\left(\theta^{0}\right)^{-1}\right)=N\left(0, J\left(\theta^{0}\right)^{-1}\right)=N(0, I) .
$$

Esta diferença total pode ser decomposta em duas componentes ortogonais: tangencial (à variedade definida pela hipótese) e transversal. Isto é, $d_{t}=d_{h}+d_{t-h}$ onde $d_{t}=\sqrt{n}\left(\hat{\theta}-\theta^{0}\right), d_{h}=\sqrt{n}\left(\theta^{*}-\theta^{0}\right)$ e $d_{t-h}=\sqrt{n}\left(\hat{\theta}-\theta^{*}\right)$.

Assim, considerando a norma $L_{2}$, as distâncias: total $\left\|d_{t}\right\|$, tangencial $\left\|d_{h}\right\|$ e transversal $\left\|d_{t-h}\right\|$ convergem em distribuição para variáveis qui-quadrado com $t, h$ e $t-h$ graus de liberdade, respectivamente.

Além disso, como o estimador de máximo a posteriori da matriz de informação de Fisher $\hat{J}$ converge em probabilidade para o valor verdadeiro $J\left(\theta^{0}\right)$ tem -se que o par $\left[\left\|d_{t-h}\right\|, \hat{J}\right]$ converge em distribuição para $\left[z, J\left(\theta^{0}\right)\right]$, onde z é uma variável qui-quadrado com $t-h$ graus de liberdade. Portanto, o valor de evidência contra $H, \overline{e v}(H)$, converge em distribuição para $\bar{e}=Q(t, z) \leq c \Longleftrightarrow z \leq Q^{-1}(t, c)$. Como z é uma variável qui-quadrada com $t-h$ graus de liberdade, tem-se

$$
\operatorname{Pr}(\bar{e} \leq c)=Q\left(t-h, Q^{-1}(t, c)\right)
$$




\subsection{Aspectos computacionais do FBST}

Nessa Seção são discutidos alguns detalhes úteis para a implementação numérica do cálculo do valor de evidência. Essa implementação é dividida em duas partes: otimização da surpresa relativa restrita ao conjunto definido pela hipótese precisa e integração da função de densidade a posteriori nos pontos onde a surpresa relativa é maior que o valor encontrado no processo de otimização.

Na parte de otimização será discutida a utilização do pacote de otimização ALGENCAN para encontrar o ponto $s_{n}^{*}=\sup _{\theta \in \Theta_{H}} s_{n}(\theta \mid \boldsymbol{x})$. Feito isso, será discutido o Método de Monte Carlo Importance Sampling para resolução do problema de integração.

\subsubsection{Otimização}

A procura pelo ponto de máxima surpresa relativa, restrito a hipótese precisa a ser testada, é fundamental para calcular o valor de evidência contra essa hipótese.

A falta de rotinas de otimização nos softwares estatísticos capazes de tratar problemas com restrições não lineares de igualdade e desigualdade motivou a busca por uma rotina de otimização que tratasse esse tipo de problema de maneira satisfatória e fosse compatível com outras linguagens de programação.

No presente trabalho, a opção escolhida para superar essa dificuldade foi a utilização do pacote ALGENCAN que foi desenvolvida originalmente em linguagem Fortran pelo grupo do projeto TANGO (Trustable Algorithms for Nonlinear General Optimization). Esse pacote foi escolhido por ser um software livre, ter suporte online, ser constituído de códigos fáceis de usar que requerem somente conhecimentos básicos de otimização para sua utilização e possuir interface para várias linguagens de programação, como: R, C, Octave, Matlab, Java, entre outras. Em todas essas interfaces, o usuário precisa somente editar um pequeno script que contem as definições do problema (função objetivo, restrições, gradientes e hessianas) e alguns parâmetros opcionais como as ordens de grandezas admitidas para a convergência do algoritmo.

O ALGENCAN foi desenvolvido para resolver eficientemente problemas de otimização 
definidos da seguinte forma

$$
\begin{array}{lll}
\text { Minimizar } & f(x) & \\
\text { Sujeito a } & c_{j}(x)=0 \quad j \in E \\
& c_{j}(x) \leq 0 \quad j \in I \\
& l \leq x \leq u &
\end{array}
$$

onde $x \in \mathrm{R}^{n}$ é um vetor de incógnitas, $f: \mathrm{R}^{n} \rightarrow \mathrm{R}$ é a função objetivo do problema, $c: \mathrm{R}^{n} \rightarrow \mathrm{R}^{m}$ é a função de restrições, $E$ é o conjunto de índices das restrições de igualdade e $I$ é o conjunto de índices das restrições de desigualdade.

O ALGENCAN é uma implementação do método do lagrangeano aumentado que requer a avaliação da função de restrições $c$, da função objetivo $f$, do vetor gradiente de $f$ e da matriz hessiana de $f$. Para o vetor gradiente e a matriz hessiana de $f$, a rotina possui um método de aproximação que é utilizado quando essas informações não são fornecidas pelo usuário. Entretanto, para obter melhor desempenho computacional desse pacote recomenda-se fortemente ao usuário que forneça essas informações ao programa.

Uma descrição introdutória do método do lagrangeano aumentado pode ser encontrada nos livros de Luenberger e Ye [18], Nocedal e Wright [22] e Fletcher [10], sendo os dois últimos com maior ênfase no aspecto computacional. Para encontrar informações mais detalhadas sobre as funções de penalidade, critérios de convergência e experimentos numéricos utilizando o método do lagrangeano aumento consulte o trabalho de Birgin, Castillo e Martínez [5]. Maiores detalhes sobre o pacote ALGENCAN podem ser encontradas nos artigos de Andreani et al. [1] e [2].

\subsubsection{Integração}

Depois de encontrar o ponto onde a função de surpresa a posteriori atinge seu valor máximo, chega-se a segunda parte do cálculo do valor de evidência, que é avaliar a integral da função de densidade a posteriori no conjunto de curva de nível definido por esse ponto de máximo.

Como esse conjunto geralmente não define uma região de simples integração, uma maneira eficiente de realizar esse processo de integração é por meio de uma aproximação numérica utilizando o método de Monte Carlo que fornece aproximações satisfatórias 
mesmo para distribuições a posteriori definidas em espaços paramétricos de grandes dimensões. Maiores detalhes sobre a utlização do método de Monte Carlo em problemas de integração podem ser encontrados em Evans e Swartz [9], Stern [38] e Zacks e Stern [42].

Para calcular o valor de evidência contra uma hipótese $H$ é preciso estimar a razão

$$
\overline{e v}\left(\Theta_{H} \mid \boldsymbol{x}\right)=\frac{\int_{T} s_{n}(\theta) d \theta}{\int_{\Theta} s_{n}(\theta) d \theta},
$$

onde $T=\left\{\theta \in \Theta \mid s_{n}(\theta)>s^{*}\right\}$.

Como o espaço $\Theta$ pode ser ilimitado, é conveniente escolher aleatoriamente os valores de $\theta$ com uma distribuição $g(\theta)$, positiva em $\Theta$. Assim, a função de evidência definida em (3.7) é equivalente a

$$
\overline{e v}\left(\Theta_{H} \mid \boldsymbol{x}\right)=\frac{\int_{\Theta} Z_{g}^{*}(\theta \mid \boldsymbol{x}) g(\theta) d \theta}{\int_{\Theta} Z_{g}(\theta \mid \boldsymbol{x}) g(\theta) d \theta},
$$

onde $Z_{g}(\theta \mid \boldsymbol{x})=\frac{s_{n}(\theta \mid \boldsymbol{x})}{g(\theta)}$ e $Z_{g}^{*}(\theta \mid \boldsymbol{x})=I(\theta \in T) Z_{g}(\theta \mid \boldsymbol{x})$.

Assim, a estimativa pelo método de Monte Carlo para o valor de evidência é

$$
\hat{E} v_{g, m}(\boldsymbol{x})=\frac{\sum_{i=1}^{m} Z_{g}^{*}\left(\theta^{i} \mid \boldsymbol{x}\right)}{\sum_{i=1}^{m} Z_{g}\left(\theta^{i} \mid \boldsymbol{x}\right)}
$$

onde os $\theta^{i}, i=1, \ldots, m$ são idd e sorteados independentemente em $\Theta$ com distribuição $g(\theta)$. Disso tem-se que

$$
\hat{E} v_{g, m}(\boldsymbol{x}) \rightarrow e v\left(\Theta_{H} \mid \boldsymbol{x}\right) \text { quando } m \rightarrow \infty .
$$

Para determinar o valor de $m$, número mínimo de amostras geradas computacionalmente com distribuição $g(\theta)$ em cada simulação numérica suficiente para garantir uma boa precisão da estimativa da evidência, é necessário estimar a precisão do método de Monte Carlo. Assim, com $m$ suficientemente grande fixado, a distribuição assintótica de $\hat{E} v_{g, m}(\boldsymbol{x})$ é normal com média $e v\left(\Theta_{H} \mid \boldsymbol{x}\right)$ e variância assintótica $V_{g}(\boldsymbol{x})$ que, pelo método Delta (veja Lehmann e Casella [16]), é dada por

$$
V_{g}(\boldsymbol{x})=\frac{1}{m}\left(\frac{\sigma_{g}^{* 2}}{\mu_{g}{ }^{2}}+\frac{{\sigma_{g}}^{2} \mu_{g}^{* 2}}{\mu_{g}{ }^{4}}-2 \frac{\mu_{g}^{*}}{\mu_{g}{ }^{3}} \gamma_{g}\right),
$$


onde

$$
\begin{aligned}
\mu_{g} & =\int_{\Theta} Z_{g}(\theta \mid \boldsymbol{x}) g(\theta) d \theta \\
\mu_{g}^{*} & =\int_{\Theta} Z_{g}^{*}(\theta \mid \boldsymbol{x}) g(\theta) d \theta \\
\sigma_{g}^{2} & =\int_{\Theta}\left[Z_{g}(\theta \mid \boldsymbol{x})-\mu_{g}\right]^{2} g(\theta) d \theta \\
\sigma_{g}^{* 2} & =\int_{\Theta}\left[Z_{g}^{*}(\theta \mid \boldsymbol{x})-\mu_{g}^{*}\right]^{2} g(\theta) d \theta \\
\gamma_{g}^{2} & =\int_{\Theta}\left[Z_{g}(\theta \mid \boldsymbol{x})-\mu_{g}\right]\left[Z_{g}^{*}(\theta \mid \boldsymbol{x})-\mu_{g}^{*}\right] g(\theta) d \theta
\end{aligned}
$$

são os valores esperados, variâncias e covariâncias de $Z(\theta \mid \boldsymbol{x})$ e $Z^{*}(\theta \mid \boldsymbol{x})$ com respeito a $g(\theta)$

Definindo os coeficientes

$$
\xi_{g}=\frac{\sigma_{g}}{\mu_{g}} \quad \text { e } \quad \xi_{g}^{*}=\frac{\sigma_{g}^{*}}{\mu_{g}^{*}},
$$

e denotado $\eta=\mu_{g}^{*} / \mu_{g}$, pode-se escrever a variância assintótica como

$$
V_{g}(\boldsymbol{x})=\frac{1}{m}\left(\xi_{g}^{* 2}+\eta^{2} \xi_{g}^{2}-2 \frac{\eta \gamma_{g}}{\mu_{g}^{2}}\right) .
$$

Definindo as variáveis complementares

$$
\begin{aligned}
Z_{g}^{c}(\theta \mid \boldsymbol{x}) & =I^{c}(\theta \mid \boldsymbol{x}) Z_{g}(\theta \mid \boldsymbol{x}) \\
I^{c}(\theta \mid \boldsymbol{x}) & =1-I^{*}(\theta \mid \boldsymbol{x}) \\
\sigma_{g}^{c 2} & =V_{g}\left(Z^{c}(\theta \mid \boldsymbol{x})\right) \\
\xi_{g}^{c} & =\frac{\sigma_{g}^{c}}{\mu_{g}}
\end{aligned}
$$

e realizando algumas manipulações algébricas é possível escrever $V_{g}(\boldsymbol{x})$ em função de $\xi_{g}^{*}$ e $\xi_{g}^{c}$

$$
V_{g}(\boldsymbol{x})=\frac{1}{m}\left[\xi_{g}^{* 2}(1-\eta)^{2}+\xi_{g}^{c 2} \eta^{2}+2 \eta^{2}(1-\eta)^{2}\right]
$$

Para valores grandes de $m$, o intervalo de confiança assintótico $(1-\beta)$ para $\eta$ é $\hat{E} v_{g, m}(\boldsymbol{x}) \pm \Delta_{g, m, \beta}$, onde

$$
\Delta_{g, m, \beta}^{2}=\frac{F_{1-\beta}(1, m)}{m}\left[\hat{\xi}_{g}^{*}(1-\hat{\eta})^{2}+\hat{\xi}_{g}^{c} \hat{\eta}^{2}+2 \hat{\eta}^{2}(1-\hat{\eta})^{2}\right]
$$


onde $F_{1-\beta}(1, m)$ é o quantile $(1-\beta)$ da distribuição $F(1, m)$, e $\hat{\eta}, \hat{\xi}_{g}^{*}$ e $\hat{\xi}_{g}^{c}$ são estimadores consistentes das respectivas quantidades.

Portanto, para $m$ suficientemente grande, pode-se utilizar a aproximação

$$
\Delta_{g, m, \beta}^{2}=\frac{\chi_{1-\beta}^{2}(1)}{m}\left[\hat{\xi}_{g}^{2}(1-\hat{\eta})^{2}+\hat{\xi}_{g}^{2} \hat{\eta}^{2}+2 \hat{\eta}^{2}(1-\hat{\eta})^{2}\right]
$$

pois $F(1, m)$ converge em distribuição para a distribuição qui-quadrada com 1 grau de liberdade quando $m \rightarrow \infty$.

$$
m \geq \frac{\chi_{1-\beta}^{2}(m)}{\sigma^{2}}\left[\hat{\xi}_{g}^{2}(1-\hat{\eta})^{2}+\hat{\xi}_{g}^{2} \hat{\eta}^{2}+2 \hat{\eta}^{2}(1-\hat{\eta})^{2}\right]
$$




\section{Simulações numéricas}

Neste capítulo são descritas as simulações computacionais realizadas no presente trabalho juntamente com os resultados numéricos obtidos. Estas simulações têm por objetivo avaliar o comportamento da distribuição amostral quando a distribuição das probabilidades de seleção da amostra é desconhecida por quem faz a análise dos dados e o valor esperado dessas probabilidade de seleção é modelado por um polinômio aproximador de baixa ordem dependendo da variável resposta. Para determinar o polinômio de menor grau que ajusta os dados com uma precisão satisfatória foi utilizado o valor de evidência do FBST.

Esse simulações foram realizadas baseadas no trabalho de Pfeffermann, Moura e Silva [31] considerando como variável resposta do modelo a proeficiência de alunos e variáveis concomitantes dummies representando as seguintes características: gênero (1 para alunos do sexo masculino), idade (1 para alunos entre 15 e 16 anos), idade (1 para alunos com 17 anos ou mais) e escolaridade dos pais (1 caso pelo menos um dos dois tenha curso universitário completo).

Assim, considere que a distribuição populacional para a variável resposta $Y$ é especificada corretamente com distribuição normal e variáveis concomitantes representadas pela matriz $X=\left[\boldsymbol{x}_{1}, \ldots, \boldsymbol{x}_{N}\right]^{T}$ contendo em cada linha $i$ as informações do individuo $i$, tal que

$$
y_{i} \mid \boldsymbol{X}_{i}=\boldsymbol{x}_{i}, \boldsymbol{\beta}, \sigma^{2} \sim N\left(\boldsymbol{x}_{i}^{T} \boldsymbol{\beta}, \sigma^{2}\right), i=1, \ldots, N,
$$

onde $\boldsymbol{\beta}=\left(\beta_{1}, \ldots, \beta_{p}\right) \in \mathrm{R}^{p}$ é o conjunto de parâmetros a serem estimados no modelo, $X$ é uma matriz de dados $N \times p$ cujas entradas da primeira coluna são todas iguais a 1 e a variância $\sigma^{2}=693$ é conhecida. Os valores de $\boldsymbol{\beta}$ (listados nas tabelas 4.1-4.11) 
e $\sigma^{2}$ utilizados no presente estudo também foram escolhidos baseados nas simulações computacionais realizadas por Pfeffermann, Moura e Silva [31].

Além disso, vamos supor que $n$ unidades da população são selecionadas com probabilidade proporcional ao seu tamanho, com os tamanhos $z_{i}$ satisfazendo a distribuição log-normal tal que

$$
\log \left(z_{i}\right) \mid Y_{i}=y_{i}, \gamma_{1}, \gamma_{2}, \sigma_{z}^{2} \sim N\left(\gamma_{1}+\gamma_{2} y_{i}, \sigma_{z}^{2}\right), i=1, \ldots, N
$$

onde $\gamma_{1}$ e $\gamma_{2}$ são parâmetros desconhecidos a serem estimados e a variância $\sigma_{z}^{2}=1$ é conhecida. Os valores de $\gamma_{1}=2,5$ e $\gamma_{2}=0,0075$ também foram escolhidos baseados na simulações computacionais realizadas por Pfeffermann, Moura e Silva [31].

Assim, sob a condição (4.2) a amostragem é informativa quando $\gamma_{2} \neq 0$, pois os tamanhos $z_{i}$ utilizados no processo de seleção da amostra dependem dos valores da variável resposta $y_{i}$.

No processo de inferência dos dados, a modelagem da probabilidade de seleção da amostra será realizada considerando dois casos: o primeiro deles, quando essa distribuição é conhecida; e o segundo, quando essa informação não está disponível para quem faz a análise dos dados e o valor esperado das probabilidades de seleção da amostra é modelado por um polinômio aproximador de baixa ordem dependendo da variável resposta $y_{i}$ via séries de Taylor. Observe que o segundo caso deve ser considerado também quando o analista que faz a análise dos dados não sabe se a amostragem realizada é informativa. Esses dois casos são descritos na seções seguintes.

\subsection{Distribuição das probabilidades de seleção da amos- tra conhecida}

Considere o modelo populacional definido por (4.1) e suponha que as amostras são selecionadas com probabilidade proporcional ao tamanho, com os tamanhos satisfazendo (4.2). Assim, quando essa distribuição é conhecida durante a análise dos dados, tem-se

$$
\pi_{i}=\operatorname{Pr}\left(I_{i}=1 \mid Z_{U}\right)=n \frac{z_{i}}{\sum_{i=1}^{N} z_{i}}=\frac{n}{N \bar{z}} z_{i}=C z_{i}
$$

onde $\bar{z}=\frac{1}{N} \sum_{i=1}^{N} z_{i}$ é a média populacional dos tamanhos $z$ e $C=\frac{n}{N \bar{z}}$ é uma constante. 
Utilizando propriedades da distribuição log-normal, tem-se

$$
\begin{aligned}
E_{p}\left(\pi_{i} \mid \boldsymbol{x}_{i}, y_{i}, \gamma_{1}, \gamma_{2}, \sigma_{z}^{2}\right) & =C E_{p}\left(z_{i} \mid \gamma_{1}, \gamma_{2}, \sigma_{z}^{2}, y_{i}, \boldsymbol{x}_{i}\right) \\
& =C \exp \left[\gamma_{1}+\gamma_{2} y_{i}+\frac{\sigma_{z}^{2}}{2}\right]
\end{aligned}
$$

Integrando a expressão (4.4) em relação a $y_{i}$, obtem-se

$$
E_{p}\left(\pi_{i} \mid \boldsymbol{x}_{i}, \gamma_{1}, \gamma_{2}, \sigma_{z}^{2}\right)=C \exp \left[\gamma_{1}+\gamma_{2} \boldsymbol{x}_{i}^{T} \boldsymbol{\beta}+\frac{\gamma_{2}^{2} \sigma^{2}+\sigma_{z}^{2}}{2}\right] .
$$

O modelo populacional (4.1) com amostragem proporcional ao tamanho satisfazendo (4.2) é um dos casos de invariância sob amostragem que se encaixa nas hipóteses da Proposição 2.5.1. Em virtude disso, a distribuição amostral é dada por

$$
f_{s}\left(y_{i} \mid \boldsymbol{x}_{i}, \boldsymbol{\beta}, \sigma^{2}, \gamma_{2}\right)=N\left(\boldsymbol{x}_{i}^{T} \boldsymbol{\beta}+\gamma_{2} \sigma^{2}, \sigma^{2}\right)
$$

Assim, quando $\gamma_{2} \neq 0$, a distribuição amostral de $z_{i} \mid \gamma_{1}, \gamma_{2}, \sigma_{z}^{2}, y_{i}$ deve ser considerada no processo de inferência dos parâmetros $\boldsymbol{\beta}$ e $\sigma^{2}$. De tal forma que é necessário construir o modelo amostral $f_{s}\left(\log \left(z_{i}\right) \mid y_{i}, \gamma_{1}, \gamma_{2}, \sigma_{z}^{2}\right)$ e incluí-lo na distribuição conjunta a posteriori dos parâmetros. Dessa maneira, tem-se

$$
E_{p}\left(\pi_{i} \mid y_{i}, z_{i}, \gamma_{1}, \gamma_{2}, \sigma_{z}^{2}\right)=C z_{i}
$$

E por consequência,

$$
E_{p}\left(\pi_{i} \mid y_{i}, \gamma_{1}, \gamma_{2}, \sigma_{z}^{2}\right)=C E_{p}\left(z_{i} \mid y_{i}, \gamma_{1}, \gamma_{2}, \sigma_{z}^{2}\right)=C \exp \left[\gamma_{1}+\gamma_{2} y_{i}+\frac{\sigma_{z}^{2}}{2}\right]
$$

Por conseguinte, utilizando novamente a Proposicão 2.5.1, a distribuição amostral de $\log \left(z_{i}\right) \mid y_{i}, \gamma_{1}, \gamma_{2}, \sigma_{z}^{2}$ é dada por

$$
f_{s}\left(\log \left(z_{i}\right) \mid y_{i}, \gamma_{1}, \gamma_{2}, \sigma_{z}^{2}\right)=N\left(\gamma_{1}+\gamma_{2} y_{i}+\sigma_{z}^{2}, \sigma_{z}^{2}\right)
$$

Observe que neste caso a diferença entre a distribuição populacional (4.2) e a distribuição amostral (4.9) é somente a adição do termo $\sigma_{z}^{2}$ no intercepto do modelo.

Considerando a distribuição a priori não informativa $f\left(\boldsymbol{\beta}, \gamma_{1}, \gamma_{2}\right)=1$, a distribuição conjunta a posteriori pode ser escrita como 
$f\left(\boldsymbol{\beta}, \gamma_{1}, \gamma_{2} \mid X, \boldsymbol{y}, \log (\boldsymbol{z}), \sigma^{2}, \sigma_{z}^{2}\right) \propto$

$$
\begin{aligned}
& \propto \prod_{i=1}^{n}\left[f_{s}\left(\log \left(z_{i}\right) \mid y_{i}, \gamma_{1}, \gamma_{2}, \sigma_{z}^{2}\right) f_{s}\left(y_{i} \mid \boldsymbol{x}_{i}, \boldsymbol{\beta}, \sigma^{2}, \gamma_{2}\right)\right] \\
& \propto \prod_{i=1}^{n}\left[N\left(\gamma_{1}+\gamma_{2} y_{i}+\sigma_{z}^{2} \mid \log \left(z_{i}\right), \sigma_{z}^{2}\right) N\left(x_{i}^{T} \boldsymbol{\beta}+\gamma_{2} \sigma^{2} \mid y_{i}, \sigma^{2}\right)\right] .
\end{aligned}
$$

Assim, o logaritmo da distribuição conjunta a posteriori é dado por

$$
\begin{aligned}
& l f\left(\boldsymbol{\beta}, \gamma_{1}, \gamma_{2} \mid X, \boldsymbol{y}, \log (\boldsymbol{z}), \sigma^{2}, \sigma_{z}^{2}\right) \propto \\
& \quad \propto \sum_{i=1}^{n} \log \left[N\left(\gamma_{1}+\gamma_{2} y_{i}+\sigma_{z}^{2} \mid \log \left(z_{i}\right), \sigma_{z}^{2}\right)\right]+\sum_{i=1}^{n} \log \left[N\left(\boldsymbol{x}_{i}^{T} \boldsymbol{\beta}+\gamma_{2} \sigma^{2} \mid y_{i}, \sigma^{2}\right)\right] .
\end{aligned}
$$

Portanto, as derivadas parciais de (4.11) são dadas por

$$
\begin{aligned}
\frac{\partial}{\partial \beta_{k}} l f\left(\boldsymbol{\beta}, \gamma_{1}, \gamma_{2} \mid X, \boldsymbol{y}, \log (\boldsymbol{z}), \sigma^{2}, \sigma_{z}^{2}\right) & \propto \frac{1}{\sigma^{2}} \sum_{i=1}^{n}\left(y_{i}-\boldsymbol{x}_{i}^{T} \boldsymbol{\beta}-\gamma_{2} \sigma^{2}\right) x_{i k} \\
\frac{\partial}{\partial \gamma_{1}} l f\left(\boldsymbol{\beta}, \gamma_{1}, \gamma_{2} \mid X, \boldsymbol{y}, \log (\boldsymbol{z}), \sigma^{2}, \sigma_{z}^{2}\right) & \propto \frac{1}{\sigma_{z}^{2}} \sum_{i=1}^{n}\left(\log \left(z_{i}\right)-\gamma_{1}-\gamma_{2} y_{i}-\sigma_{z}^{2}\right) \\
\frac{\partial}{\partial \gamma_{2}} l f\left(\boldsymbol{\beta}, \gamma_{1}, \gamma_{2} \mid X, \boldsymbol{y}, \log (\boldsymbol{z}), \sigma^{2}, \sigma_{z}^{2}\right) & \propto \frac{1}{\sigma_{z}^{2}} \sum_{i=1}^{n}\left[\left(\log \left(z_{i}\right)-\gamma_{1}-\gamma_{2} y_{i}-\sigma_{z}^{2}\right) y_{i}\right] \\
& +\sum_{i=1}^{n}\left(y_{i}-\boldsymbol{x}_{i}^{T} \boldsymbol{\beta}-\gamma_{2} \sigma^{2}\right)
\end{aligned}
$$

\subsection{Distribuição das probabilidades de seleção da amos- tra desconhecida}

Quando a distribuição das probabilidades de seleção é desconhecida, sob algumas condições de estabilidade o valor esperado das probabilidades de seleção da amostra pode ser modelado via séries de Taylor por um polinômio aproximador dependendo da variável resposta $Y_{i}$ da forma

$$
E_{p}\left(z_{i} \mid y_{i}, \eta_{1}, \cdots, \eta_{d}\right)=\eta_{0}+\eta_{1} y_{i}+\eta_{2} y_{i}^{2}+\cdots+\eta_{d} y_{i}^{d} \text {, para algum } d \geq 0
$$




\subsection{Distribuição das probabilidades de seleção da amostra desconhecida 53}

Utilizando a expressão (2.31) e considerando a distribuição a priori não-informativa $f\left(\boldsymbol{\beta}, \eta_{0}, \ldots, \eta_{d}\right)=1$, a distribuição conjunta a posteriori pode ser escrita como

$$
f\left(\boldsymbol{\beta}, \eta_{0}, \ldots, \eta_{d} \mid X, \boldsymbol{y}, \log (\boldsymbol{z}), \sigma^{2}, \sigma_{z}^{2}\right) \propto \prod_{i=1}^{n} \frac{z_{i} N\left(\boldsymbol{x}_{i}^{T} \boldsymbol{\beta} \mid y_{i}, \sigma^{2}\right) N\left(\sum_{j=0}^{d} \eta_{j} y_{i}^{j} \mid z_{i}, \sigma_{z}^{2}\right)}{\eta_{0}+\sum_{j=1}^{d} \eta_{j} E_{p}\left(y_{i}^{j} \mid \boldsymbol{\beta}, \sigma^{2}, \boldsymbol{x}_{i}\right)} .
$$

Assim, o logaritmo da distribuição conjunta a posteriori pode ser escrita como

$$
\begin{aligned}
l f\left(\boldsymbol{\beta}, \eta_{0}, \ldots, \eta_{d} \mid X, \boldsymbol{y}, \log (\boldsymbol{z}), \sigma^{2}, \sigma_{z}^{2}\right) & \propto \sum_{i=1}^{n} \log \left(z_{i}\right)+\sum_{i=1}^{n} \log \left[N\left(\sum_{j=0}^{d} \eta_{j} y_{i}^{j} \mid z_{i}, \sigma_{z}^{2}\right)\right] \\
& +\sum_{i=1}^{n} \log \left[N\left(\boldsymbol{x}_{i}^{T} \boldsymbol{\beta} \mid y_{i}, \sigma^{2}\right)\right] \\
& -\sum_{i=1}^{n} \log \left[\sum_{j=0}^{d} \eta_{j} E_{p}\left(y_{i}^{j} \mid \boldsymbol{\beta}, \sigma^{2}, \boldsymbol{x}_{i}\right)\right] .
\end{aligned}
$$

Consequentemente, as derivadas parciais são

$$
\begin{aligned}
\frac{\partial}{\partial \beta_{k}} l f\left(\boldsymbol{\beta}, \eta_{0}, \ldots,\right. & \left.\eta_{d} \mid X, \boldsymbol{y}, \log (\boldsymbol{z}), \sigma^{2}, \sigma_{z}^{2}\right) \propto \\
& \propto \frac{1}{\sigma^{2}}(\boldsymbol{y}-X \boldsymbol{\beta})^{T} \boldsymbol{x}_{\cdot k}-\sum_{i=1}^{n} \frac{\left[\sum_{j=0}^{d} \eta_{j} \frac{\partial}{\partial \beta_{k}} E_{p}\left(y_{i}^{j} \mid \boldsymbol{\beta}, \sigma^{2}, \boldsymbol{x}_{i}\right)\right]}{\left[\sum_{j=0}^{d} \eta_{j} E_{p}\left(y_{i}^{j} \mid \boldsymbol{\beta}, \sigma^{2}, \boldsymbol{x}_{i}\right)\right]},
\end{aligned}
$$

e

$$
\begin{aligned}
\frac{\partial}{\partial \eta_{k}} l f\left(\boldsymbol{\beta}, \eta_{0}, \ldots, \eta_{d} \mid X, \boldsymbol{y}, \log (\boldsymbol{z}), \sigma^{2}, \sigma_{z}^{2}\right) & \propto \\
& \propto \frac{1}{\sigma_{z}^{2}} \sum_{i=1}^{n} y_{i}^{k}\left(z_{i}-\sum_{j=0}^{d} \eta_{j} y_{i}^{j}\right)-\sum_{i=1}^{n} \frac{E_{p}\left(y_{i}^{k} \mid \boldsymbol{\beta}, \sigma^{2}, \boldsymbol{x}_{i}\right)}{\left[\sum_{j=0}^{d} \eta_{j} E_{p}\left(y_{i}^{j} \mid \boldsymbol{\beta}, \sigma^{2}, \boldsymbol{x}_{i}\right)\right]}
\end{aligned}
$$

Considerando que o modelo populacional é especificado corretamente, para decidir se o processo de amostragem é informativo, é necessário avaliar qual é o grau $d$ do polinômio aproximador dependendo da variável resposta $\boldsymbol{Y}$ na distribuição conjunta a posteriori dada por (4.15) que melhor ajusta os dados. Se $\eta_{j} \neq 0$ para algum $j>1$, então o processo de amostragem é informativo e a distribuição conjunta a posteriori deve ser utilizada na estimação dos parâmetros.

Para decidir isso, a idéia é começar testando se $\eta_{1}=0$ considerando $\eta_{j}=0, \forall j>1$. Isso pode ser feito utilizando o FBST para testar se a hipótese precisa $H_{1}: \eta_{1}=0$ é 
aceita ou não. Quando este teste de hipótese é realizado, o que está sendo testado é se distribuição populacional ajusta melhor os dados do que a distribuição amostral com $\eta_{1} \neq 0$ e $\eta_{j}=0, \forall j>1$.

Mesmo quando a hipótese $H_{1}$ é aceita, é aconselhável testar pelo menos se $\eta_{2}=0$ considerando $\eta_{j}=0, \forall j>2$ antes de concluir que o processo de amostragem é ignorável e que as probabilidades de seleção da amostra não fornecem novas informações além daquelas já fornecidas pelo processo de amostragem.

Assim, quando uma hipótese $H_{j-1}$ não é aceita para algum valor de $j>1$, é recomendável continuar testando as seguintes hipóteses

$$
H_{k}:\left(\eta_{0}, \eta_{1}, \ldots, \eta_{k}\right) \in\left\{\left(\eta_{0}, \eta_{1}, \ldots, \eta_{k}\right) \mid \eta_{k}=0\right\}, \text { for } k=j, j+1, \ldots
$$

até encontrar um valor $d$ tal que a hipótese $H_{d}$ não é aceita e $H_{d+1}$ é aceita, isto é suficiente para garantir que $\eta_{d} \neq 0$ e $\eta_{d+1}=0$.

Uma hipótese $H_{j}$ deve ser aceita quando o valor de evidência contra $H_{j}$ é maior do que o nível de confiança dado por

$$
Q Q(t, h, c)=Q\left(t-h, Q^{-1}(t, c)\right)
$$

onde

$$
Q(k, x)=\frac{\Gamma(k / 2, x / 2)}{\Gamma(k / 2, \infty)} \text { and } \Gamma(k, x)=\int_{0}^{x} y^{k-1} e^{-y} d y,
$$

$t$ é a dimensão do espaço de parâmetros, $h$ é a dimensão da hipótese e $Q(k, x)$ é a distribuição qui-quadrada acumulada com $k$ graus de liberdade.

\subsection{Seleção da amostra}

Considerando as medidas $z_{i}$ conhecidas no processo de amostragem, os métodos para selecionar as amostras com probabilidade proporcional ao tamanho utilizados nas simulações computacionais do presente Capítulo foram:

i) Amostragem com probabilidade proporcional ao tamanho com reposição: Sorteia-se uma unidade de cada vez com reposição com probabilidade proporcional a $z_{j}$ até que $n$ unidades distintas tenham sido selecionadas. 
ii) Amostragem pelo método de Sampford: Sorteia-se a primeira unidade com probabilidade $p_{j}=z_{j} / N \bar{z}$ e as demais $n-1$ unidades com probabilidade proporcional a $p_{j} /\left(1-n p_{j}\right)$ com reposição. Se uma unidade for sorteada mais de uma vez, a amostra é rejeitada e o processo de amostragem é iniciado novamente.

iii) Amostragem rejeitiva: Sorteia-se uma unidade de cada vez com reposição, com probabilidade proporcional a $\alpha_{j}=p_{j} /\left(1-p_{j}\right)$, rejeita-se da amostra as unidades já selecionadas se ocorrer repetição e, nesse caso, inicia-se o processo de amostragem inteiro novamente. Este método de amostragem é equivalente a usar amostragem condicional de Poisson para $n$ unidades distintas.

iv) Amostragem sucessiva: Sorteia-se uma unidade de cada vez com reposição, com probabilidades $p_{j}$ até que $n$ unidades distintas tenham sido selecionadas. Este método de amostragem é equivalente ao sorteio das unidades em sequência sem reposição tal que no $(r+1)$-ésimo sorteio a probabilidade de seleção da unidade $j$, não selecionada anteriormente, é $P(j \in S)=z_{j} / \sum_{k \notin S_{r}}^{N} z_{k}$ onde $S_{r}$ denota as unidades selecionadas nos $r$ primeiros sorteios.

v) Amostragem sistemática: Sorteia-se um número aleatório $r$ entre 0 e $\bar{z}=\frac{1}{n} \sum_{i=1}^{N} z_{i}$ e então para cada $u=r+(i-1) \bar{z}$, com $i=1, \ldots, n$, é selecionada para a amostra a primeira unidade $k$ da população tal que $\sum_{i=1}^{k} z_{i}<u$. Mais detalhes sobre este método de amostragem podem ser encontrados no trabalho de Bellhouse [4].

\subsection{Implementação das simulações}

A simulação computacional consiste dos vários passos descritos abaixo. Nos passos i, ii e iii os dados populacionais (variáveis concomitantes, váriavel resposta e variáveis do processo amostral) foram gerados de acordo com a distribuição populacional e uma amostra de cada populacão foi sorteada. Nos passos $i v$ e $v$, foi realizada a estimação dos parâmetros utilizando: a distribuição populacional, a distribuição amostral considerando a distribuição das probabilidades de seleção da amostra conhecida e a distribuição amostral aproximando essa distribuição por polinômios. Os passos $i-v$ foram repetidos 500 vezes.

i) Gerar $N=500$ medidas populacionais independentes $\left\{Y_{1}, Y_{2}, \ldots, Y_{N}\right\}$ da distibuição 
populacional de $y_{i} \mid \boldsymbol{\beta}, \sigma^{2}, \boldsymbol{X}_{i}=\boldsymbol{x}_{i}$ dada em (4.1) e com os valores de $\boldsymbol{x}_{i}$ gerados com distribuição $\operatorname{Gama}\left(\alpha_{x}, \beta_{x}\right)$, com $\alpha_{x}$ e $\beta_{x}$ escolhidos com distribuição uniforme no intervalo $[0,1]$.

ii) Gerar medidas populacionais para as variáveis de desenho $Z_{i}$ satisfazendo a distribuição descrita em (4.2), com $\gamma_{0}=2,5, \gamma_{1}=0,0075$ e $\sigma_{z}=1$.

iii) Para cada população, selecionar com probabilidade proporcional ao tamanho uma amostra de tamanho $n=50$ empregando algum dos seguintes métodos de seleção da amostra:

iii $i_{1}$ ) Amostragem aleatória simples.

$\left.i i i_{2}\right)$ Amostragem proporcional ao tamanho com reposição.

iii $i_{3}$ ) Amostragem sucessiva.

iii 4 ) Amostragem pelo método de Sampford.

$\left.i_{i i}\right)$ Amostragem rejeitiva.

$\left.i i i_{6}\right)$ Amostragem sistemática.

iv) Estimar os parâmetros de interesse considerando que a distribuição das probabilidades de seleção da amostra é desconhecida, isto é, utilizando a distribuição conjunta a posteriori definida em (4.15) com o grau do polinômio aproximador crescendo até encontrar um valor $d$ tal que o valor de evidência seja maior que $Q Q(t, h, 0.95)$, onde $Q Q$ é a função definida em (4.18), $t$ é a dimensão do espaço de parâmetros e $h$ é a dimensão da hipótese.

v) Estimar os parâmetros de interesse considerando que a distribuição das probabilidades de seleção da amostra é conhecida, isto é, utilizando a distribuição conjunta a posteriori definida em (4.10).

Observe que o método de amostragem $i i_{1}$ não é informativo e foi utilizado como referência para avaliar os efeitos da amostragem na estimação dos parâmetros. Por outro lado, os métodos de amostragem $i i i_{2}-i i i_{6}$ são informativos e satisfazem as hipóteses da Proposição 2.5.1 do Capítulo 2 do presente trabalho.

Além disso, os parâmetros estimados considerando a distribuição das probabilidades de seleção da amostra conhecidas no passo $v$ também foram utilizados como referência 
para serem comparados com os parâmetros estimados considerando que a distribuição dos probabilidades de seleção da amostra é desconhecida.

No presente trabalho, as implementações computacionais foram realizadas utilizando a linguagem de programação $R$, que foi escolhida por ser um ambiente com código aberto para programação estatística. Devido a ausência de rotinas de otimização nos softwares estatísticos, incluindo a linguagem $R$, a estimação dos parâmetros foi realizada estimando a máxima verissimilhança da distribuição conjunta a posteriori utilizando o pacote de otimização ALGENCAN, cuja descrição pode ser encontrada no Capítulo 3 do presente trabalho.

Depois de estimados os parâmetros, o cálculo do valor de evidência do FBST é realizado em dois passos: otimização numérica e integração numérica. Na implementação da parte de otimização numérica, o pacote ALGENCAN foi novamente utilizado para determinar o ponto de máxima surpresa relativa restrito a hipótese precisa a ser testada. $\mathrm{Na}$ implementação da parte de integração, foi utilizado método de Monte Carlo (descrito no capítulo 3 do presente trabalho) para calcular a integral da distribuição conjunta $a$ posteriori no conjunto de curva de nível definido por esse ponto de máximo.

\subsection{Resultados das simulações}

Começaremos apresentando os resultados obtidos considerando o caso de amostragem aleatória simples, pois este é um desenho amostral não informativo já que as probabilidades de seleção da amostra não estão associadas com a variável resposta. Note que esse método de amostragem está sendo utilizado como referência para avaliar os efeitos da amostragem na estimação dos parâmetros e no valor de evidência.

Desta forma, os resultados computacionais considerando esse tipo de amostragem estão resumidos na Tabela (4.1) onde na primeira coluna estão os valores de cada parâmetro $\beta_{i}$ utilizados para gerar os dados populacionais, nas colunas seguintes estão as médias do viés relativo porcentual dos parâmetros estimados em função de $d$, o grau do polinômio aproximador, e na última coluna estão as médias do viés relativo porcentual dos parâmetros estimados considerando a distribuição das probabilidades de seleção da amostra conhecida. As duas últimas linhas mostram os valores da função $Q Q$ considerando $c=0,95 \mathrm{e}$ 
a razão entre as o número de hipóteses aceitas e o total de casos testados.

Assim, observa-se na Tabela 4.1 que independentemente do valor de $d$ o viés relativo porcentual de todos parâmetros é igual. Isso ocorre porque a amostragem não é informativa e todos os parâmetros $\eta_{j}$ do processo de amostragem foram estimados iguais a zero. Além disso, para todos os valores de $d$ e em todas as amostras consideradas, todas as hipóteses $H_{j}$ foram aceitas. Mostrando assim que a distribuição que melhor ajusta os dados ocorre quando $\eta_{j}=0, \forall j>1$.

\begin{tabular}{cccccccc}
\hline Parâmetros & $\mathbf{d}=\mathbf{0}$ & $\mathbf{d}=\mathbf{1}$ & $\mathbf{d}=\mathbf{2}$ & $\mathbf{d}=\mathbf{3}$ & $\mathbf{d}=\mathbf{4}$ & $\mathbf{d}=\mathbf{5}$ & \multicolumn{1}{c}{$\mathbf{E x p}$} \\
\hline$\beta_{1}=66.60000$ & -0.11608 & -0.11608 & -0.11608 & -0.11608 & -0.11608 & -0.11608 & -0.11608 \\
$\beta_{2}=-10.90000$ & 0.50998 & 0.50998 & 0.50998 & 0.50998 & 0.50998 & 0.50998 & 0.50998 \\
$\beta_{3}=-16.00000$ & -1.25187 & -1.25187 & -1.25187 & -1.25187 & -1.25187 & -1.25187 & -1.25187 \\
$\beta_{4}=-36.50000$ & 1.44637 & 1.44637 & 1.44637 & 1.44637 & 1.44637 & 1.44637 & 1.44637 \\
$\beta_{5}=-7.20000$ & -3.73989 & -3.73989 & -3.73989 & -3.73989 & -3.73989 & -3.73989 & -3.73989 \\
\hline QQ(t,h,0.95) & 0.999613 & 0.999824 & 0.999918 & 0.999961 & 0.999982 & 0.999991 & \\
\hline Razão aceita & 1.00000 & 1.00000 & 1.00000 & 1.00000 & 1.00000 & 1.00000 & \\
\hline
\end{tabular}

Tabela 4.1: Viés relativo porcentual (amostragem aleatória simples com substituição).

As tabelas 4.2-4.6 mostram os resultados das inferências realizadas considerando os métodos de amostragem informativa $i i_{2}-i i i_{6}$ descritos anteriormente. Como já era esperado, quando o modelo populacional $(d=0)$ foi utilizado para estimar os parâmetros,

\begin{tabular}{crrrrrrc}
\hline Parâmetros & \multicolumn{1}{c}{$\mathbf{d}=\mathbf{0}$} & $\mathbf{d}=\mathbf{1}$ & $\mathbf{d}=\mathbf{2}$ & $\mathbf{d}=\mathbf{3}$ & $\mathbf{d}=\mathbf{4}$ & $\mathbf{d}=\mathbf{5}$ & $\mathbf{E x p}$ \\
\hline$\beta_{1}=66.60000$ & -10.40097 & -10.42597 & -0.68316 & -1.19140 & -1.08609 & -0.98790 & 0.39707 \\
$\beta_{2}=-10.90000$ & 1.80049 & 1.62844 & 1.61686 & 1.62741 & 1.62844 & 1.62844 & 1.62844 \\
$\beta_{3}=-16.00000$ & 1.50319 & 1.55504 & 1.56740 & 1.55515 & 1.55504 & 1.55504 & 1.55504 \\
$\beta_{4}=-36.50000$ & 1.59541 & 1.60989 & 1.62720 & 1.60969 & 1.60989 & 1.60989 & 1.60989 \\
$\beta_{5}=-7.20000$ & -3.49992 & -3.81607 & -3.81908 & -3.81716 & -3.81607 & -3.81607 & -3.81607 \\
\hline QQ(t,h,0.95) & 0.999613 & 0.999824 & 0.999918 & 0.999961 & 0.999982 & 0.999991 & \\
\hline Razão aceita & 0.00000 & 0.00000 & 0.79000 & 0.95200 & 0.98400 & 1.00000 & \\
\hline
\end{tabular}

Tabela 4.2: Viés relativo porcentual (amostragem proporcional ao tamanho com substituição). 
o processo de amostragem causou viés principalmente na estimação do parâmetro $\beta_{1}$. Além disso, conforme pode ser observado nessas tabelas, mesmo quando o polinômio aproximador é linear $(d=1)$, o modelo amostral não foi capaz de reduzir significativamente o viés na estimação de $\beta_{1}$ causado pelo processo de amostragem. Observe que nesses dois casos $(d=0$ e $d=1)$, o teste de hipóteses não aceitou nenhuma das estimativas realizadas.

\begin{tabular}{cccccccc}
\hline Parâmetros & $\mathbf{d}=\mathbf{0}$ & $\mathbf{d}=\mathbf{1}$ & $\mathbf{d}=\mathbf{2}$ & $\mathbf{d}=\mathbf{3}$ & $\mathbf{d}=\mathbf{4}$ & $\mathbf{d}=\mathbf{5}$ & $\mathbf{E x p}$ \\
\hline$\beta_{1}=66.60000$ & -9.37323 & -9.37460 & 0.53228 & 0.00227 & 0.01511 & 0.22743 & 1.46454 \\
$\beta_{2}=-10.90000$ & 0.15148 & 0.30077 & 0.30962 & 0.30045 & 0.30077 & 0.30078 & 0.30078 \\
$\beta_{3}=-16.00000$ & 0.41589 & 0.32507 & 0.34308 & 0.32464 & 0.32507 & 0.32507 & 0.32507 \\
$\beta_{4}=-36.50000$ & 2.02514 & 1.93217 & 1.95116 & 1.93218 & 1.93217 & 1.93217 & 1.93217 \\
$\beta_{5}=-7.20000$ & 0.01093 & -0.10439 & -0.19354 & -0.10588 & -0.10440 & -0.10439 & -0.10439 \\
\hline QQ(t,h,0.95) & 0.999613 & 0.999824 & 0.999918 & 0.999961 & 0.999982 & 0.999991 & \\
\hline Razão aceita & 0.00000 & 0.00000 & 0.82000 & 0.95200 & 0.97000 & 1.00000 & \\
\hline
\end{tabular}

Tabela 4.3: Viés relativo porcentual (amostragem sucessiva).

Por outro lado, os resultados obtidos quando a distribuição das probabilidades de seleção da amostra foi aproximada por polinômios quadráticos $(d=2)$ sugerem que modelar a distribuição amostral dessa maneira pode reduzir significativamente o viés causado pelo processo de amostragem. Esta aproximação por um polinômio quadrático foi suficiente para garantir estimativas aceitáveis entre 80,2\% (método de Sampford) e 84\% (amostragem sistemática) dos casos testados.

\begin{tabular}{crrrrrrc}
\hline Parâmetros & $\mathbf{d}=\mathbf{0}$ & $\mathbf{d}=\mathbf{1}$ & $\mathbf{d}=\mathbf{2}$ & $\mathbf{d}=\mathbf{3}$ & $\mathbf{d}=\mathbf{4}$ & $\mathbf{d}=\mathbf{5}$ & $\mathbf{E x p}$ \\
\hline$\beta_{1}=66.60000$ & -9.01824 & -9.00421 & 0.98859 & 0.42229 & 0.49602 & 0.66501 & 1.82972 \\
$\beta_{2}=-10.90000$ & 0.48433 & 0.75401 & 0.76522 & 0.75414 & 0.75401 & 0.75401 & 0.75401 \\
$\beta_{3}=-16.00000$ & 0.24466 & 0.19263 & 0.21127 & 0.19260 & 0.19263 & 0.19263 & 0.19263 \\
$\beta_{4}=-36.50000$ & 2.88782 & 2.73401 & 2.73993 & 2.73407 & 2.73401 & 2.73401 & 2.73401 \\
$\beta_{5}=-7.20000$ & 0.45833 & 1.23387 & 1.23592 & 1.23391 & 1.23387 & 1.23387 & 1.23387 \\
\hline QQ(t,h,0.95) & 0.999613 & 0.999824 & 0.999918 & 0.999961 & 0.999982 & 0.999991 & \\
\hline Razão aceita & 0.00000 & 0.00000 & 0.80200 & 0.96600 & 0.98800 & 1.00000 & \\
\hline
\end{tabular}

Tabela 4.4: Viés relativo porcentual (método de Sampford). 
Além disso, nas amostras em que aproximar as probabilidades de seleção da amostra por um polinômio quadrático $(d=2)$ não foi suficiente para garantir estimativas aceitáveis, o grau desse polinômio aproximador foi aumentado e os resultados das simulações computacionais mostram que polinômios de grau menor ou igual a $d=5$ foram suficientes para garantir estimativas satisfatórias em todos os casos testados.

\begin{tabular}{cccccccc}
\hline Parâmetros & $\mathbf{d}=\mathbf{0}$ & $\mathbf{d}=\mathbf{1}$ & $\mathbf{d}=\mathbf{2}$ & $\mathbf{d}=\mathbf{3}$ & $\mathbf{d}=\mathbf{4}$ & $\mathbf{d}=\mathbf{5}$ & $\mathbf{E x p}$ \\
\hline$\beta_{1}=66.60000$ & -9.51870 & -9.55296 & 0.37016 & -0.18777 & -0.15650 & 0.01484 & 1.25545 \\
$\beta_{2}=-10.90000$ & 0.67584 & 0.37236 & 0.37246 & 0.37403 & 0.37239 & 0.37238 & 0.37523 \\
$\beta_{3}=-16.00000$ & 1.32736 & 1.19228 & 1.20306 & 1.19240 & 1.19228 & 1.19228 & 1.19373 \\
$\beta_{4}=-36.50000$ & 1.81183 & 1.84391 & 1.87106 & 1.84431 & 1.84392 & 1.84391 & 1.84519 \\
$\beta_{5}=-7.20000$ & -5.03366 & -5.52071 & -5.50693 & -5.51950 & -5.52070 & -5.52070 & -5.51647 \\
\hline QQ(t,h,0.95) & 0.999613 & 0.999824 & 0.999918 & 0.999961 & 0.999982 & 0.999991 & \\
\hline Razão aceita & 0.00000 & 0.00000 & 0.82200 & 0.96400 & 0.98000 & 1.00000 & \\
\hline
\end{tabular}

Tabela 4.5: Viés relativo porcentual (amostragem rejeitiva).

Note também que o viés das estimativas obtidas quando a distribuição das probabilidades de seleção da amostra é considerada conhecida (última coluna) está bem próximo do viés obtido aproximando essa distribuição por polinômios e esta distância é menor quando o grau do polinômio aumenta. Isso acontece porque a razão de estimativas aceitas pelo FBST é maior quando o grau do polinômio aumenta e por consequência a média do viés relativo porcentual é calculada considerando um número maior de casos aceitos.

\begin{tabular}{crrrrrrc}
\hline Parâmetros & $\mathbf{d}=\mathbf{0}$ & \multicolumn{1}{c}{$\mathbf{d}=\mathbf{1}$} & $\mathbf{d}=\mathbf{2}$ & $\mathbf{d}=\mathbf{3}$ & $\mathbf{d}=\mathbf{4}$ & $\mathbf{d}=\mathbf{5}$ & $\mathbf{E x p}$ \\
\hline$\beta_{1}=66.60000$ & -10.57869 & -10.61896 & -0.85936 & -1.37865 & -1.32435 & -1.13421 & 0.20835 \\
$\beta_{2}=-10.90000$ & 2.42758 & 2.48535 & 2.46068 & 2.48396 & 2.48536 & 2.48536 & 2.48536 \\
$\beta_{3}=-16.00000$ & 1.90077 & 1.72031 & 1.74190 & 1.72229 & 1.72031 & 1.72031 & 1.72031 \\
$\beta_{4}=-36.50000$ & 1.80781 & 1.74488 & 1.75933 & 1.74969 & 1.74489 & 1.74489 & 1.74489 \\
$\beta_{5}=-7.20000$ & 1.28270 & 0.84182 & 0.79955 & 0.87964 & 0.84182 & 0.84182 & 0.84182 \\
\hline QQ(t,h,0.95) & 0.999613 & 0.999824 & 0.999918 & 0.999961 & 0.999982 & 0.999991 & \\
\hline Razão aceita & 0.00000 & 0.00000 & 0.84000 & 0.96600 & 0.98200 & 1.00000 & \\
\hline
\end{tabular}

Tabela 4.6: Viés relativo porcentual (amostragem sistemática). 
As tabelas 4.7-4.11 mostram resultados que comparam as estimativas dos parâmetros entre os casos aceitos e os casos não-aceitos pelo FBST considerando os valores de $d$ que tiveram casos aceitos e rejeitados. Os casos que tiveram somente casos não-aceitos $(d=0$ e $d=1)$ ou que tiveram somente casos aceitos $(d=5)$ foram desconsiderados. Essas tabelas mostram que as estimativas aceitas pelo teste de hipóteses tem menor viés do que as estimativas não-aceitas.

\begin{tabular}{crrrrrr}
\hline & \multicolumn{2}{c}{$\mathbf{d}=\mathbf{2}$} & \multicolumn{2}{c}{$\mathbf{d}=\mathbf{3}$} & \multicolumn{2}{c}{$\mathbf{d}=\mathbf{4}$} \\
\hline Parâmetros & Aceitos & Rejeitados & Aceitos & Rejeitados & Aceitos & Rejeitados \\
$\beta_{1}=66.60000$ & -0.33055 & -2.00965 & -1.11415 & -2.72348 & -1.02418 & -4.89351 \\
$\beta_{2}=-10.90000$ & 0.38757 & 6.24132 & 1.59908 & 2.18925 & 1.60564 & 3.03067 \\
$\beta_{3}=-16.00000$ & 2.80808 & -3.09994 & 1.95078 & -6.29157 & 1.62301 & -2.62512 \\
$\beta_{4}=-36.50000$ & 2.39355 & -1.25572 & 1.65693 & 0.67283 & 1.75362 & -7.22978 \\
$\beta_{5}=-7.20000$ & -4.19766 & -2.39489 & -4.86633 & 16.99132 & -4.53184 & 40.20375 \\
\hline
\end{tabular}

Tabela 4.7: Viés relativo porcentual: casos aceitos $\times$ casos rejeitados (amostragem proporcional ao tamanho com substituição).

\begin{tabular}{crrrrrr}
\hline & \multicolumn{2}{c}{$\mathbf{d}=\mathbf{2}$} & \multicolumn{2}{c}{$\mathbf{d}=\mathbf{3}$} & \multicolumn{2}{c}{$\mathbf{d}=\mathbf{4}$} \\
\hline Parâmetros & Aceitos & Rejeitados & Aceitos & Rejeitados & Aceitos & Rejeitados \\
$\beta_{1}=66.60000$ & 0.60665 & 0.19350 & 0.08831 & -1.70409 & 0.10658 & -2.94231 \\
$\beta_{2}=-10.90000$ & 0.03690 & 1.55201 & 0.15573 & 3.17081 & -0.23569 & 17.64634 \\
$\beta_{3}=-16.00000$ & 0.90645 & -2.22340 & 0.52857 & -3.72004 & 0.90863 & -18.54334 \\
$\beta_{4}=-36.50000$ & 2.51638 & -0.62371 & 2.07450 & -0.89037 & 2.07001 & -2.52474 \\
$\beta_{5}=-7.20000$ & -1.23249 & 4.53949 & 0.18285 & -5.83221 & 0.45020 & -18.03654 \\
\hline
\end{tabular}

Tabela 4.8: Viés relativo porcentual: casos aceitos $\times$ casos rejeitados (amostragem sucessiva). 


\begin{tabular}{crrrrrr}
\hline & \multicolumn{2}{c}{$\mathbf{d}=\mathbf{2}$} & \multicolumn{2}{c}{$\mathbf{d}=\mathbf{3}$} & \multicolumn{2}{c}{$\mathbf{d}=\mathbf{4}$} \\
\hline Parâmetros & Aceitos & Rejeitados & Aceitos & Rejeitados & Aceitos & Rejeitados \\
$\beta_{1}=66.60000$ & 1.34100 & -0.43884 & 0.61901 & -5.16693 & 0.53978 & -3.10694 \\
$\beta_{2}=-10.90000$ & 2.69240 & -7.04084 & 1.82954 & -29.80011 & 1.52972 & -63.11245 \\
$\beta_{3}=-16.00000$ & 2.02261 & -7.12554 & 0.37087 & -4.87243 & 0.14403 & 4.19429 \\
$\beta_{4}=-36.50000$ & 3.50282 & -0.35015 & 2.85220 & -0.62205 & 2.84660 & -6.53577 \\
$\beta_{5}=-7.20000$ & -0.56643 & 8.53634 & 1.74400 & -13.25866 & 1.37585 & -10.45584 \\
\hline
\end{tabular}

Tabela 4.9: Viés relativo porcentual: casos aceitos $\times$ casos rejeitados (método de Sampford).

\begin{tabular}{crrrrrr}
\hline & \multicolumn{2}{c}{$\mathbf{d}=\mathbf{2}$} & \multicolumn{2}{c}{$\mathbf{d}=\mathbf{3}$} & \multicolumn{2}{c}{$\mathbf{d = 4}$} \\
\hline Parâmetros & Aceitos & Rejeitados & Aceitos & Rejeitados & Aceitos & Rejeitados \\
$\beta_{1}=66.60000$ & 0.92569 & -2.19530 & -0.14013 & -1.46341 & -0.14821 & -0.56267 \\
$\beta_{2}=-10.90000$ & 1.80963 & -6.26439 & 0.42909 & -1.10011 & 0.19015 & 9.30183 \\
$\beta_{3}=-16.00000$ & 2.72461 & -5.82342 & 1.70970 & -12.65965 & 1.47120 & -12.47463 \\
$\beta_{4}=-36.50000$ & 2.67377 & -1.83586 & 1.98234 & -1.85192 & 1.93929 & -2.82934 \\
$\beta_{5}=-7.20000$ & -8.11096 & 6.51839 & -7.06184 & 35.78107 & -5.91750 & 13.92231 \\
\hline
\end{tabular}

Tabela 4.10: Viés relativo porcentual: casos aceitos $\times$ casos rejeitados (amostragem rejeitiva).

\begin{tabular}{crrrrrr}
\hline & \multicolumn{2}{c}{$\mathbf{d}=\mathbf{2}$} & \multicolumn{2}{c}{$\mathbf{d}=\mathbf{3}$} & \multicolumn{2}{c}{$\mathbf{d}=\mathbf{4}$} \\
\hline Parâmetros & Aceitos & Rejeitados & Aceitos & Rejeitados & Aceitos & Rejeitados \\
$\beta_{1}=66.60000$ & -1.14245 & 0.62690 & -1.27715 & -4.26252 & -1.30955 & -2.13153 \\
$\beta_{2}=-10.90000$ & 2.56135 & 1.93219 & 2.66052 & -2.53238 & 2.92722 & -21.62035 \\
$\beta_{3}=-16.00000$ & 1.16081 & 4.79260 & 2.09182 & -8.77687 & 1.96412 & -11.58046 \\
$\beta_{4}=-36.50000$ & 2.10778 & -0.07003 & 1.98123 & -4.82853 & 1.79090 & -0.76540 \\
$\beta_{5}=-7.20000$ & -1.06130 & 10.56898 & 0.29524 & 17.48341 & 0.26485 & 32.31869 \\
\hline
\end{tabular}

Tabela 4.11: Viés relativo porcentual: casos aceitos $\times$ casos rejeitados (amostragem sistemática). 
A Figura 4.1 mostra uma comparação entre as aproximações polinomiais e a distribuição das probabilidades de seleção da amostra. Note que as aproximações polinomiais não sofrem grandes variações dentro do intervalo onde $y_{i}$ assume valores. Além disso, esta figura pode ser utilizada como indicativo para avaliar qual é o grau do polinômio que está mais próximo da função exponencial que está sendo aproximada.

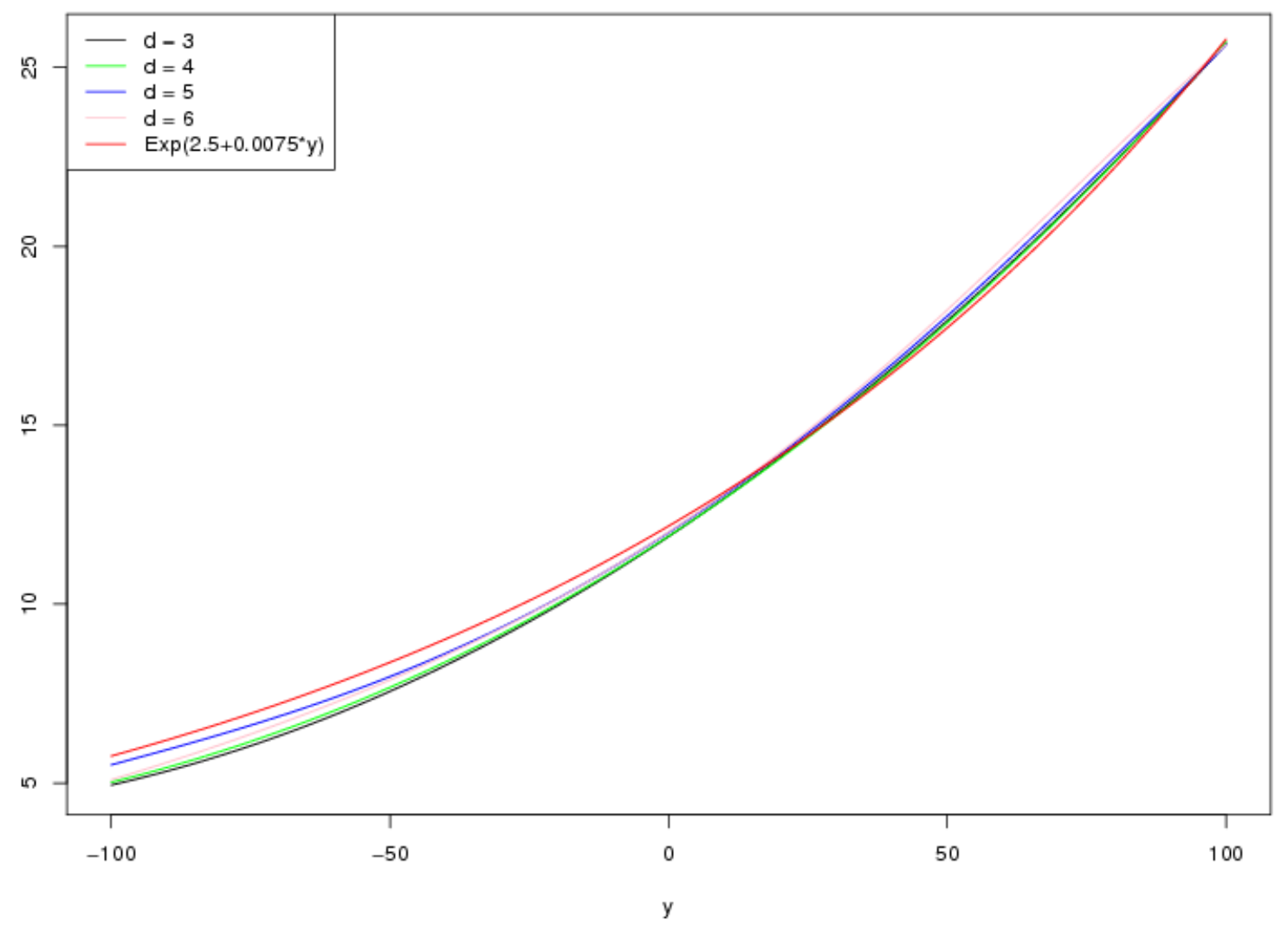

Figura 4.1: Polinômios aproximadores. 


\section{Capítulo 5}

\section{Considerações finais}

No presente trabalho foi utilizada uma distribuição para as medidas amostrais construída a partir da distribuição utilizada para as medidas populacionais e as probabilidades de seleção da amostra de primeira ordem. Utilizar a distribuição amostral como principal vantagem permitir o uso das ferramentas padrão de inferência bayesiana, sem a necessidade de condicionar os valores populacionais às variáveis do processo de amostragem que determinam a probabilidade de seleção.

Foram considerados os casos em que a distribuição das probabilidades de seleção da amostra é conhecida e também os casos em que esta informação não está disponível para quem faz a análise dos dados. Nesses casos de falta de informação, o valor esperado das probabilidades de seleção da amostra foi modelado por um polinômio aproximador de baixo grau dependendo da variável resposta via séries de Taylor. Isso possibilitou que a distribuição amostral da variável resposta pudesse ser definida com os coeficientes do polinômio aproximador incluídos entre os parâmetros do modelo amostral a serem estimados.

Para avaliar a sensibilidade dessas aproximações por polinômios na distribuição conjunta a posteriori foram realizadas simulações computacionais utilizando o software estatístico $R$ e o pacote de otimização ALGENCAN (implementado na linguagem de programação FORTRAN), ambos disponíveis para Linux, MAC OS e Windows. Nessas simulações foram considerados 5 tipos diferentes de amostragem probabilística informativa e tiveram os seguintes objetivos:

i) Avaliar os efeitos práticos de ignorar o processo de seleção da amostra quando o 
mesmo é informativo;

ii) Avaliar a sensibilidade de modelar o valor esperado das probabilidade de seleção da amostra por um polinômio aproximador de baixo grau dependendo da variável resposta e, possivelmente, das variável concomitantes;

iii) Considerando que o modelo populacional é especificado corretamente, avaliar se o processo de seleção da amostra é informativo utilizando o FBST;

iv) Escolher o polinômio de menor grau que ajusta satisfatoriamente os dados quando a amostragem é informativa utilizando o FBST;

v) Comparar o viés entre as estimativas aceitas e as rejeitadas pelo FBST.

Os resultados obtidos mostram que ignorar o processo de amostragem quando as probabilidades de seleção da amostra estão relacionadas com a variável resposta pode causar erros na estimação dos parâmetros, provocando uma análise distorcida do fenômeno estudado. Isso pode ser observado ao comparar o viés das estimativas dos parâmetros utilizando os modelos populacional e amostral no processo de inferência em cada um dos tipos de amostragem considerados.

Além disso, o modelo de inferência utilizando as distribuições amostrais mostrou-se eficiente ao estimar os parâmetros nos casos de amostragem informativa com precisão semelhante ao modelo de inferência padrão para os casos de amostragem não informativa, ocorrendo o mesmo com o valor de evidência.

Bem como mostrou-se também que nos casos de amostragem informativa em que o valor esperado das probabilidades de seleção da amostra foi modelado por um polinômio aproximador com grau suficientemente grande, as estimativas obtidas foram semelhantes às obtidas nos casos em que a distribuição das probabilidades de seleção da amostra é conhecida.

Ademais, considerando que a distribuição populacional é especificada corretamente, as estimativas obtidas para os coeficientes do polinômio aproximador foram utilizadas para avaliar se o processo de amostragem é informativo e os resultados encontrados mostram que o FBST foi eficiente na identificação das amostras selecionadas sob amostragem informativa. 
Com o fim de determinar o polinômio aproximador de menor grau tal que a distribuição conjunta a posteriori ajusta os dados satistoriamente, foi utilizado o valor de evidência do teste de hipóteses FBST considerando o polinômio aproximador de grau $d>0$ na distribuição conjunta a posteriori e definindo a hipótese precisa como o coeficiente do termo de grau $d$ igual a zero. Assim, o teste de hipóteses aceita a hipótese quando o valor desse coeficiente é igual a zero e rejeita caso contrário. Os resultados obtidos mostram que o FBST conseguiu decidir eficientemente o polinômio de menor grau de forma a garantir estimativas com precisão semelhante às encontradas quando a distribuição das probabilidades de seleção da amostra é conhecida. Isso foi feito comparando as estimativas aceitas com as estimativas rejeitadas pelo FBST e observou-se que o viés foi significativamente maior nas estimativas rejeitadas.

Dado o grande potencial prático do presente trabalho, um grande esforço foi dispendido para tornar as implementações computacionais estáveis e robustas numericamente com o objetivo de poderem ser aplicadas a dados reais sem necessidade de grandes ajustes e com bom desempenho computacional.

Por fim, os objetivos iniciais do presente trabalho foram realizados tendo como principais contribuições o desenvolvimento de uma metodologia para avaliar se o processo de amostragem probabilística é informativo e, quando isso ocorre, foram propostas maneira de escolher um modelo que melhor ajuste os dados coletados. Além disso, é importante ressaltar que essa metodologia satisfaz o Princípio da Verossimilhança, permite a incorporação de experiência prévia e a opinião de especialistas, permite a utilização das ferramentas padrão de inferência bayesiana e pode ser facilmente implementada utilizando modernas técnicas de otimização e integração numérica.

Posteriormente, pretende-se implementar os testes de ignorabilidade propostos por Pfeffermann [27] e por Pfeffermann e Sverchkov [29] para comparar com os resultados obtidos no presente trabalho para avaliar se o processo de seleção da amostra é informativo e também para decidir qual o polinômio de menor grau que ajusta os dados com precisão satisfatória utilizando o FBST.

Além disso, o presente trabalho descreve como a definição de distribuição amostral utilizada para fazer inferência baseada em dados oriundos de amostragem probabilística informativa pode ser estendida para casos de não-resposta informativa. Como trabalho futuro, pretende-se ainda utilizar essa metodologia apresentada por Pfeffermann e Sikov 
[33] juntamente com o FBST para avaliar se as probabilidades de não-resposta estão associadas com a variável resposta e dedidir qual modelo melhor se ajusta aos dados. 


\section{Referências Bibliográficas}

[1] R. Andreani, E. G. Birgin, J. M. Martínez e M. L. Schuverdt. On Augmented Lagrangian methods with general lower-level constraints. SIAM Journal on Optimization 18, pags. 1286-1309, 2007.

[2] R. Andreani, E. G. Birgin, J. M. Martínez e M. L. Schuverdt. Augmented Lagrangian methods under the Constant Positive Linear Dependence constraint qualification. Mathematical Programming 111, pags. 5-32, 2008.

[3] J. F. Beaumont. An Estimation Method for Nonignorable Nonresponse. Survey Methodology, vol. 26, número 2, pag. 131-136, 2000.

[4] D. R. Bellhouse. Systematic Sampling. Handbook of Statistics: Sampling, vol. 6, cap. 6, pags. 125-145, Elsevier Science Publishers, 1988.

[5] E. G. Birgin, R. A. Castillo e J. M. Martínez. Numerical comparison of Augmented Lagrangian Algorithms for Nonconvex Problems. Computational Optimization and Applications 31, pags. 31-55, 2005.

[6] W. Borges e J. M. Stern. The rules of logic composition for the Bayesian epistemic e-values. Oxford University Press, 2007.

[7] D. R. Cox e D. V. Hinkley. Theorical statistics. Chapman and Hall: London, 1974.

[8] D. R. Cox. The role of significance tests. Scandinavian Journal of Statistics 4, pag. 49-70, 1977. 
[9] M. Evans e T. Swartz. Approximating integrals via Monte Carlo and deterministic methods. Oxford University Press, 2000.

[10] R . Fletcher. Practical Methods for Optimization, John Wiley and Sons: Chichester, 1987.

[11] A. Gelman, J. Carlin, H. Stern e D. Rubin. Bayesian data analysis, second edition. Chapman and Hall: London, 2004.

[12] J. S. Greenlees, W. S. Reece e K. D. Zieschang. Imputation of Missing Values when the Probability of Response Depends on the Variable being imputed, Journal of the American Statistical Association, vol. 77, número 378, pag. 251-261, 1982.

[13] T. Z. Irony, C. A. B. Pereira e R. C. Tiwari. Analysis of opinion swing: comparison of two correlated proportions. The American Statistician vol. 54(1), pag. 57-62, 2000.

[14] T. Z. Irony, M. Lauretto, C. A. B. Pereira e J. M. Stern. A Weibull wearout test: full Bayesian approach. System Bayesian Reliability, vol. 5, pag. 287-300, 2002.

[15] O. Kempthorne. Of what use are tests of significance and tests of hypothesis. Communications in Statistics - Theory and Methods 8 (A5), pag. 763-777, 1976.

[16] E. L. Lehmann e G. Casella. Theory of Point Estimation. Springer, second edition, 1998.

[17] R. J. A. Little. Models for nonresponse in sample survey. Journal of American Statistics Society 77, pag. 237-250, 1982.

[18] D. G. Luenberger e Y. Ye. Linear and nonlinear programming. Springer, third edition, 2008.

[19] M. R. Madruga, L. G. Esteves e S. Wechsler. On the Bayesinity of Pereira-Stern tests. Sociedad de Estadística e Investigación Operativa 10, pag. 291-299, 2001.

[20] M. R. Madruga, C. A. B. Pereira e J. M. Stern. Bayesian evidence test for precise hipotheses. Journal of Statistical Planning and Inference 117, pag. 185-198, 2003.

[21] Q. McNemar. Note on the Sampling Error of the Difference Between Two Correlated Proportions. Psychometrika 12, pag. 1154-1158, 1947. 
[22] J. Nocedal e S. J. Wright. Numerical Optimization. Springer Verlag: New York, 1999.

[23] C. A. B. Pereira e D. Lindley. Examples questioning the use of partial likelihood. The Statistician 36, pag.15-20, 1987.

[24] C. A. B. Pereira e J. M. Stern. Evidence and credibility: full Bayesian significance test for precise hypotheses. Entropy 1, pag. 69-80, 1999.

[25] C. A. B. Pereira e J. M. Stern. Full Bayesian Significance Tests for Coefficients of Variation. Bayesian Methods with Applications to Science, Polity and Official Statistics, ISBA, pag. 391-400, 2001.

[26] C. A. B. Pereira, J. M. Stern e S. Wechsler. Can a Significance Test Be Genuinely Bayesian? Bayesian Analysis 3(1), pag. 79-100, 2008.

[27] D. Pfeffermann The Role of Sampling Weights when Modeling Survey Data. International Statistics Review 61, pag. 317-337, 1993.

[28] D. Pfeffermann, A. M. Krieger e Y. Rinott. Parametric Distribution of Complex Survey Data under Informative Probability Sampling. Statistica Sinica 8, pag. 1087$1114,1998$.

[29] D. Pfeffermann and M. Sverchkov Parametric and Semi-Parametric Estimation of Regression Models fitted to Survey Data. Sankhya: The Indian Journal of Statistics 61, series B, pag. 166-186, 1999.

[30] D. Pfeffermann e M. Sverchkov. Fitting GLM under informative sampling. Analysis of Survey Data, pag. 175-195. Wiley Series in Survey Methodology, 2003.

[31] D. Pfeffermann, F. A. S. Moura e P. L. N. Silva. Multi-level modelling under informative sampling. Biometrika 93, pag. 943-959, 2006.

[32] D. Pfeffermann e M. Sverchkov. Inference under Informative Sampling. Sample Survey: Inference and Analysis, vol. 29B, pags. 455-465, Elsevier, 2009.

[33] D. Pfeffermann e A. Sikov. Imputation and estimation under nonignorable nonresponse for household surveys with missing covariate information. Journal of Official Statistics, vol. 27 -2 , pags. 181-209, 2011. 
[34] D. B. Rubin. Inference and missing data. Biometrika 63, pag. 581-592, 1976.

[35] D. B. Rubin. Multiple imputations in sample survey - A phenomenological Bayesian approach to nonresponse (with discussion and reply). Imputation and editing of faulty or missing survey data 1-9. U. S. Social Security Administration and Bureau of the Census. 1978.

[36] D. B. Rubin. Conceptual issues in the presence of nonresponse. Nonresponse in sample survey: The theory of current practice, parte III, Panel on incomplete data. Nacional Academy of Sciences, Washington, D. C. 1981.

[37] H. Rubin. A weak system of axioms for "Rational" behaviour and the non-separability of utility from prior. Statist. Decisions 5, pags. 47-58, 1987.

[38] J. M. Stern. Cognitive constructivism and the epistemic significance of sharp statistical hypotheses. 28th International Workshop on Bayesian Inference and Maximum Entropy Methods in Science and Engineering, Boracéia - SP - Brasil, 2008.

[39] J. M. Stern e S. Zacks. Testing the independence of Poisson variables under the Holgate bivariate distribution. Statistics and Probability Letters 60, pag. 313-320, 2002.

[40] R. A. Sudgen e T. M. F. Smith. Ignorable and informative designs in survey sampling inference. Biometrika, vol. 71, pag. 495-506, 1984.

[41] G. Tang, R. J. A. Little e T. E. Raghunathan. Analysis of multivariate missing data with nonignorable nonresponse. Biometrika 90-4, pag. 747-764, 2003.

[42] S. Zacks e J. M. Stern. Sequential estimation of ratios, with application to Bayesian analysis. Tecnical Report RT-MAC-2003-10. 


\section{Índice}

ALGENCAN, 42

amostragem

estratificada, 2

método de Sampford, 52

por conglomerados, 2

proporcional ao tamanho com reposição, 52

rejeitiva, 52

sistemática, 53

sucessiva, 53

conjunto de surpresa relativa mais alta, 37

dados faltantes ao acaso, 18

distribuição

amostral, 4, 9, 21, 22, 27, 49

conjunta a posteriori, 49, 50

populacional, 3, 10, 47

respondente, 4,25

estimação em pequenas áreas, 7

FBST, 33

consistência, 40

definição, 35 função de perda, 38

integração, 43

otimização, 42

função densidade de probabilidade

amostral, 21

função surpresa relativa, 36

função densidade de referência, 36

função densidade de probabilidade

amostral, 22

respondente, 25

hipótese precisa, 34

invariante sob amostragem, 27, 49

mecanismo de não-resposta

ignorável, 4, 19

informativo, 4

modelagem

probabilidade de não-resposta, 25

probabilidade de seleção da amostra, 48

não-resposta ao item, 3

completa, 3

parcial, 3 
parâmetros distintos, 18

pequenas áreas, 7

projeto amostral

estratificado, 30

ignorável, 4, 17

informativo, 4, 15, 48

probabilidade proporcional ao tama-

nho, $6,28,47$

representativo, 1

teste de significância, 8

valor de evidência, 34

verossimilhança

dos dados completos, 17, 19

dos dados observados, 17 


\title{
SURVEY DATA ANALYSIS UNDER INFORMATIVE PROBABILITY SAMPLING
}

\author{
Daniel Mendes Azeredo,**, Julio Michael Stern* \\ Institute of Mathematics and Statistics of the University of São Paulo* \\ dazeredo@ime.usp.br**
}

\begin{abstract}
Pfeffermann, Krieger and Rinott [16] introduced a framework for modeling sampling processes that can be used to assess if a sampling process is informative. In this setting, sample selection probabilities are approximated by a polynomial function depending on outcome and auxiliary variables. Within this framework, our main purpose is to investigate the application of the Full Bayesian Significance Test (FBST), introduced by Pereira and Stern [13], as a tool for testing sampling ignorability, that is, to detect a significant relation between the sample selection probabilities and the outcome variable. The performance of this statistical modelling framework is tested with some simulation experiments.
\end{abstract}

Keywords: Design variables; Informative sampling; Sample distribution; PPS sampling; Sampling ignorability; FBST - Full Bayesian Significance Test

PACS: 02.50.Tt, 07.05.Fb, 07.05.Kf.

\section{INTRODUCTION}

Survey data are routinely used in studies related to economics, agriculture, education, politics, health and several other scientific fields. The information collected in this kind of research is essential for economic, social and urban planning, development of medical and educational procedures, improvement of public services, etc. The main objective of collecting survey data is to estimate characteristics of the general population under study using information from a given sample.

The sampling process is informative if the sample selection probabilities are related to the values of the model response variable even after conditioning on auxiliary variables, as more carefully defined in Section 2. In such a situation, the selection effects need to be considered in the inference process.

An interesting methodology to deal with informative sampling was introduced by Pfeffermann, Krieger and Rinott [16] in which the authors define an expression to compute a sample distribution as a function of the population distribution and sample selection probabilities. When this methodology is used, estimation of the pertinent distributions only requires information about units selected on the sampling process, that is, it does not require auxiliary variables to be observed for units of the population that were not selected.

Using standard conjugate distributions, the resulting sample distributions have simple analytical form, allowing straightforward implementation of common inference procedures like maximum likelihood estimation, least square methods, residual analysis or Bayesian analysis. Furthermore, the conjugacy mechanism used in this process is the 
very same used for conjugate priors in Bayesian models, a mechanism already familiar to most researchers in statistics, see Cox and Hindley [7]. Finally, the sample selection probabilities can be approximated by a polynomial function depending both on outcome and auxiliary variables. We propose using robust test statistics based on the Full Bayesian Significance Test (FBST) for deciding if the relation between this probabilities and the outcome variable is significant and for choosing the degree of the best fitting polynomial function.

Section 2 defines the expression used to calculate the sample distribution according to Pfeffermann, Krieger and Rinott [16] and presents a theorem stating conditions that ensure general invariance relations for the functional form of the population and sample distributions. Section 3 presents a review of the tests of sampling ignorability and also a robust alternative based on the Full Bayesian Significance Test. Section 4 explains the computational tools used to perform the simulation experiment. Section 5 describes a case study, performing and analyzing some simulation experiments. Finally, Section 6 presents our final remarks.

\section{SAMPLE PROBABILITY DENSITY FUNCTION}

We consider $U=\{1,2, \ldots, N\}$ and $A_{U}=\left\{\left(y_{1}, x_{1}, z_{1}\right), \ldots,\left(y_{N}, x_{N}, z_{N}\right)\right\}$ a finite population defined by $N$ realizations of a stochastic process $(Y, X, Z)$, where vector $Y$ has the outcome variables of the statistical model, matrix $X$ has the covariate variables, and matrix $Z$ is a matrix of design variables used for sample selection. Typically, the covariate matrix $X$ includes auxiliary variables like strata indicators, age, gender, income or education level and others measurements known only after sampling. The design matrix $Z_{U}=\left(z_{1}, \ldots, z_{N}\right)$ may (stochastically) depend on some or all the covariates $X$ and, in some cases, also the outcome variables $Y$. Moreover, matrix $Z$ is assumed to be known by a surveyor, who designs the sampling process, but not necessarily known by the analyst, who receives the survey data and is in charge of statistical inference.

We also consider the indicator variable $I=\left(I_{1}, I_{2}, \ldots, I_{N}\right)$ such that $I_{i}=1$ if the unit $i$ of the population is selected for the sample, otherwise $I_{i}=0$. Hence, the subset $S=\left\{i \in U \mid I_{i}=1\right\}$ characterizes the selected sample of population $U$. The sample selection probabilities are defined as $\pi_{i}=\operatorname{Pr}\left(I_{i}=1 \mid Z_{U}\right)$, and we will consider only sampling processes where $\pi_{i}>0, \forall i \in U$. We assume these probabilities $\pi_{1}, \ldots, \pi_{N}$ to be known by the surveyor who designs the sampling process, and they are also known (or must be modeled) by the data analyst who performs the statistical inference, at least for the sampled units.

We assume that the population distribution of the outcome variable $Y_{i}$ depends on known values of concomitant variables $x_{i}, Y_{i} \sim f_{p}\left(y_{i} \mid x_{i}, \theta\right)$, where $\theta$ is the parameter vector of the statistical model. Thus, following the methodology introduced by Pfeffermann, Krieger and Rinott [16], the sample distribution of $Y_{i}$ in the case of full response is defined as

$$
f_{s}\left(y_{i} \mid x_{i}\right)=f\left(y_{i} \mid x_{i}, I_{i}=1\right)=\operatorname{Pr}\left(I_{i}=1 \mid y_{i}, x_{i}\right) f_{p}\left(y_{i} \mid x_{i}\right) / \operatorname{Pr}\left(I_{i}=1 \mid x_{i}\right) .
$$

Consequently, the population and the sample distributions are equal only when 
$\operatorname{Pr}\left(I_{i}=1 \mid y_{i}, x_{i}\right)=\operatorname{Pr}\left(I_{i}=1 \mid x_{i}\right)$ for all $y_{i}$. According to Rubin [19], under this condition the sampling process is called ignorable. Otherwise, the sampling process is called informative. Note that the parameters of the population distribution are included among the parameters of sample distribution and these parameters may be estimated by maximum likelihood or some other standard method.

The probabilities $\operatorname{Pr}\left(I_{i}=1 \mid y_{i}, x_{i}\right)$ are generally different from the sample inclusion probabilities $\pi_{i}=\operatorname{Pr}\left(I_{i}=1 \mid Z_{U}\right)$, which depends on all the population values of $Z$. Regarding these probabilities $\pi_{i}$ as random variables, the following relation holds

$$
\operatorname{Pr}\left(I_{i}=1 \mid y_{i}, x_{i}\right)=\int \operatorname{Pr}\left(I_{i}=1 \mid y_{i}, x_{i}, \pi_{i}\right) f_{p}\left(\pi_{i} \mid y_{i}, x_{i}\right) d \pi_{i}=E_{p}\left(\pi_{i} \mid y_{i}, x_{i}\right),
$$

for $\operatorname{Pr}\left(I_{i}=1 \mid y_{i}, x_{i}, \pi_{i}\right)=\pi_{i}$. Substituting (2) into (1), an alternative expression for the sample distribution of $Y_{i}$ in the case of full response is

$$
f_{s}\left(y_{i} \mid x_{i}\right)=E_{p}\left(\pi_{i} \mid y_{i}, x_{i}\right) f_{p}\left(y_{i} \mid x_{i}\right) / E_{p}\left(\pi_{i} \mid x_{i}\right)
$$

An important characteristic of the expression (3) is that, given the population distribution, the sample distribution is easily calculated when $E_{p}\left(\pi_{i} \mid y_{i}, x_{i}\right)$ is known, as a consequence of $E_{p}\left(\pi_{i} \mid x_{i}\right)=\int E_{p}\left(\pi_{i} \mid y_{i}, x_{i}\right) f_{p}\left(y_{i} \mid x_{i}\right) d y_{i}$. Furthermore, when the hypothesized form of the population distribution and the expectation $E_{p}\left(\pi_{i} \mid y_{i}, x_{i}\right)$ are given, the form of the sample distribution is uniquely defined by the expression (3).

Notice that the Pfeffermann's methodology makes use of auxiliary variables for units selected on the sampling process, but does not require auxiliary variables to be observed for non-selected units of the population. Moreover, under informative sampling, the sample complement, $\tilde{S}=U-S$, is also an informative sampling, but the model fitting the units in $\tilde{S}$ is different from the model fitting the units in $S$, and also different from the model fitting the entire population. Under appropriate conditions, the difference between the models fitting the entire population and the sample units will be small as the ratio between the sample size increases.

Using equation (1), Pfeffermann, Krieger and Rinott [16] show the following invariance conditions ensuring that population and sample distribution have the same analytical form.

Proposition 1 Consider a population distribution in the exponential family,

$$
f_{p}\left(y_{i} \mid x_{i}\right)=a_{i}\left(\theta_{i}\right) \exp \left[\sum_{k=1}^{P} \theta_{k i} b_{k i}\left(y_{i}\right)+c_{i}\left(y_{i}\right)\right],
$$

where $\theta_{i}=\left(\theta_{1 i}, \theta_{2 i}, \ldots, \theta_{P i}\right)$ defines the natural parameterization of the family taking values in the parameter space $\Theta$, and $b_{k i}$ and $c_{i}$ are known functions. Furthermore, consider that the sample selection probabilities have expectations

$$
E_{p}\left(\pi_{i} \mid y_{i}, x_{i}\right)=r_{i} \exp \left[\sum_{k=1}^{P} \psi_{k i} b_{k i}\left(y_{i}\right)\right]
$$


where $r_{i}$ are constants that may depends on $x_{i}$ but not on $y_{i}$ and $\psi_{k i}$ are the parameters used in sample design. Then the sample distribution of $y_{i}$ belongs to the same exponential family with parameters $\theta_{k i}^{*}=\theta_{k i}+\psi_{k i}$, provided $\theta_{k i}^{*} \in \Theta$.

The dependence on $x_{i}$ in the equations (4) and (5) operates via the parameters $\theta_{k i}$ and $\psi_{k i}$ respectively. In the special case of a linear relation,

$$
\theta_{k i}=\phi_{0 k}+x_{i}^{T} \phi_{k} \text { and } \psi_{k i}=\gamma_{0 k}+x_{i}^{T} \gamma_{k}
$$

this dependence on $x_{i}$ may be made more explicit for a class of regression models of $Y$ on $X$.

Corollary 1 Under the conditions of Proposition 1 and linear relation (6), the sample distribution belongs to same exponential family with parameters

$$
\theta_{i k}^{*}=\left(\phi_{0 k}+\gamma_{0 k}\right)+x_{i}^{T}\left(\phi_{k}+\gamma_{k}\right) \text {. }
$$

In particular, if the functions $\psi_{k i}$ do not depend on $x_{i}$, that is, if $\gamma_{k}=0$, then the coefficients of $x_{i}$ in the natural parameterization of the sample distribution are the same as the one for the population distribution.

Example 1 (Linear regression model) Consider that the population model fitting the outcome variable $Y$ is

$$
f_{p}\left(y_{i} \mid x_{i}\right)=N\left(x_{i}^{T} \beta, \sigma^{2}\right), i=1, \ldots, N
$$

where $\beta=\left(\beta_{1}, \ldots, \beta_{p}\right) \in \mathbb{R}^{p}$ and $\sigma^{2}$ are the parameters to be estimated and $X$ is a data matrix $n \times p$ such that the first column is equal to 1 .

Moreover, suppose that the sampling is carried out with probability proportional to size where sizes $z_{i}$ satisfy the log-normal distribution

$$
f_{p}\left(\log \left(z_{i}\right) \mid y_{i}, x_{i}\right)=N\left(\gamma_{1}+\gamma_{2} y_{i}, \sigma_{z}^{2}\right)
$$

where $\gamma_{1}, \gamma_{2}$ and $\sigma_{z}$ are unknown parameters to be estimated. This model is clearly informative when $\gamma_{2} \neq 0$, since the size $z_{i}$ used in the sample selection process depends on the outcome variable.

Note that, under probability proportional to size, $\pi_{i}=\operatorname{Pr}\left(I_{i}=1 \mid Z_{U}\right)=C z_{i}$, where $C=n /(N \bar{z})$ is constant and $\bar{z}=\frac{1}{N} \sum_{i=1}^{N} z_{i}$ is the population mean of the sizes $z$.

Hence, using the properties of log-normal distribution

$$
E_{p}\left[\pi_{i} \mid y_{i}, x_{i}\right]=C \exp \left[\gamma_{1}+\gamma_{2} y_{i}+\frac{\sigma_{z}^{2}}{2}\right]
$$

Consequently, according to Proposition 1, the sample distribution of $y_{i} \mid x_{i}$ is

$$
f_{s}\left(y_{i} \mid x_{i}\right)=N\left(x_{i}^{T} \beta+\gamma_{2} \sigma^{2}, \sigma^{2}\right) .
$$


On the other hand, since the sample distribution (11) depends on $\gamma_{2}$ and considering $z_{i}$ known only in the sampled units, the sample distribution of $\log \left(z_{i}\right) \mid y_{i}$ should be included in the inference process. Accordingly, since $E_{p}\left(\pi_{i} \mid z_{i}, y_{i}\right)=C z_{i}$, it follows that

$$
E_{p}\left(\pi_{i} \mid y_{i}\right)=C \exp \left[\gamma_{1}+\gamma_{2} y_{i}+\frac{\sigma_{z}^{2}}{2}\right] .
$$

Consequently, using again the Proposition 1, the sample distribution of $\log \left(z_{i}\right) \mid y_{i}$ is

$$
f_{s}\left(\log \left(z_{i}\right) \mid y_{i}\right)=N\left(\gamma_{1}+\gamma_{2} y_{i}+\sigma_{z}^{2}, \sigma_{z}^{2}\right) .
$$

Note that the regression of $Y$ on $X$ in the sample distribution is the same as in the population distribution except for the intercept term $\beta_{1}$ which changes for $\beta_{1}+\gamma_{2} \sigma^{2}$. The same happens in the regression of $\log (Z)$ on $Y$ where the intercept term $\gamma_{1}$ in population distribution changes for $\gamma_{1}+\sigma_{z}^{2}$ in sample distribution.

The conjugacy mechanism used in Proposition 1 and its Corollaries works using exacly the same integration operations used in the prior-posterior distribution update operations of Bayesian learning. Cox and Hindley [7] call a family of prior distributions for which the posterior distribution will be in the same family as - closed under sampling, a very appropriate name in the present context. Castilho, Cobo and Gutierrez [5], Castilho, Iglessias and Ruiz-Cobo [6], Diaconis and Ylvisaker [8], Fink [10], George, Makov and Smith [11], and Stern [21] give a larger perspective on how functional invariance properties play an essential role in Bayesian statistics.

\section{Probability proportional to size sampling}

As previously stated, we assume that the sample selection probabilities, $\pi_{1}, \ldots, \pi_{N}$, are known (or must be modeled) by the analyst in charge of statistical inference, at least for the sampled units. Hence, the expectation of $\pi_{i} \mid y_{i}, x_{i}$ could be used to study if the sampling process is informative. Moreover, since in practical sampling the sample selection probabilities distribution is generally unknown by the analyst, it is reasonable to approximate the expectation of $\pi_{i} \mid y_{i}, x_{i}$ by a Taylor series expansion by a polynomial function in $y_{i}$ and $x_{i}=\left(x_{i 1}, x_{i 2}, \ldots, x_{i m}\right)$, having the form

$$
\begin{aligned}
& E_{p}\left(\pi_{i} \mid y_{i}, x_{i}\right) \approx \sum_{j=0}^{d} \eta_{j} y_{i}^{j}+\sum_{p=1}^{m} h\left(x_{i p}\right), \\
& h\left(x_{i p}\right)=\sum_{k=1}^{l(p)} \lambda_{k p} x_{i p}^{k}=\lambda_{1 p} x_{i p}+\lambda_{2 p} x_{i p}^{2}+\lambda_{3 p} x_{i p}^{3}+\cdots+\lambda_{l(p) p} x_{i p}^{l(p)}
\end{aligned}
$$

where $d$ is the degree of the polynomial in $y_{i}, h$ is a polynomial function in $x_{i p}$ of degree $l(p)$, and $\left\{\eta_{j}\right\}$ and $\left\{\lambda_{i p}^{k}\right\}$ are the unknown parameters to be estimated. 
Furthermore, assuming that the moments $E_{p}\left(y_{i}^{j} \mid x_{i}\right)$ exist,

$$
E_{p}\left(\pi_{i} \mid x_{i}\right) \approx \eta_{0}+\sum_{j=1}^{d} \eta_{j} E_{p}\left(y_{i}^{j} \mid x_{i}\right)+\sum_{p=1}^{m} h\left(x_{i p}\right)
$$

Hence, the sample distribution of $y_{i} \mid x_{i}$ can be written as

$$
f_{s}\left(y_{i} \mid x_{i}\right)=\frac{E_{p}\left(\pi_{i} \mid y_{i}, x_{i}\right) f_{p}\left(y_{i} \mid x_{i}\right)}{E_{p}\left(\pi_{i} \mid x_{i}\right)}=\frac{\left[\sum_{j=0}^{d} \eta_{j} y_{i}^{j}+\sum_{p=1}^{m} h\left(x_{i p}\right)\right] f_{p}\left(y_{i} \mid x_{i}\right)}{\eta_{0}+\sum_{j=1}^{d} \eta_{j} E_{p}\left(y_{i}^{j} \mid x_{i}\right)+\sum_{p=1}^{m} h\left(x_{i p}\right)} .
$$

On the other hand, since $\pi_{i}=\operatorname{Pr}\left(I_{i}=1 \mid Z_{U}\right)=C z_{i}$ with $C=n /(N \bar{z})$ constant, the expected values of $\pi_{i}$ and the sample distribution of $z_{i} \mid y_{i}, x_{i}$ are given by

$$
\begin{aligned}
& E_{p}\left(\pi_{i} \mid z_{i}, y_{i}, x_{i}\right)=C E_{p}\left(z_{i} \mid z_{i}, y_{i}, x_{i}\right)=C z_{i}, \\
& E_{p}\left(\pi_{i} \mid y_{i}, x_{i}\right)=C \int z_{i} f_{p}\left(z_{i} \mid y_{i}, x_{i}\right) d z_{i}=C\left[\sum_{j=0}^{d} \eta_{j} y_{i}^{j}+\sum_{p=1}^{m} h\left(x_{i p}\right)\right], \\
& f_{s}\left(z_{i} \mid y_{i}, x_{i}\right)=\frac{E_{p}\left(\pi_{i} \mid z_{i}, y_{i}, x_{i}\right) f_{p}\left(z_{i} \mid y_{i}, x_{i}\right)}{E_{p}\left(\pi_{i} \mid y_{i}, x_{i}\right)}=\frac{z_{i} f_{p}\left(z_{i} \mid y_{i}, x_{i}\right)}{\sum_{j=0}^{d} \eta_{j} y_{i}^{j}+\sum_{p=1}^{m} h\left(x_{i p}\right)} .
\end{aligned}
$$

Moreover, considering the design parameters $\psi=\left(\eta_{0}, \ldots, \eta_{d}, \lambda_{11}, \ldots, \lambda_{l(m) m}\right)$, the population parameters $\theta$, and the prior density $f(\theta, \psi)$, the joint posterior distribution is

$$
f(\theta, \psi \mid y, X, z) \propto f(\theta, \psi) \prod_{i=1}^{n} f_{s}\left(y_{i} \mid x_{i}\right) \prod_{i=1}^{n} f_{s}\left(z_{i} \mid y_{i}, x_{i}\right),
$$

where $f_{s}\left(y_{i} \mid x_{i}\right)$ is defined in (17) and $f_{s}\left(z_{i} \mid y_{i}, x_{i}\right)$ is defined in (20).

\section{TESTING SAMPLING IGNORABILITY}

In complex sampling designs, a direct check of Rubin [19] sampling ignorability conditions may be difficult and are often impractical. Nevertheless, whether or not to treat a sampling as ignorable is a practical question that the analyst must decide in order to proceed to statistical inference. In this section we examine some statistical tests used to decide if a sampling process is ignorable or informative.

Several of such test procedures may be found in the literature. Most of them are based on Linear Regression analysis, testing the significance of the difference between the estimators found when the sampling process is ignored (population model) and the estimators obtained using another particular model. We should remark that all ignorability 
tests under consideration depend on a correct specification of the statistical model for the population.

For example, Pfeffermann [15] tests ignorability conditions when sampling weights are used in the inference process by considering the null hypothesis $H_{0}: \operatorname{plim}_{n \rightarrow \infty} N \rightarrow \infty\left(\hat{\beta}-\hat{\beta}_{w}\right)=0$, where $\hat{\beta}$ is the ordinary least square estimator of $\beta, \hat{\beta}_{w}$ is the probability-weighted estimator of $\beta$ and plim means the limit in probability under randomization distribution. Pfeffermann [15] focus on the test statistic

$$
\lambda=\hat{D}^{T}[\hat{V}(\hat{D})]^{-1} \hat{D},
$$

where $\hat{D}=\hat{\beta}-\hat{\beta}_{w}$ is the difference between the aforementioned estimators and $\hat{V}(\hat{D})$ is an estimator of the variance-covariance matrix of $\hat{D}$. Note that the estimator $\hat{\beta}_{w}$ is consistent for $\beta$ when the ordinary least square estimator fits the population model, that is $\operatorname{plim}_{n \rightarrow \infty} N \rightarrow \infty \hat{\beta}_{w}=\beta$. Furthermore, the estimator $\hat{\beta}$ is consistent for $\beta$ under ignorable sampling, but $\hat{\beta}$ is no longer consistent for $\beta$ if the sampling process is informative, when the two estimators converge to different limits.

DuMouchel and Duncan [9] propose testing the ignorability of a sampling process for estimating the parameter $\beta$ by augmenting the matrix $X$ of concomitants variables of the population model by the columns $Z=W X$, where $W=\operatorname{diag}\left(w_{1}, w_{2}, \ldots, w_{n}\right)$, and fitting the regression model $Y=X \beta+Z \eta$ using ordinary least squares. They show that to test a null hypothesis $H_{0}$ representing ignorability is equivalent to test the hypothesis $H_{0}^{*}: \eta=0$.

Pfeffermann and Sverchkov [17] propose yet another way to test ignorability considering the residual term $\varepsilon_{i}=y_{i}-E_{p}\left(y_{i} \mid x_{i}\right)$ in each unit $i$ and testing if $E_{p}\left(\varepsilon_{i}^{k}\right)=E_{s}\left(\varepsilon_{i}^{k}\right)$, for $i=1, \ldots, n$ and $k>0$. This is equivalent to set hypotheses $\operatorname{Corr}_{s}\left(\varepsilon_{i}^{k}, w_{i}\right)=0$, where Corr $_{s}$ is the correlation under the sample distribution, $w_{i}=1 / \pi_{i}$ and $k>0$. According to Pfeffermann and Sverchkov [17], in practice it suffices to test the first 2 or 3 correlations.

Considering the polynomial approximation (14) and the joint posterior distribution (21), one can test sampling ignorability testing if the polynomial function is constant. For this purpose, one can study the polynomial degree $d$ that best fits the data. If $\eta_{j} \neq 0$ for some $j \geq 1$ then the sampling process is informative and the joint posterior distribution (21) should be used in the inference process. A possible way to decide the degree $d$ of the best fitting polynomial is to use the Full Bayesian Significance Test (FBST) to test the hypotheses $H_{d}: \eta_{k}=0, \forall k>d$. This FBST based metodology for model selection is explained in great detail at Pereira and Stern [14].

The decision of accepting or rejecting hypothesis $H_{d}$ is based on ev $(H)$, the $e$-value or evidence value supporting the hypothesis, according to a pre-established confidence level threshold given by the function

$$
Q Q(t, h, c)=Q\left(t-h, Q^{-1}(t, c)\right), \text { where } Q(k, x)=\frac{\Gamma(k / 2, x / 2)}{\Gamma(k / 2, \infty)},
$$

$t$ is the parameter space dimension, $h$ is the hypothesis dimension and $Q(k, x)$ is the cumulative chi-square distribution with $k$ degrees of freedom. For more details, see Borges and Stern [4]. 


\section{COMPUTATIONAL ASPECTS}

The computational simulations carried out in the next section concern the example of Pfeffermann's methodology for handling informative sampling described in previous sections, including the polynomial function model for sampling probabilities. More specifically, we will focus on the performance of the FBST as a tool for model selection. Our computational experiments were implemented using language $\mathrm{R}$, an open source environment for statistical computing. In our R environment, the standard available functionalities were supplemented by the high performance optimization package Algencan, developed by the Tango (Trustable Algorithms for Nonlinear General Optimization) project at USP and UNICAMP. This package implements advanced Augmented Lagrangian optimization methods and provides friendly interfaces for users of R, AMPL, C/C++, CUTEr, Java, Matlab, Octave, Python, TCL, and other popular programming environments. More details about Algencan can be found in Andreani, Birgin and Martinez [1] and Andreani, Birgin and Martinez [2]. With the help of Algencan, we could easily implement the numerical optimization step of the FBST, that is, to find the maximizing argument, $\left(\theta^{\star}, \psi^{\star}\right)$ and the maximum value, $f^{\star}$, of the joint posterior distribution under the constraints imposed by $H_{d}$.

The numerical integration step uses a MCMC (Markov Chain Monte Carlo) method for computing the ratio

$$
e v\left(H_{d}, y, X, z\right)=\frac{\int g(\theta, \psi \mid y, X, z) h_{1}(\theta) h_{2}(\psi) d \theta d \psi}{\int f(\theta, \psi \mid y, X, z) h_{1}(\theta) h_{2}(\psi) d \theta d \psi}
$$

where $g(\theta, \psi \mid y, X, z)=I\left(f(\theta, \psi \mid y, X, z)>f^{\star}\right) f(\theta, \psi \mid y, X, z)$, and $h_{1}(\theta)$ and $h_{2}(\psi)$ are the importance sampling densities used at the MCMC, see Lauretto et al. [12].

\section{CASE STUDY}

Our case study is based on Pfeffermann, Moura and Silva [18]. The population model fitting the response variable $Y$ is normally distributed, with concomitant variables $X=\left[x_{1}, \ldots x_{N}\right]^{T}$ such that

$$
f_{p}\left(y_{i} \mid x_{i}\right)=N\left(x_{i}^{T} \beta, \sigma^{2}\right), i=1, \ldots, N,
$$

where $\beta=\left(\beta_{1}, \ldots, \beta_{p}\right) \in \mathbb{R}^{p}$ is the parameter to be estimated, $X$ is a data matrix $N \times p$ having as the first column the unit vector 1 , and the variance $\sigma^{2}=693$ is known. The values of $\beta$ (listed in the tables below) and $\sigma^{2}$ were also chosen according to Pfeffermann, Moura and Silva [18].

We suppose that $n$ units of the population are sampled with sampling proportional to size, with log-normally distributed $z_{i}$,

$$
f_{p}\left(\log \left(z_{i}\right) \mid y_{i}, x_{i}\right)=N\left(\gamma_{1}+\gamma_{2} y_{i}, \sigma_{z}^{2}\right),
$$

where $\gamma_{1}$ and $\gamma_{2}$ are unknown parameters and the variance $\sigma_{z}^{2}=c^{2}=1$ is known. The fitted values $\gamma_{1}=2.5$ and $\gamma_{2}=0.0075$ were also chosen according to the simulations 
presented in Pfeffermann, Moura and Silva [18]. According to condition (26), the sampling process is clearly informative when $\gamma_{2} \neq 0$, since the size $z_{i}$ used in the sample selection process depends on the response variable $y_{i}$.

We consider two cases for modeling expectations of inclusion in the sampling process. In the first case, the distribution of sample inclusion is known by the analyst. In the second case, the analyst uses a Taylor series approximation, that is, a low order polynomial function on $y_{i}$.

When the distribution of the sample inclusion probabilities is known in the inference process, according to Example 1, the sample distribution of $y_{i} \mid x_{i}$ is

$$
f_{s}\left(y_{i} \mid x_{i}\right)=N\left(x_{i}^{T} \beta+\gamma_{2} \sigma^{2}, \sigma^{2}\right),
$$

and the sample distribution of $\log \left(z_{i}\right) \mid y_{i}$ is

$$
f_{s}\left(\log \left(z_{i}\right) \mid y_{i}\right)=N\left(\gamma_{1}+\gamma_{2} y_{i}+\sigma_{z}^{2}, \sigma_{z}^{2}\right) .
$$

Considering the non-informative prior density $f\left(\beta, \gamma_{1}, \gamma_{2}\right)=1$, the joint posterior distribution can be written as

$f\left(\beta, \gamma_{1}, \gamma_{2} \mid y, X, z, \sigma^{2}, \sigma_{z}^{2}\right) \propto$

$$
\begin{aligned}
& \propto \prod_{i=1}^{n}\left[f_{s}\left(\log \left(z_{i}\right) \mid y_{i}\right) f_{s}\left(y_{i} \mid x_{i}\right)\right] \\
& \propto \prod_{i=1}^{n}\left[N\left(\gamma_{1}+\gamma_{2} y_{i}+\sigma_{z}^{2} \mid \log \left(z_{i}\right), \sigma_{z}^{2}\right) N\left(x_{i}^{T} \beta+\gamma_{2} \sigma^{2} \mid y_{i}, \sigma^{2}\right)\right]
\end{aligned}
$$

and the $\log$ of joint posterior distribution as

$$
\begin{aligned}
& \text { lf }\left(\beta, \gamma_{1}, \gamma_{2} \mid y, X, z, \sigma^{2}, \sigma_{z}^{2}\right) \propto \\
& \quad \propto \sum_{i=1}^{n} \log \left[N\left(\gamma_{1}+\gamma_{2} y_{i}+\sigma_{z}^{2} \mid \log \left(z_{i}\right), \sigma_{z}^{2}\right)\right]+\sum_{i=1}^{n} \log \left[N\left(x_{i}^{T} \beta+\gamma_{2} \sigma^{2} \mid y_{i}, \sigma^{2}\right)\right] .
\end{aligned}
$$

Hence, the partial derivates are

$$
\begin{aligned}
\frac{\partial}{\partial \beta_{k}} l f\left(\beta, \gamma_{1}, \gamma_{2} \mid y, X, z, \sigma^{2}, \sigma_{z}^{2}\right) & \propto \frac{1}{\sigma^{2}} \sum_{i=1}^{n}\left(y_{i}-x_{i}^{T} \beta-\gamma_{2} \sigma^{2}\right) x_{i k} \\
\frac{\partial}{\partial \gamma_{1}} l f\left(\beta, \gamma_{1}, \gamma_{2} \mid y, X, z, \sigma^{2}, \sigma_{z}^{2}\right) & \propto \frac{1}{\sigma_{z}^{2}} \sum_{i=1}^{n}\left(\log \left(z_{i}\right)-\gamma_{1}-\gamma_{2} y_{i}-\sigma_{z}^{2}\right) \\
\frac{\partial}{\partial \gamma_{2}} l f\left(\beta, \gamma_{1}, \gamma_{2} \mid y, X, z, \sigma^{2}, \sigma_{z}^{2}\right) & \propto \frac{1}{\sigma_{z}^{2}} \sum_{i=1}^{n}\left[\left(\log \left(z_{i}\right)-\gamma_{1}-\gamma_{2} y_{i}-\sigma_{z}^{2}\right) y_{i}\right] \\
& +\sum_{i=1}^{n}\left(y_{i}-x_{i}^{T} \beta-\gamma_{2} \sigma^{2}\right) .
\end{aligned}
$$


When, on the other hand, the polynomial approximation is used,

$$
E_{p}\left(z_{i} \mid y_{i}\right)=\eta_{0}+\eta_{1} y_{i}+\eta_{2} y_{i}^{2}+\cdots+\eta_{d} y_{i}^{d}, \text { for some } d \geq 0 \text {. }
$$

Using the expression (21) and considering the noninformative prior distribution $f\left(\beta, \eta_{0}, \ldots, \eta_{d}\right)=1$, the joint posterior distribution is

$$
f\left(\beta, \eta_{0}, \ldots, \eta_{d} \mid y, X, z, \sigma^{2}, \sigma_{z}^{2}\right) \propto \prod_{i=1}^{n} \frac{z_{i} N\left(x_{i}^{T} \beta \mid y_{i}, \sigma^{2}\right) N\left(\sum_{j=0}^{d} \eta_{j} y_{i}^{j} \mid z_{i}, \sigma_{z}^{2}\right)}{\eta_{0}+\sum_{j=1}^{d} \eta_{j} E_{p}\left(y_{i}^{j} \mid x_{i}\right)} .
$$

Hence, the log of joint posterior distribution can be written as

$$
\begin{aligned}
l f\left(\beta, \eta_{0}, \ldots, \eta_{d} \mid y, X, z, \sigma^{2}, \sigma_{z}^{2}\right) & \propto \sum_{i=1}^{n} \log \left(z_{i}\right)+\sum_{i=1}^{n} \log \left[N\left(\sum_{j=0}^{d} \eta_{j} y_{i}^{j} \mid z_{i}, \sigma_{z}^{2}\right)\right] \\
& +\sum_{i=1}^{n} \log \left[N\left(x_{i}^{T} \beta \mid y_{i}, \sigma^{2}\right)\right] \\
& -\sum_{i=1}^{n} \log \left[\sum_{j=0}^{d} \eta_{j} E_{p}\left(y_{i}^{j} \mid x_{i}\right)\right] .
\end{aligned}
$$

Consequently, the partial derivatives required to estimate the maximum likelihood are

$$
\begin{aligned}
& \frac{\partial}{\partial \beta_{k}} l f\left(\beta, \eta_{0}, \ldots, \eta_{d} \mid y, X, z, \sigma^{2}, \sigma_{z}^{2}\right) \propto \\
& \quad \propto \frac{1}{\sigma^{2}}(y-X \beta)^{T} x_{\cdot k}-\sum_{i=1}^{n} \frac{\left[\sum_{j=0}^{d} \eta_{j} \frac{\partial}{\partial \beta_{k}} E_{p}\left(y_{i}^{j} \mid x_{i}\right)\right]}{\left[\sum_{j=0}^{d} \eta_{j} E_{p}\left(y_{i}^{j} \mid x_{i}\right)\right]},
\end{aligned}
$$

and

$$
\begin{aligned}
\frac{\partial}{\partial \eta_{k}} & l f\left(\beta, \eta_{0}, \ldots, \eta_{d} \mid y, X, z, \sigma^{2}, \sigma_{z}^{2}\right) \propto \\
& \propto \frac{1}{\sigma_{z}^{2}} \sum_{i=1}^{n} y_{i}^{k}\left(z_{i}-\sum_{j=0}^{d} \eta_{j} y_{i}^{j}\right)-\sum_{i=1}^{n} \frac{E_{p}\left(y_{i}^{k} \mid x_{i}\right)}{\left[\sum_{j=0}^{d} \eta_{j} E_{p}\left(y_{i}^{j} \mid x_{i}\right)\right]} .
\end{aligned}
$$

Our simulation experiment was conducted according to the following procedure, always based on the aforementioned models and the case study of Pfeffermann, Moura and Silva [18].

i) Generate $N=500$ independent population measurements $\left\{Y_{1}, Y_{2}, \ldots, Y_{N}\right\}$ from the distribution $f_{p}\left(y_{i} \mid x_{i}\right)$ given in (25) with the values of $\beta$ listed in the tables below and the $x$-values generated from $\operatorname{Gamma}\left(\alpha_{x}, \beta_{x}\right)$, where $\alpha_{x}$ and $\beta_{x}$ were drawn at random with uniform distribution on the interval $[0,1]$.

ii) Generate population measurements for a design variable $Z_{i}$ according to the expression (26). 
iii) For each population drawn, select with probability proportional to size (PPS) one sample with $n=50$ elements using $Z$ as the size variable following the following method of sampling:

iii 1 ) Simple random sampling without replacement.

iii2) PPS sampling with replacement.

iii 3 ) Successive sampling: Draw one unit each time with replacement with probabilities proportional to $P_{k}=Z_{k} /(N \bar{Z})$ until $n$ distinct units have been selected.

iii $)$ Sampford's method: The first unit of sample is selected with probability $P_{k}$ and the remaining $(n-1)$ units are selected with probabilities proportional to $P_{i} /\left(1-n P_{i}\right)$ with replacement. The sample is accepted if the $n$ units drawn are all distinct, otherwise it is rejected and the process is repeated (see more details in Sampford [20]).

iii 5 ) Rejective sampling: One unit of sample is selected each time with replacement with probabilities proportional to $\rho_{k}=P_{k} /\left(1-P_{k}\right)$ and the sample is reject if a unit selected at previous draw is choose again. The process is repeated until $\mathrm{t}$ completion of a sample where all units are distinct.

iii $\left.{ }_{6}\right)$ Systematic PPS sampling: Considering $c=n / N$, a random start $r$ between 1 and $c$ is selected. Then every $c$-th unit thereafter $r, r+c, r+2 c, \ldots, r+(n-1) c$ is selected (see more details in Bellhouse [3]).

iv) Perform the statistical analysis considering that the distribution of the size $z_{i}$ is unknown, that is, using the joint posterior distribution defined at (34). The polynomial degree $d$ is the lowest so that the e-value of hypothesis $H_{d}$ becomes greater than $Q Q(t, h, 0.95)$.

v) Perform the statistical analysis considering that the distribution of the size $z_{i}$ is known, that is, using the joint posterior distribution defined at (29).

Note that the sampling method $\mathrm{iii}_{1}$ is not informative and was used as a benchmark for assessing the sampling effects on the inference process. On the other hand, the sampling methods $\mathrm{iii}_{2}-i_{i} i_{6}$ are informative under the condition (26). In the same way, the parameters estimated in the case of known sample inclusion distributions, in step $v$, were used as a benchmark for comparison with the case of unknown sample inclusion distribution.

The results of our simulation study are summarized in the following tables 1-6. The first column contains the numerical value of each parameter $\beta_{i}$; the next columns contain percentual relative mean biases as a function of the polynomial degree $d$; and the last column contains percentual relative mean biases when the distribution of sample selection probabilities $z_{i}$ is known. The last two rows shows the value of $Q Q$ function considering $c=0.95$ and the acceptance ratio for the hypothesis being tested.

Table 1 displays the results of the inference process when the simple random sampling without replacement is used. In this case, the sampling scheme is not informative. Hence, as expected, the estimated parameters for any degree of the approximating polynomial function is the same, all estimated parameters $\eta_{j}$, with $j>0$, are equal to zero, and all the hypotheses $H_{d}$ were accepted. 
TABLE 1. Percentual Relative Biases (Simple random sampling without replacement).

\begin{tabular}{ccccrrrr}
\hline Parameters & $\mathbf{d}=\mathbf{0}$ & $\mathbf{d}=\mathbf{1}$ & $\mathbf{d}=\mathbf{2}$ & $\mathbf{d = 3}$ & $\mathbf{d = 4}$ & $\mathbf{d = 5}$ & \multicolumn{1}{c}{$\operatorname{Exp}$} \\
\hline$\beta_{1}=66.60000$ & -0.11608 & -0.11608 & -0.11608 & -0.11608 & -0.11608 & -0.11608 & -0.11608 \\
$\beta_{2}=-10.90000$ & 0.50998 & 0.50998 & 0.50998 & 0.50998 & 0.50998 & 0.50998 & 0.50998 \\
$\beta_{3}=-16.00000$ & -1.25187 & -1.25187 & -1.25187 & -1.25187 & -1.25187 & -1.25187 & -1.25187 \\
$\beta_{4}=-36.50000$ & 1.44637 & 1.44637 & 1.44637 & 1.44637 & 1.44637 & 1.44637 & 1.44637 \\
$\beta_{5}=-7.20000$ & -3.73989 & -3.73989 & -3.73989 & -3.73989 & -3.73989 & -3.73989 & -3.73989 \\
\hline QQ(t,h,0.95) & 0.999613 & 0.999827 & 0.999918 & 0.99996 & 0.999982 & 0.999991 & \\
\hline Accept.ratio & 1.00000 & 1.00000 & 1.00000 & 1.00000 & 1.00000 & 1.00000 & \\
\hline
\end{tabular}

Tables 2-6 display the results of the inference process considering the informative sampling schemes $i i_{2}-i i_{6}$. As expected, truncating too early the polynomial approximation, say using polynomials of degree $d=0$ or $d=1$, produces strongly biased estimators for some parameters, for example $\beta_{1}$. On the other hand, the results found when the distribution of the sample selection probabilities was approximated by a polynomial function with degree $d=2$ show a significant reduction on the same bias. This approximation was enough to ensure fair acceptance ratios in all cases at hand, from $80.2 \%$ for Sampford's method to $84 \%$ for systematic sampling. Even better acceptance ratios and smaller bias where obtained with higher order polynomial functions, displayed at the tables

TABLE 2. Percentual Relative Biases (PPS sampling with replacement).

\begin{tabular}{crrrrrrr}
\hline Parameters & $\mathbf{d = 0}$ & $\mathbf{d = 1}$ & $\mathbf{d = 2}$ & $\mathbf{d = 3}$ & $\mathbf{d = 4}$ & $\mathbf{d = 5}$ & Exp \\
\hline$\beta_{1}=66.60000$ & -10.40097 & -10.42597 & -0.68316 & -1.19140 & -1.08609 & -0.98790 & 0.39707 \\
$\beta_{2}=-10.90000$ & 1.80049 & 1.62844 & 1.61686 & 1.62741 & 1.62844 & 1.62844 & 1.62844 \\
$\beta_{3}=-16.00000$ & 1.50319 & 1.55504 & 1.56740 & 1.55515 & 1.55504 & 1.55504 & 1.55504 \\
$\beta_{4}=-36.50000$ & 1.59541 & 1.60989 & 1.62720 & 1.60969 & 1.60989 & 1.60989 & 1.60989 \\
$\beta_{5}=-7.20000$ & -3.49992 & -3.81607 & -3.81908 & -3.81716 & -3.81607 & -3.81607 & -3.81607 \\
\hline QQ(t,h,0.95) & 0.999613 & 0.999824 & 0.999918 & 0.999961 & 0.999982 & 0.999991 & \\
\hline Accept.ratio & 0.00000 & 0.00000 & 0.79000 & 0.95200 & 0.98400 & 1.00000 & \\
\hline
\end{tabular}

TABLE 3. Percentual Relative Biases (Successive sampling).

\begin{tabular}{cccccccc}
\hline Parameters & $\mathbf{d = 0}$ & $\mathbf{d = 1}$ & $\mathbf{d = 2}$ & $\mathbf{d = 3}$ & $\mathbf{d = 4}$ & $\mathbf{d = 5}$ & Exp \\
\hline$\beta_{1}=66.60000$ & -9.37323 & -9.37460 & 0.53228 & 0.00227 & 0.01511 & 0.22743 & 1.46454 \\
$\beta_{2}=-10.90000$ & 0.15148 & 0.30077 & 0.30962 & 0.30045 & 0.30077 & 0.30078 & 0.30078 \\
$\beta_{3}=-16.00000$ & 0.41589 & 0.32507 & 0.34308 & 0.32464 & 0.32507 & 0.32507 & 0.32507 \\
$\beta_{4}=-36.50000$ & 2.02514 & 1.93217 & 1.95116 & 1.93218 & 1.93217 & 1.93217 & 1.93217 \\
$\beta_{5}=-7.20000$ & 0.01093 & -0.10439 & -0.19354 & -0.10588 & -0.10440 & -0.10439 & -0.10439 \\
\hline QQ(t,h,0.95) & 0.999613 & 0.999824 & 0.999918 & 0.999961 & 0.999982 & 0.999991 & \\
\hline Accept.ratio & 0.00000 & 0.00000 & 0.82000 & 0.95200 & 0.97000 & 1.00000 & \\
\hline
\end{tabular}


1-6 up to degree $d=5$. Furthermore, at least for the case study at hand, a confidence level of $95 \%$ seems to provide a good cutoff threshold for choosing the degree of the Taylor series expansion.

TABLE 4. Percentual Relative Biases (Sampford's method).

\begin{tabular}{cccccccc}
\hline Parameters & $\mathbf{d = 0}$ & $\mathbf{d = 1}$ & $\mathbf{d = 2}$ & $\mathbf{d = 3}$ & $\mathbf{d = 4}$ & $\mathbf{d = 5}$ & $\mathbf{E x p}$ \\
\hline$\beta_{1}=66.60000$ & -9.01824 & -9.00421 & 0.98859 & 0.42229 & 0.49602 & 0.66501 & 1.82972 \\
$\beta_{2}=-10.90000$ & 0.48433 & 0.75401 & 0.76522 & 0.75414 & 0.75401 & 0.75401 & 0.75401 \\
$\beta_{3}=-16.00000$ & 0.24466 & 0.19263 & 0.21127 & 0.19260 & 0.19263 & 0.19263 & 0.19263 \\
$\beta_{4}=-36.50000$ & 2.88782 & 2.73401 & 2.73993 & 2.73407 & 2.73401 & 2.73401 & 2.73401 \\
$\beta_{5}=-7.20000$ & 0.45833 & 1.23387 & 1.23592 & 1.23391 & 1.23387 & 1.23387 & 1.23387 \\
\hline QQ(t,h,0.95) & 0.999613 & 0.999824 & 0.999918 & 0.999961 & 0.999982 & 0.999991 & \\
\hline Accept.ratio & 0.00000 & 0.00000 & 0.80200 & 0.96600 & 0.98800 & 1.00000 & \\
\hline
\end{tabular}

TABLE 5. Percentual Relative Biases (Rejective sampling).

\begin{tabular}{cccccrcc}
\hline Parameters & $\mathbf{d = 0}$ & $\mathbf{d = 1}$ & $\mathbf{d = 2}$ & $\mathbf{d = 3}$ & $\mathbf{d = 4}$ & $\mathbf{d = 5}$ & $\operatorname{Exp}$ \\
\hline$\beta_{1}=66.60000$ & -9.51870 & -9.55296 & 0.37016 & -0.18777 & -0.15650 & 0.01484 & 1.25545 \\
$\beta_{2}=-10.90000$ & 0.67584 & 0.37236 & 0.37246 & 0.37403 & 0.37239 & 0.37238 & 0.37523 \\
$\beta_{3}=-16.00000$ & 1.32736 & 1.19228 & 1.20306 & 1.19240 & 1.19228 & 1.19228 & 1.19373 \\
$\beta_{4}=-36.50000$ & 1.81183 & 1.84391 & 1.87106 & 1.84431 & 1.84392 & 1.84391 & 1.84519 \\
$\beta_{5}=-7.20000$ & -5.03366 & -5.52071 & -5.50693 & -5.51950 & -5.52070 & -5.52070 & -5.51647 \\
\hline QQ(t,h,0.95) & 0.999613 & 0.999824 & 0.999918 & 0.999961 & 0.999982 & 0.999991 & \\
\hline Accept.ratio & 0.00000 & 0.00000 & 0.82200 & 0.96400 & 0.98000 & 1.00000 & \\
\hline
\end{tabular}

TABLE 6. Percentual Relative Biases (Systematic sampling).

\begin{tabular}{crrcrrrc}
\hline Parameters & $\mathbf{d = 0}$ & $\mathbf{d = 1}$ & $\mathbf{d = 2}$ & $\mathbf{d = 3}$ & $\mathbf{d = 4}$ & $\mathbf{d = 5}$ & $\mathbf{E x p}$ \\
\hline$\beta_{1}=66.60000$ & -10.57869 & -10.61896 & -0.85936 & -1.37865 & -1.32435 & -1.13421 & 0.20835 \\
$\beta_{2}=-10.90000$ & 2.42758 & 2.48535 & 2.46068 & 2.48396 & 2.48536 & 2.48536 & 2.48536 \\
$\beta_{3}=-16.00000$ & 1.90077 & 1.72031 & 1.74190 & 1.72229 & 1.72031 & 1.72031 & 1.72031 \\
$\beta_{4}=-36.50000$ & 1.80781 & 1.74488 & 1.75933 & 1.74969 & 1.74489 & 1.74489 & 1.74489 \\
$\beta_{5}=-7.20000$ & 1.28270 & 0.84182 & 0.79955 & 0.87964 & 0.84182 & 0.84182 & 0.84182 \\
\hline QQ(t,h,0.95) & 0.999613 & 0.999824 & 0.999918 & 0.999961 & 0.999982 & 0.999991 & \\
\hline Accept.ratio & 0.00000 & 0.00000 & 0.84000 & 0.96600 & 0.98200 & 1.00000 & \\
\hline
\end{tabular}

Tables 7-11 compare the average quality of the estimated parameters when the FBST accepts of rejects hypothesis $H_{d}$. In these tables, the first column contains the numerical values of the parameters $\beta_{i}$, and the following columns contain the mean percentual relative biases of their estimates as a function of the polynomial degree $d$, divided in two distinct groups, according to acceptance or rejection by the FBST at the $95 \%$ level. We do not tabulate the cases $d=0, d=1$ and $d=5$ for they produce full rejection, for $d=0$ and $d=1$, or full acceptance, for $d=5$. 
TABLE 7. Percentual Relative Biases (PPS sampling with replacement).

\begin{tabular}{crrrrrr}
\hline & \multicolumn{2}{c}{$\mathbf{d = 2}$} & \multicolumn{2}{c}{$\mathbf{d = 3}$} & \multicolumn{2}{c}{$\mathbf{d = 4}$} \\
\hline Parameters & Accepted & Rejected & Accepted & Rejected & Accepted & Rejected \\
$\beta_{1}=66.60000$ & -0.33055 & -2.00965 & -1.11415 & -2.72348 & -1.02418 & -4.89351 \\
$\beta_{2}=-10.90000$ & 0.38757 & 6.24132 & 1.59908 & 2.18925 & 1.60564 & 3.03067 \\
$\beta_{3}=-16.00000$ & 2.80808 & -3.09994 & 1.95078 & -6.29157 & 1.62301 & -2.62512 \\
$\beta_{4}=-36.50000$ & 2.39355 & -1.25572 & 1.65693 & 0.67283 & 1.75362 & -7.22978 \\
$\beta_{5}=-7.20000$ & -4.19766 & -2.39489 & -4.86633 & 16.99132 & -4.53184 & 40.20375 \\
\hline
\end{tabular}

TABLE 8. Percentual Relative Biases (Successive sampling).

\begin{tabular}{crrrrrr}
\hline & \multicolumn{2}{c}{$\mathbf{d = 2}$} & \multicolumn{2}{c}{$\mathbf{d = 3}$} & \multicolumn{2}{c}{$\mathbf{d = 4}$} \\
\hline Parameters & Accepted & Rejected & Accepted & Rejected & Accepted & Rejected \\
$\beta_{1}=66.60000$ & 0.60665 & 0.19350 & 0.08831 & -1.70409 & 0.10658 & -2.94231 \\
$\beta_{2}=-10.90000$ & 0.03690 & 1.55201 & 0.15573 & 3.17081 & -0.23569 & 17.64634 \\
$\beta_{3}=-16.00000$ & 0.90645 & -2.22340 & 0.52857 & -3.72004 & 0.90863 & -18.54334 \\
$\beta_{4}=-36.50000$ & 2.51638 & -0.62371 & 2.07450 & -0.89037 & 2.07001 & -2.52474 \\
$\beta_{5}=-7.20000$ & -1.23249 & 4.53949 & 0.18285 & -5.83221 & 0.45020 & -18.03654 \\
\hline
\end{tabular}

TABLE 9. Percentual Relative Biases (Sampford's method).

\begin{tabular}{crrrrrr}
\hline & \multicolumn{2}{c}{$\mathbf{d = 2}$} & \multicolumn{2}{c}{$\mathbf{d = 3}$} & \multicolumn{2}{c}{$\mathbf{d = 4}$} \\
\hline Parameters & Accepted & Rejected & Accepted & Rejected & Accepted & Rejected \\
$\beta_{1}=66.60000$ & 1.34100 & -0.43884 & 0.61901 & -5.16693 & 0.53978 & -3.10694 \\
$\beta_{2}=-10.90000$ & 2.69240 & -7.04084 & 1.82954 & -29.80011 & 1.52972 & -63.11245 \\
$\beta_{3}=-16.00000$ & 2.02261 & -7.12554 & 0.37087 & -4.87243 & 0.14403 & 4.19429 \\
$\beta_{4}=-36.50000$ & 3.50282 & -0.35015 & 2.85220 & -0.62205 & 2.84660 & -6.53577 \\
$\beta_{5}=-7.20000$ & -0.56643 & 8.53634 & 1.74400 & -13.25866 & 1.37585 & -10.45584 \\
\hline
\end{tabular}

TABLE 10. Percentual Relative Biases (Rejective sampling).

\begin{tabular}{crrrrrr}
\hline & \multicolumn{2}{c}{$\mathbf{d = 2}$} & \multicolumn{2}{c}{$\mathbf{d = 3}$} & \multicolumn{2}{c}{$\mathbf{d = 4}$} \\
\hline Parameters & Accepted & Rejected & Accepted & Rejected & Accepted & Rejected \\
$\beta_{1}=66.60000$ & 0.92569 & -2.19530 & -0.14013 & -1.46341 & -0.14821 & -0.56267 \\
$\beta_{2}=-10.90000$ & 1.80963 & -6.26439 & 0.42909 & -1.10011 & 0.19015 & 9.30183 \\
$\beta_{3}=-16.00000$ & 2.72461 & -5.82342 & 1.70970 & -12.65965 & 1.47120 & -12.47463 \\
$\beta_{4}=-36.50000$ & 2.67377 & -1.83586 & 1.98234 & -1.85192 & 1.93929 & -2.82934 \\
$\beta_{5}=-7.20000$ & -8.11096 & 6.51839 & -7.06184 & 35.78107 & -5.91750 & 13.92231 \\
\hline
\end{tabular}

Figure 1 shows a graphical comparison between some Taylor series expansions and the targeted exponential function. This figure can be used for choosing, by visual inspection, the best degree for the polynomial approximation, and compare this choice with our numerical selection procedures. 
TABLE 11. Percentual Relative Biases (Systematic sampling).

\begin{tabular}{crrrrrr}
\hline & \multicolumn{2}{c}{$\mathbf{d = 2}$} & \multicolumn{2}{c}{$\mathbf{d = 3}$} & \multicolumn{2}{c}{$\mathbf{d = 4}$} \\
\hline Parameters & Accepted & Rejected & Accepted & Rejected & Accepted & Rejected \\
$\beta_{1}=66.60000$ & -1.14245 & 0.62690 & -1.27715 & -4.26252 & -1.30955 & -2.13153 \\
$\beta_{2}=-10.90000$ & 2.56135 & 1.93219 & 2.66052 & -2.53238 & 2.92722 & -21.62035 \\
$\beta_{3}=-16.00000$ & 1.16081 & 4.79260 & 2.09182 & -8.77687 & 1.96412 & -11.58046 \\
$\beta_{4}=-36.50000$ & 2.10778 & -0.07003 & 1.98123 & -4.82853 & 1.79090 & -0.76540 \\
$\beta_{5}=-7.20000$ & -1.06130 & 10.56898 & 0.29524 & 17.48341 & 0.26485 & 32.31869 \\
\hline
\end{tabular}

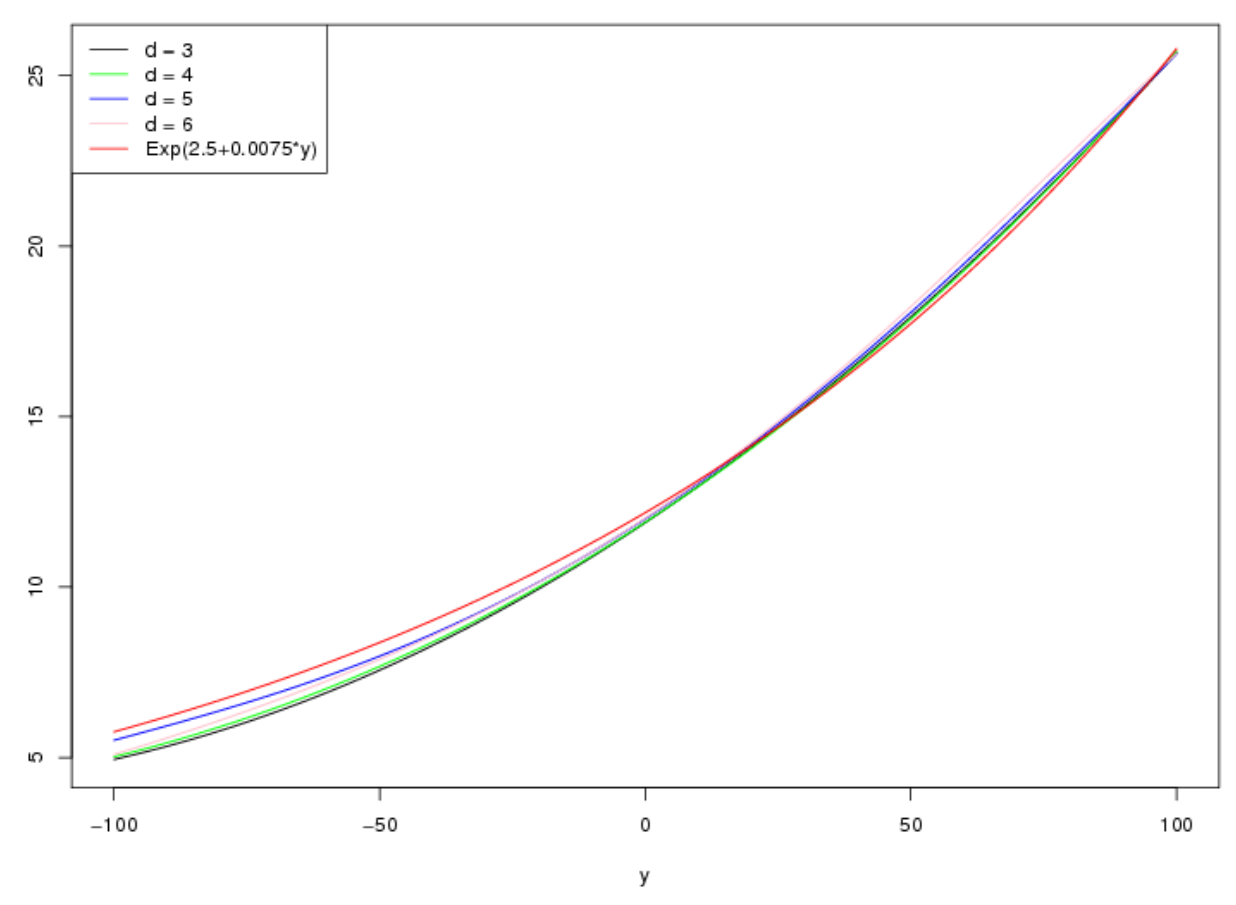

FIGURE 1. Approximating polynomials and target function.

\section{FINAL REMARKS}

In this paper we review how the methodology introduced by Pfeffermann, Krieger and Rinott [16] can be used to perform statistical inference from survey data obtained under informative sampling. In this methodology, sample selection probabilities are approximated by a Taylor series expansion, implying a critical decision concerning the optimal degree of the approximating polynomial function. We showed how the FBST, the Full Bayesian Significance Test, can be used for this task, using the FBST significance measure, the $e$-value, as the core statistic in a model selection procedure. Some simulation experiments, based on a case study of Pfeffermann, Moura and Silva [18], seem to indicate that this selection mechanism is stable and reliable, possibly 
extending the range of applications of both methodologies.

\section{ACKNOWLEDGMENTS}

The authors are grateful for the support received at IME-USP, The Institute of Mathematics and Statistics of the University of São Paulo and also for the support received from the following agencies: CNPq, The Brazilian National Council for Scientific and Technological Research, and FAPESP, The São Paulo State Research Foundation.

\section{REFERENCES}

1. Andreani R., Birgin E. G., Martinez J. M. and Schuverdt M. L. On Augmented Lagrangian methods with general lower-level constraints. SIAM Journal on Optimization 18, pp. 1286-1309, 2007.

2. Andreani R., Birgin E. G., Martinez J. M. and Schuverdt M. L. Augmented Lagrangian methods under the Constant Positive Linear Dependence constraint qualification. Mathematical Programming 111, pp. 5-32, 2008.

3. Bellhouse D. R. Systematic Sampling. Handbook of Statistics: Sampling, vol. 6, chapter 6, pp. 125$145,1988$.

4. Borges W. and Stern J. M. The Rules of Logic Composition for the Bayesian Epistemic e-Values. Oxford University Press: Logic Journal of the IGPL 15, pp. 401-420, 2007.

5. Castillo E., Cobo A., Gutierrez J. A. and Pruneda R. E. Functional Networks with Applications: A Neural-Based Paradigm. NY: Springer, 1998.

6. Castillo E., Iglessias A. and Ruiz-Cobo R. Functional Equations in Applied Sciences (Sec. 13.8 Bayesian Conjugate Distributions). Amsterdam: Elsevier, 2004.

7. Cox D. R. and Hinkley D. V. Theoretical Statistics, chapter 10. Chapman and Hall. London, 1974.

8. Diaconis P. and Ylvisaker D. Conjugate priors for exponential families. Annals of Statistics 7, pp. 269-281, 1979.

9. DuMouchel W. H. and Duncan G. J. Using Sample Survey Weights in Multiple Regression Analysis of Stratified Samples. Journal of American Statistical Association 78, pp. 535-543, 1983.

10. Fink D. A Compendium of Conjugate Priors. Technical report, Cornell University, 1995

11. George E. I., Makov U. E. and Smith A. F. M. Conjugate Likelihood Distributions. Scandinavian Journal of Statistics 20, 2, pp. 147-156, 1993.

12. Lauretto M., Pereira C.A.B., Stern J.M. and Zacks S. Full Bayesian Signicance Test Applied to Multivariate Normal Structure Models. Brazilian Journal of Probability and Statistics, 17, pp. 147168, 2003.

13. Pereira C. A. B. and Stern J. M. Evidence and Credibility: full Bayesian significance test for precise hypotheses. Entropy 1, pp. 69-80, 1999.

14. Pereira C. A. B. and Stern J. M. Model Selection and Regularization: Full Bayesian Approach. EnvironMetrics, 12, 6, pp. 559-568, 2001.

15. Pfeffermann D. The Role of Sampling Weights when Modeling Survey Data. International Statistics Review 61, pp. 317-337, 1993.

16. Pfeffermann D., Krieger A. M. and Rinott Y. Parametric distribution of complex survey data under Informative Probability Sampling. Statistica Sinica 8, pp. 1087-1114, 1998.

17. Pfeffermann D. and Sverchkov M. Parametric and Semi-Parametric Estimation of Regression Models fitted to Survey Data. Sankhya: The Indian Journal of Statistics 61, series B, pp. 166-186, 1999.

18. Pfeffermann D., Moura F. A. S. and Silva P. L. N. Multi-level modelling under Informative Sampling. Biometrika 93, pp. 943-959, 2006.

19. Rubin D. B. Inference and Missing Data. Biometrika 63, pp. 581-592, 1976.

20. Sampford, M. R. On sampling without replacement with unequal probabilities of selection. Biometrika 54, pp. 499-513, 1967.

21. Stern J. M. Symmetry, Invariance and Ontology in Physics and Statistics. Symmetry 3, pp. 611-635, 2011. 